\title{
Performance Studies and Energy Management of Aggregated BESSs for Frequency Regulation
}

\author{
Zhang Tian
}

School of Electrical and Electronic Engineering

A thesis submitted to Nanyang Technological University in partial fulfillment of the requirements for the degree of Doctor of Philosophy (EEE) 

Dedicated to my parents, who opened my door to the pursuit of an intellectual life. 



\section{Acknowledegment}

First and foremost, I am honored to pursue my $\mathrm{PhD}$ degree under the guidance of Associate Professor Gooi Hoay Beng, who has been very diligent, knowledgeable, insightful, and always in pursuit of high quality works and standards. His proactive attitude in work and life greatly inspires me during my study.

I shall also extend my great gratitude to my colleagues and fellow researchers, including Dr. Chen Shuaixun, Dr. Wang Mingqiang, Mr. Dante Fernando Recalde Melo, and Miss Faranak Golestaneh for benevolently sharing their knowledge and engaging in technical discussions. I would also like to thank our technical staff Mrs. Chia-Nge Tak Heng, Mr. Thomas Foo and Miss Lin Zhiren in Clean Energy Research Laboratory, for their continuous support and facilitation of accessing our resources.

My special gratitude goes to my family and all my friends, especially Ali Al-Sheikh and Ursula Del Carmen Romero, who have always been wise, encouraging and extremely supportive. These inspiring and passionate people ignite beautiful sparks in life, adding up to the great memories for this period of my life.

Last but not the least, I would like to thank Nanyang Technological University for rewarding the research scholarship and providing an international and stimulating learning environment. 



\section{Abstract}

With the increasing installed capacity of renewable energy sources (RESs) in the power systems, their uncertainty and intermittency are causing power imbalances in the grid, which leads to frequency regulation issues. When rapid fluctuations take place, the system requires fast responding regulation resources to recover the frequency within a short period of time. While traditional power plants with slow dynamics are less capable of tracking the fast-changing regulation signals, battery energy storage systems (BESSs) are considered as an effective regulation source to respond immediately to frequency deviations.

From the system operator's point of view, a proper procedure for BESS sizing and control is required to ensure a long-term high quality regulation service on the system level. We study the sizing issue of a BESS aggregation based on the overall system control performance. The frequency performance with different penetration rates of BESS is analyzed. The frequency performance is evaluated by Control Performance Standard 1 (CPS1) and Control Performance Standard 2 (CPS2) proposed by North American Electricity Reliability Corporation (NERC). The recommended BESS penetration rate in the power system is analyzed using an iterative approach according to the NERC performance indices. Taking the investment cost into consideration, a 5\% to $15 \%$ BESS penetration rate is recommended for the test system.

For a network penetrated with dispersedly located RESs, distributed energy resources (DERs) and storage devices, the virtual power plant (VPP) concept is applied to aggregate the resources. A centralized hierarchical controller is proposed for the VPP to achieve a more economical operation and more effective system frequency regulation. Model predictive control (MPC) strategy is implemented in the controller. The VPP's influence on system frequency is studied by real-time simulations. The results show that the secondary control executes economic dispatch to coordinate the power dispatch within the VPP according to photovoltaic (PV) power output, load data, and real-time electricity tariff. In the meantime, the primary controller is capable of stabilizing sys- 
tem frequency within the permitted range during heavy load and peak solar generation periods.

The proposed hierarchical control scheme is further developed for a distribution network with distributed BESSs which can be aggregated as a VPP. As it is difficult for BESSs to be profitable due to high battery costs, the aggregated BESSs are maneuvered to participate in multiple markets. We propose a hierarchical energy management system (HiEMS) for aggregated BESSs taking part in both energy and regulation markets. The HiEMS performs optimal scheduling in multiple markets and attempts to coordinate BESSs of different battery types, various state of charge (SOC), and power and energy capacity. The SOC values are regulated around the expected average SOC to prevent individual saturation or depletion, and thus increasing the average battery lifetime. The proposed HiEMS can support up to 0.5 regulation participation rate, which will boost the cost performance index (CPI) by 7 times. HiEMS outperforms participation factor (PF) method by 1.24 times and master-slave (M-S) method by 1.4. In terms of time of first replacement, HiEMS is 2.15 times loner than that of the PF method and 3.75 times longer than that of the MS method.

In current markets, the performance-based regulation market in Pennsylvania-JerseyMaryland power pool, i.e. PJM Interconnection Company, is the most profitable one for BESSs. While the BESSs are always assumed to deliver very high performance, an optimal schedule optimizer is proposed including an innovative realistic BESS performance model. The performance model, which characterizes the trade-off between the performance and the power bids in both energy and regulation markets, contributes to the regulation energy scheduling and guarantees BESS performance. The schedule optimizer also models the pricing uncertainties in both markets by generating scenarios according to their respective statistical characteristics. After adopting the proposed optimal scheduling strategy, the cost-performance index (CPI) of the BESSs governed by HiEMS is boosted to $31.70 \%$, compared to around $10 \%$ from only energy market participation. 


\section{Table of Contents}

Dedication . . . . . . . . . . . . . . . . I I

Acknowledegment . . . . . . . . . . . . . . . . . I

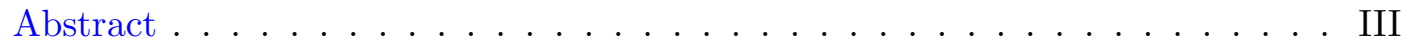

Table of Contents . . . . . . . . . . . . . . . . . . V

List of Figures $\ldots \ldots \ldots \ldots \ldots \ldots \ldots$ IX $\ldots \ldots \ldots \ldots \ldots$

List of Tables . . . . . . . . . . . . . . . . . . XI

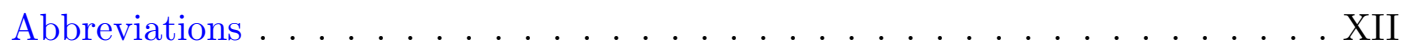

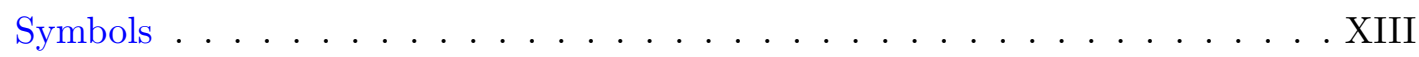

1 Introduction $\quad 1$

1.1 Research Background . . . . . . . . . . . . . . . . . . . . 1

1.2 Motivations . . . . . . . . . . . . . . . . . . 2

1.3 Major Contributions of this Research . . . . . . . . . . . . . . . . . 5

1.4 Organization of this Thesis $\ldots \ldots \ldots \ldots \ldots$

2 Literature Review $\quad 8$

2.1 LFC Techniques . . . . . . . . . . . . . . . . . . . . 9

2.2 Frequency Regulation Market . . . . . . . . . . . . . . . . . . . 12

2.2.1 Market Mechanisms . . . . . . . . . . . . . . . . . . . . . 12

2.2.2 Regulation Performance Criteria . . . . . . . . . . . . . . . 14

2.3 New Resources for Frequency Regulation . . . . . . . . . . . . . . . . . . . 17

2.3.1 BESSs in Frequency Regulation . . . . . . . . . . . . . . . . . 18

2.3.2 Other Demand Side Controllable Devices . . . . . . . . . . . . 19

2.4 Energy Management Systems for Multi-use BESSs . . . . . . . . . . . 20

2.5 Virtual Power Plant Research . . . . . . . . . . . . . . . . . . . . 21

2.6 MPC Strategy . . . . . . . . . . . . . . . . . . . . . 22 
2.6.1 General MPC Knowledge [94] . . . . . . . . . . . . . . 22

2.6.2 MPC in Power System Applications . . . . . . . . . . . . . 25

2.7 Summary . . . . . . . . . . . . . . . . . . 26

3 Studies of Aggregated BESSs for Frequency Regulation $\quad 27$

3.1 Introduction . . . . . . . . . . . . . . . . . . . . 27

3.2 System Configuration _ . . . . . . . . . . . . . . . . 28

$3.2 .1 \quad$ Power System Model . . . . . . . . . . . . . . . . . . . 28

$3.2 .2 \quad$ LFC Model . . . . . . . . . . . . . . . . . . . . . . . . . . . . 30

3.2 .3 BESS Model . . . . . . . . . . . . . . . . . . . . 30

3.3 Rule-based BESS Controller . . . . . . . . . . . . . . . . . . . . . 32

$3.3 .1 \quad$ BESS Aggregation . . . . . . . . . . . . . . . . . 32

$3.3 .2 \quad$ BESS Operation Rules . . . . . . . . . . . . . . . . . . . . 33

3.4 Sizing Analysis with NERC Criterion . . . . . . . . . . . . . . . . 35

3.4.1 Sizing Analysis Using CPS1 and CPS2 . . . . . . . . . . . . . 35

3.4.2 System Frequency Studies with Suggested BESS Penetration . . 38

3.5 BESS Management and Performance Studies . . . . . . . . . . . . . . 41

3.5.1 BESS Effectiveness Analysis . . . . . . . . . . . . . . . . . . 41

3.5.2 Frequency Regulation Participation . . . . . . . . . . . . . 43

3.6 Summary . . . . . . . . . . . . . . . . . . . . . . . . . 47

4 Hierarchical MPC-based EMS for a VPP with BESSs 49

4.1 Introduction . . . . . . . . . . . . . . . . . . . . . . . . 49

4.2 Hierarchical Control Mechanism _. . . . . . . . . . . . . . . . 50

4.2 .1 Primary Control: Lasso MPC . . . . . . . . . . . . . . . . 52

4.2.2 Secondary Control: Economic Oriented MPC . . . . . . . . . . . 53

4.3 Test System Structure and Modeling . . . . . . . . . . . . . . . . . . 54

4.3.1 Lumped Solar System Model . . . . . . . . . . . . . . . . . . . . 54

4.3 .2 Lumped BESS Model . . . . . . . . . . . . . . . . . . . 55

4.3.3 CHP Power Plant Model . . . . . . . . . . . . . . . . . . . 55

4.4 Simulation Results . . . . . . . . . . . . . . . . . . . 56

4.4.1 Simulation Environment . . . . . . . . . . . . . . . 56

$4.4 .2 \quad$ Economic Dispatch . . . . . . . . . . . . . . . . . . . . 57

4.4 .3 System Frequency Control . . . . . . . . . . . . . . . . . . . 58

4.4.4 Dynamic Frequency Regulation . . . . . . . . . . . . . . . . . 58 
4.5 Summary . . . . . . . . . . . . . . . . . . . . 62

5 Hierarchical EMS for BESSs in Multiple Markets $\quad 63$

5.1 Introduction . . . . . . . . . . . . . . . . . . . . 63

5.2 Proposed HiEMS Configuration . . . . . . . . . . . . . . . . . 65

$5.2 .1 \quad$ Schedule Level . . . . . . . . . . . . . . . . . . . 67

5.2.1.1 Prediction Module . . . . . . . . . . . . 67

5.2.1.2 Regulation Assessment Module . . . . . . . . . . . 68

5.2.1.3 BESS Cost Estimation Module . . . . . . . . . . 68

5.2 .2 Dispatch and Dynamic Regulation Levels . . . . . . . . . . . . 69

$5.2 .2 .1 \quad$ Dispatch Level . . . . . . . . . . . . . . . . . . . 69

5.2 .2 .2 Dynamic Regulation . . . . . . . . . . . . . . . 69

5.3 Optimizer Formulations . . . . . . . . . . . . . . . . 70

5.3 .1 First Level Schedule Optimizer . . . . . . . . . . . . . . 70

5.3.1.1 State-space Modeling . . . . . . . . . . . . . . 70

5.3.1.2 Optimization Formulation . . . . . . . . . . . . . 72

5.3 .2 Second level Dispatch Optimizer . . . . . . . . . . . . . . 73

5.3.2.1 State-space Modeling . . . . . . . . . . . . 73

5.3.2.2 Optimization Formulation . . . . . . . . . . . 73

5.4 Simulation Environment . . . . . . . . . . . . . . . . . . . . . . 74

5.5 Parametric Studies . . . . . . . . . . . . . . . . . . . 76

5.5.1 MPC Prediction Horizon Analysis . . . . . . . . . . . . 76

5.5 .2 Pareto Optimality Analysis . . . . . . . . . . . . . 78

5.6 Simulation Studies . . . . . . . . . . . . . . . . . . . . 79

5.6 .1 24-hour Operation Results . . . . . . . . . . . . 79

5.6 .2 CPI Improvement of BESS . . . . . . . . . . . . . 80

5.7 Comparisons . . . . . . . . . . . . . . . . . . . . . . 82

5.7 .1 Optimal Scheduler Comparison . . . . . . . . . . . . . 82

5.7.2 Real-time Performance Comparisons . . . . . . . . . . . . . . . 83

5.7.2.1 Performance Comparisons . . . . . . . . . . . . 83

5.7 .2 .2 BESS Usage and Lifetime . . . . . . . . . . . . 85

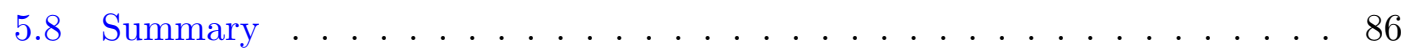

6 BESS Scheduling in Performance-based Regulation $\quad 87$

6.1 Introduction . . . . . . . . . . . . . . . . . . . . . . 87 
6.2 Regulation Simulations . . . . . . . . . . . . . . . . . . 88

$6.2 .1 \quad$ BESS Modeling . . . . . . . . . . . . . . . . . 88

6.2.2 Performance Score Simulations _ . . . . . . . . . . . . . . . . 89

6.3 Optimal Scheduling Formulation . . . . . . . . . . . . . . . . . 91

6.4 Simulation Studies . . . . . . . . . . . . . . . . . . . . . . . . . 94

6.4 .1 Scenario Generation . . . . . . . . . . . . . . . . . 94

6.4 .2 Base Case and Comparisons . . . . . . . . . . . . . . . . 96

6.4 .3 Income Analysis . . . . . . . . . . . . . . . . . . . . 98

6.4 .4 Impacts of Scenario Numbers . . . . . . . . . . . . . . . . . . 99

6.4.5 Impact of PV Penetration . . . . . . . . . . . . . . . . . . . 99

6.5 Summary . . . . . . . . . . . . . . . . . . . 100

7 Conclusions and Future Works 102

7.1 Conclusions . . . . . . . . . . . . . . . . . . . . . . . 102

7.2 Recommendations for Future Works . . . . . . . . . . . . . . . . . . 104

$\begin{array}{ll}\text { References } & 107\end{array}$

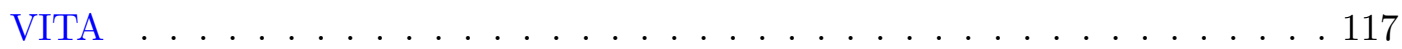




\section{List of Figures}

2.1 Power system model diagram. . . . . . . . . . . . . . . . . . . 9

2.2 Time frames for frequency regulation $[19] \ldots \ldots \ldots$. . . . . . . 10

2.3 AGC control logic in one control area [19] . . . . . . . . . . . . . . 11

2.4 Traditional and dynamic regulation signal generation. . . . . . . . . . . 13

2.5 Normalized traditional and dynamic regulation signals. . . . . . . . . . . . 14

2.6 MPC in current and future control hierarchies [94] . . . . . . . . . . . 22

3.1 Power system frequency model. . . . . . . . . . . . . . . . . . . . . 29

3.2 Thermal power plant simulation model. . . . . . . . . . . . . . . . . . 29

3.3 Block-diagram of AGC system. . . . . . . . . . . . . . . . . . . . 30

3.4 Aggregated multiple BESS system. . . . . . . . . . . . . . . . . 33

3.5 LFC algorithm for aggregated BESS. . . . . . . . . . . . . . . . . 34

3.6 Flowchart of BESS penetration rate analysis. . . . . . . . . . . . 36

3.7 CPS scores with different BESS penetration rates. . . . . . . . . . . . . 37

3.8 Results of system frequency studies with a 15\% BESS penetration. . . . . 39

3.9 Frequency distribution with different BESS penetration rate. . . . . . . . 40

3.10 CPS1 with different PV penetration rate. . . . . . . . . . . . . . . . 41

3.11 Comparison of battery system and slow generator regulation. . . . . . . . 42

3.12 Effectiveness study results with different disturbance power. . . . . . . . . 44

3.13 Singapore frequency data in November of 2012 . . . . . . . . . . . . . . 45

3.14 SOC of storage battery system. . . . . . . . . . . . . . . . 46

3.15 Partial enlarged view of power output of storage battery system. . . . . . 46

4.1 Overall power system structure with VPP. . . . . . . . . . . . . 51

4.2 Structure of a hierarchical MPC controller. . . . . . . . . . . . . . 51

4.3 Test system configuration. . . . . . . . . . . . . . . . . . . 54 
4.4 Non-reheat thermal power plant model. . . . . . . . . . . . . . . . 56

4.5 Hourly PV output and load curve. . . . . . . . . . . . . . . . 56

4.6 Dispatch strategy at the first hour of the day. . . . . . . . . . . . . . . 57

4.7 Simulation results from 9:00 am to 10:00 am. . . . . . . . . . . . . 59

4.8 Simulation results from 15:00 to $16: 00 \ldots \ldots$. . . . . . . . . . . . 60

4.9 Set point deviations for the Generator and BESS from 9:00 to 9:30. . . . 61

4.10 Frequency deviation curve with and without VPP . . . . . . . . . . 61

5.1 Proposed HiEMS integration and its working time scheme. . . . . . . . . 66

5.2 Major function modules and data exchanges in the proposed HiEMS. . . . 67

5.3 A Singaporean 14-bus radial distribution network. . . . . . . . . . . . . 74

5.4 Simulation pricing data. . . . . . . . . . . . . . . . . . . . 75

5.5 Schematic diagram of system simulations. . . . . . . . . . . . . 76

5.6 MAPE with regards to different prediction lead time values. . . . . . . . . 77

5.7 Parametric studies for Pareto optimality. . . . . . . . . . . . . . 78

5.8 System consumption schedule, and real-time curves with and without HiEMS. . . . . . . . . . . . . . . . . . . . . 80

5.9 Simulation results for the 24 -hour period. . . . . . . . . . . . . . 81

5.10 CPI scores with varying regulation regulation participation rates and PV penetration rates. . . . . . . . . . . . . . . . 82

5.11 Controller output and respective BESS SOC curves from 3:30 to 7:00. . . 84

5.12 Individual BESS equivalent usage cycles for three controllers. . . . . . . . 85

6.1 A general BESS model for response testing. . . . . . . . . . . . . . . . . 88

6.2 Work flow for performance score simulations. . . . . . . . . . . . . . . 90

6.3 Performance scores for different regulation capacity settings. . . . . . . . . 90

6.4 Regulation price distributions of a 638-day database. . . . . . . . . . . . . 94

6.5 Probabilistic forecast results for USEP . . . . . . . . . . . . . . . . 95

6.6 Dynamic performance and piecewise linear fitting results. . . . . . . . . 95

6.7 24-hour schedules with dynamic performance and constant score. . . . . . 97

6.8 24-hour simulation SOC results. . . . . . . . . . . . . . . . . . . 98

6.9 CPI and score for different PV penetration settings. . . . . . . . . . . 100 


\section{List of Tables}

1.1 Unit prices for different batteries . . . . . . . . . . . . . . . 4

2.1 A summary of US regulation markets . . . . . . . . . . . . . . . . 12

3.1 Parameters for the sizing analysis tests . . . . . . . . . . . . . . 36

3.2 CPS1 scores for varying $\mathrm{M}$ values . . . . . . . . . . . . . . . . . 38

3.3 Parameters for system frequency simulation . . . . . . . . . . . . 38

3.4 Comparison of frequency regulation effective level . . . . . . . . . . . . . . 43

3.5 Comparison of usage life cycle and total non-response time $\ldots . . . . .47$

4.1 Standard deviation values without and with VPP . . . . . . . . . 62

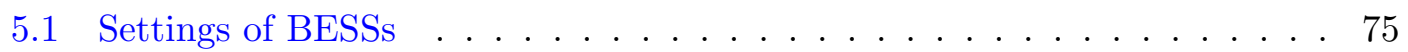

5.2 Estimated annual revenue with varying lead time values . . . . . . . . . 77

5.3 Optimizer parameters . . . . . . . . . . . . . . . . . . . 79

5.4 Comparisons with a fully centralized scheduler . . . . . . . . . . 83

5.5 Comparisons between three control methods . . . . . . . . . . . . . . 85

6.1 Comparisons between dynamic and constant score . . . . . . . . . . . 98

6.2 Comparisons between different scenario numbers for a 238-day period . . 99 


\section{Abbreviations}

ACE Area Control Error

AGC Automatic Generation Control

ANN Artificial Neural Network

BESS Battery Energy Storage System

CAISO California Independent System Operator

CHP Combine Heat and Power

CPI Cost Performance Index

CPS Control Performance Standard

DAC Dynamic Annualized Cost

DER Distributed Energy Resources

DR Demand Response

DRS Down Regulaion Servie

ERCOT Electric Reliability Council of Texas

HiEMS Hierarchical Energy Management System

LFC Load Frequency Control

MPC Model Predictive Control

NERC North American Electricity Reliability Corporation

NYISO New York Independent System Operator

PID Proportional Integral Derivative

PJM Pennsylvania-New Jersey-Maryland Interconnection

PSCC Power System Control Centre

PV PhotoVoltaic

RES Renewable Energy Sources

RTU Remote Terminal Unit

SOC State Of Charge

URS Up Regulaion Servie

USEP Uniform Singapore Energy Price

VPP Virtual Power Plant 


\section{Symbols}

A

$A_{S}$

B

$B_{f}$

$B_{S}$

$B_{\text {sys }}$

C

$C_{S}$

$C_{\text {bat }, i}$

$c_{b a t, i j}$

$c_{k W}, c_{k W h}$

$c_{e, t}^{s}$

$c p_{t}^{s}$

D

$E_{i}^{\min }, E_{i}^{\max }$

$E_{i j}^{\min }, E_{i j}^{\max }$

$E_{i, t}$

$E_{i j, t}$

$F_{H P}$

$H_{p}, H_{p}^{\prime}$
System matrix of the system state space model.

Weighting parameter for precision score $\left(S_{P}\right)$ when calculating performance score $S$.

Input matrix of the system state space model.

Frequency bias of the control area $(\mathrm{MW} / \mathrm{Hz})$.

Weighting parameter for correlation score $\left(S_{C}\right)$ when calculating performance score $S$.

Sum of frequency bias of all the control areas within the system $(\mathrm{MW} / \mathrm{Hz})$.

Output matrix of the system state space model.

Weighting parameter for delay score $\left(S_{D}\right)$ when calculating performance score $S$.

Battery cost for the $i^{\text {th }}$ group BESS $(\$ / \mathrm{MWh})$.

Battery cost for the $j^{t h}$ BESS in the $i^{t h}$ group ( $\$ / \mathrm{MWh}$ ).

BESS power investment $(\$ / \mathrm{kW})$ and energy investment $(\$ / \mathrm{kWh})$.

Energy market price at time $t$ in scenario $s(\$ / M W h)$.

Regulation capacity clearing price $\left(R E G_{C C P}\right)$ at time $t$ in scenario $s$ $(\$ / M W)$.

Damping coefficient of the system loads $(\mathrm{MW} / \mathrm{Hz})$.

Minimum and maximum energy stored in the $i^{\text {th }}$ BESS group (MWh).

Minimum and maximum energy stored in the $j^{\text {th }}$ BESS in the $i^{\text {th }}$ group (MWh).

Energy stored at time $t$ for the $i^{\text {th }}$ BESS group (MWh).

Energy stored at time $t$ for the $j^{\text {th }}$ BESS in the $i^{\text {th }}$ group (MWh).

The fraction of the total power generated by the high pressure section in the power plant.

Prediction horizon of schedule optimizer and that of dispatch optimizer. 
$h_{e}, h_{r}$

$h_{m, t}$

$h_{t}$

$i, i j$

$K_{I}$

$K_{P}$

$M$

$m$

$P_{i}^{\min }, P_{i}^{\max }$

$P_{i, t}^{e, d}, P_{i, t}^{e, c}$

$P_{i, t}^{r}$

$P_{i j}^{\min }, P_{i j}^{\max }$

$P_{i j, t}^{d}, P_{i j, t}^{c}$

$P_{t}^{\text {load }}, \bar{P}_{t}^{\text {load }}$

$P_{t}^{\text {grid }}, \bar{P}_{t}^{\text {grid }}$

$P_{t}^{r e g}$

$P_{t}^{P V}, \bar{P}_{t}^{P V}$
Time interval in markets (hour) and time duration for regulation signals (seconds).

Regulation energy scheduled at time $t$ when it falls to segment $m$ (MWh/MW).

Regulation energy scheduled at time $t$ (MWh/MW).

BESS group index and individual BESS index in the $i^{\text {th }}$ group.

Integral coefficient for PI controllers.

Proportional coefficient for PI controllers.

System inertia constant (second).

Segment index for piecewise linearization.

Minimum and maximum power set point for the $i^{\text {th }}$ BESS group (MW).

Dispatched group discharge and charge for the $i^{\text {th }}$ BESS group at time $t$ (MW).

Power bid for the $i^{t h}$ group at time $t$ in regulation market (MW).

Minimum and maximum power set point for the $j^{\text {th }}$ BESS in the $i^{\text {th }}$ group (MW).

Real-time discharge and charge for the $j^{\text {th }}$ BESS in the $i^{\text {th }}$ group at time $t(\mathrm{MW})$.

Real-time load consumption at time $t$ (MW) and average hourly load consumption at time $t$ (MW).

Real-time power consumption from the energy market (MW) and scheduled hourly power consumption from the energy market (MW).

Real-time regulation signal from the regulation market at time $t$ (MW).

$\mathrm{PV}$ real-time power output at time $t(\mathrm{MW})$ and average hourly PV power output at time $t(\mathrm{MW})$.

$P_{t i e} \quad$ Real-time telemetered tie-line power flow (MW).

$P_{\text {tie,sched }} \quad$ Scheduled tie-line power flow (MW).

$p^{s} \quad$ Probability for scenario $s$. 
$p p_{t}^{s}$

$R_{G}$

$S_{C}$

$S_{D}$

$S_{P}$

$T_{G}$

$T_{C H}$

$T_{R H}$

$t, t^{\prime}$

$t_{\sigma}$

$U_{m, t}$

$u(t)$

$\bar{u}_{h}$

$y(t)$

$\epsilon_{1}, \epsilon_{2}$

$\theta_{\text {reg }}$

$\lambda_{\text {reg }}$

$\sigma_{t}$

$\Delta f$

$\Delta P_{L}$

$\triangle P_{P V}$

$\Delta P_{G E N}$
Regulation performance clearing price $\left(R E G_{P C P}\right)$ at time $t$ in scenario $s(\$ / \mathrm{MW})$.

The droop parameter in primary frequency regulation.

Correlation score.

Delay score.

Precision score.

Scenario index.

Time constant of the governor in the generator.

The time constant of the main inlet volumes and steam chest in a thermal power plant.

The time constant of the reheater in a thermal power plant.

Time step index for the schedule optimizer and dispatch optimizer.

The time when the maximum correlation $\left(\sigma_{t}\right)$ happens.

Binary variable to represent whether the BESSs operate in the $m$ th segment.

Regulation signal sent to the regulation unit at time $t$.

Hourly average value of the regulation signal.

Actual unit response at time $t$ when calculating unit performance.

1-minute and 10-minute average deviation of the benchmark signal for regulation performance tests.

Regulation performance constraint $(\leq 1)$.

Regulation participation rate $(\leq 1)$.

Correlation between the 5-minute regulation signal and the unit response with $t$ seconds shifting.

Frequency $(f)$ deviation from the nomination value $\left(f_{0}\right)(\mathrm{Hz})$.

Load consumption change (MW).

Power change of the PV generation (MW).

Power change of the thermal power plant (MW). 
$\triangle P_{B E S S} \quad$ Power change of the BESS (MW).

$\Delta P_{\text {tie }}$

The tie-line power error out of the control area (MW). 


\section{Chapter 1}

\section{Introduction}

In this chapter, the background knowledge of this thesis is introduced. The motivations and contributions are summarized. And the organization of this thesis is described at the end of this chapter.

\section{$1.1 \quad$ Research Background}

The generation capacity of renewable energy sources (RESs) is growing in today's power systems, such as photovoltaic (PV) and wind energy. RESs can help to handle the ever-expanding electricity demand, and are beneficial in decreasing the dependency on the fossil fuel and reducing the carbon emission. As a tropical nation with sufficient solar irradiation, Singapore has an average annual solar irradiation of $1,580 \mathrm{kWh} / \mathrm{m}^{2} /$ year, which is around $50 \%$ more than that of temperate countries. It is stated that the PV generation is reaching $350 \mathrm{MWp}$ by 2020 , which is $5 \%$ of the estimated peak demand [1].

As the penetration rate increases, RESs will cause power imbalance as a result of their intermittency. Besides, RESs are mostly not frequency-sensitive components, i.e. non-responsive of system frequency deviation. Therefore, when the percentage of RESs generation expands, the system equivalent inertia decreases. Consequently, rapid and drastic frequency fluctuations are more likely to take place, which is challenging for traditional power plants to regulate due to their slow dynamics. Therefore, the RESs penetrated power system has stricter requirements for both the energy capacity and the 
response speed of reserve and regulation resources.

Recently, battery energy storage systems (BESSs) are more frequently utilized in power system applications. Since no rotation mass is involved, their advantage over the traditional resources is that BESSs are capable of responding to fast fluctuating signals, and that they are very flexible in adopting different control mechanisms. In most cases, the only limit is often the ramp rate of the power converter devices which connect the BESSs to the grid. However, considering the high cost of BESSs, the optimal sizing and controller design issues are crucial to improving the performance and the costeffectiveness of a grid-scale BESS or aggregated BESSs.

Additionally, with the deregulation of the electricity market, there have been some markets being able to take frequency regulation bids from resources beyond a certain size, instead of solely dependent on traditional power plants. Some newly emerging alternative regulation resources include BESSs and controllable loads of various kinds, which are usually small in capacity and dispersedly located in distribution networks. Therefore, those resources can be aggregated as a virtual power plant (VPP) to enter the market. Extended research works are going on regarding the participation of BESSs in the electricity markets, aiming to gain the maximum revenue.

\subsection{Motivations}

Most BESS sizing strategies aim at maximizing the financial profits of the stakeholders. However, from the system operator's point of view, the sizing issue of the BESS aggregation in the system should also be studied based on the overall system control performance to ensure a high quality regulation service [2]. The overall frequency performance with different penetration rates of BESS is to be analyzed. And the effectiveness of the BESSs in frequency regulation is addressed based on the long-term system performance. Frequency performance is evaluated by Control Performance Standard 1 (CPS1) and Control Performance Standard 2 (CPS2) proposed by North American Electricity Reliability Corporation (NERC) [3].

Regarding the control mechanism of BESSs, there have been many research works addressing the control of BESSs which participate in regulation or stabilizing the os- 
cillations of the system frequency $[4,5,6]$. In most studies, the load frequency control (LFC) scheme for BESSs is based on the classical droop controllers or PID controllers in response to the system frequency deviation. The coordination between BESSs and other slow-dynamic regulation resources are conducted by filtering the regulation signal. Slow-varying components of the signal are sent to units with limited ramp capability, such as thermal power plants, and fast-varying components are sent to BESSs.

However, these commonly studied control schemes have a few drawbacks when applied on BESSs. Firstly, droop controllers and PID controllers are unable to coordinate with multiple regulation resources according to their different responding characteristics. In the long-term system simulations in [7], BESSs with fast response ability can work against the power plant with slow dynamics, especially during rapid and drastic changes. Therefore, a more sophisticated controller is required to optimize the coordination between the resources. Secondly, these regulation controllers are not able to take system frequency dynamics and BESS state of charge (SOC) dynamics into consideration. By incorporating these system dynamic models into the controller, these system states can be controlled to follow the desired operation trajectories or stay within the permitted ranges. In particular, the energy storage capacity of BESS is limited, and thus the amount of stored energy in BESSs should be controlled to avoid saturation and depletion, which leads to non-response time during operations. Thirdly, when using droop/PID controllers as well as filters for regulation signal generation, the power ratings of different regulation resources are implemented as power limit modules (limiter circuits) to enforce the regulation signal range [8]. This mechanism does not help to fully exploit the regulation capability among all types of resources, and may not meet the total system regulation requirement during certain operation situations. For this reason, power rating limits should be considered when the commands are generated, instead of after the droop/PID controllers and filters.

With regard to the three technical challenges listed above, model predictive control (MPC) is an excellent candidate for BESS control. MPC is a widely used control strategy for real-time optimal control problems with multiple variables and constraints. By customizing the optimization objective and system constraints, the MPC controller is able to coordinate between different resources and be aware of system states, such as frequency and SOC of BESSs, in order to achieve an optimal operation. 
Table 1.1: Unit prices for different batteries

\begin{tabular}{l|c|c||c}
\hline \multirow{2}{*}{} & \multicolumn{2}{|c||}{ Cost } & \multirow{2}{*}{ Power rating } \\
\cline { 2 - 3 } & $\$ / \mathrm{kW}$ & $\$ / \mathrm{kWh}$ & \\
\hline Vanadium & $600(\sim 1500)$ & $100(\sim 1000)$ & $10 \mathrm{~kW}-3 \mathrm{MW}$ \\
\hline Lead-acid & $300(\sim 600)$ & $200(\sim 400)$ & $0-20 \mathrm{MW}$ \\
\hline Lithium-ion & $1200(\sim 4000)$ & $600(\sim 2500)$ & $0-100 \mathrm{~kW}$ \\
\hline *US dollar as the monetary unit
\end{tabular}

Although BESSs are gaining increasing research interests in power system applications for their fast-response ability and control flexibility, it is still difficult for BESSs to be profitable due to high battery costs [9], even with the proposed optimal sizing scheme [10]. General pricing information from the literature $[11,12,13]$ is summarized in Table 1.1. In order to promote the economic value of BESSs, BESSs are maneuvered to participate in market operations. For BESS's multiple markets participation, there are mainly two issues described below.

Firstly, an optimal coordination between multiple market operations is required. Extensive research works on energy arbitrage of BESSs have been conducted. A number of papers studied the optimization formulations based on multiple available markets $[14,15]$. Recently, a more financially rewarding performance-based regulation service is considered by [16]. However, in [16], the realistic BESS performance is not considered. Consequently, the BESS capacity is not fully utilized and will fail to get the optimal reward.

Secondly, due to the minimum capacity requirement from the market regulator, several BESSs need to be aggregated to reach the minimum threshold to enter the market. When multiple BESSs are connected in the same network, coordination between the units helps to fully exploit the aggregation storage capacity, and optimize the real-time operation. In the current literature $[17,18]$, the coordination of multiple BESSs often does not consider respective characteristics and SOC of the BESSs, hence resulting in unbalanced usage of the BESSs. 


\subsection{Major Contributions of this Research}

After introducing the research background and the emerging issues, the major contributions are concluded as follows:

1. The sizing issue of an aggregated BESS is studied based on long-term system control performance [2]. The recommended penetration rate of BESSs in the power system is analyzed using an iterative approach according to the performance. The recommended BESS penetration rate in the power system is analyzed using an iterative approach according to the NERC performance indices. Taking the investment cost into consideration, a $5 \%$ to $15 \%$ BESS penetration rate is recommended for the test system. The analysis method can also be applied to other systems. By incorporating the recommended BESS in the power grid, the system frequency deviation distribution is improved based on the simulation results.

2. A hierarchical MPC-based controller is proposed for a centralized VPP coordination. The proposed controller is composed of two levels, with the primary controller for regulation and the secondary linear economic oriented controller for economic dispatch, aiming to coordinate the power balance among PV power output, BESS power commands, and load consumption. The controller is capable of stabilizing system frequency within the permitted range during heavy load and peak solar generation periods.

3. A hierarchical EMS (HiEMS) is further developed for a distribution network with distributed BESSs participating in multiple markets as one entity, in order to achieve better cost-effectiveness. With the proposed HiEMS, the BESS aggregation can obtain optimal schedules; ensure the real-time performance; and thus gaining the maximum profit from the participated markets. And as a result of BESSs coordination considering BESS lifetime, the time of first replacement for BESSs is prolonged.

4. To further enhance the benefit of BESSs, an optimal scheduling strategy is devised focusing on the most profitable performance-based regulation market as well 
as the energy market. After adopting the proposed optimal scheduling strategy, the cost-performance index (CPI) of the BESSs governed by HiEMS is boosted to $31.70 \%$, compared to around $10 \%$ from only energy market participation.

\subsection{Organization of this Thesis}

This thesis is organized as follows:

Chapter 1 provides the basic background information for this research, and describes the issues relating to the performance of BESSs in frequency regulation, control schemes for BESSs, and market participations of BESSs. This chapter also summarizes the motivation and contributions of this research.

Chapter 2 does a literature review on related research topics, including LFC mechanism, regulation market mechanism and key market rules, as well as new regulation resources. The energy management of BESSs is also discussed in this chapter. Last but not the least, recent VPP research works and MPC algorithm are presented.

Chapter 3 simulates and analyses BESSs participation in frequency regulation. The aggregation controller rules for the BESSs are introduced. The regulatory effectiveness of the BESSs is discussed based on the simulation results. The sizing analysis of the proposed BESS is done to improve the system performance.

Chapter 4 models the VPP components used in the latter case studies, including the power system frequency dynamics model, battery model, PV energy system model, and the LFC controller model. The proposed hierarchical MPC controller is introduced in details, including the Lasso MPC algorithm used in the primary controller. Optimal problem settings are discussed in details. System simulation and result analysis are also included.

Chapter 5 introduces the HiEMS work flow and major functionalities. The detailed problem formulation in the optimizers is presented. Parametric studies are conducted to obtain optimal controller settings. Simulation studies for optimal scheduling and realtime operation are carried out. In addition, Chapter 5 also includes a complete analysis and comparison of results. 
Chapter 6 introduces the optimal scheduling strategy for BESSs participating in energy and performance-based regulation markets. Scenario-based optimization formulation is presented. Scenario generation method is also illustrated. Several comparisons and analytical studies are included towards the end of this chapter.

Chapter 7 concludes the whole thesis and discusses future work recommendations. 


\section{Chapter 2}

\section{Literature Review}

With the growing penetration rate of the RESs, the power system requires more regulation capacity and better control schemes for the connected distributed energy resources (DERs). In this chapter, research methodologies and related studies will be introduced. The literature review covers the following aspects:

- An introduction of the basic theories of LFC and the common methodologies used in previous and ongoing research

- A summary of current frequency regulation market mechanisms to facilitate DER market participation studies

- DERs' control strategies and their impacts on system frequency, especially the ones for BESSs

- Energy management for BESSs participating in multiple market operations

- A brief introduction of the commonly used VPP concept for DER aggregations

- General knowledge of MPC control strategy chosen in this research and its implementations in power system applications 


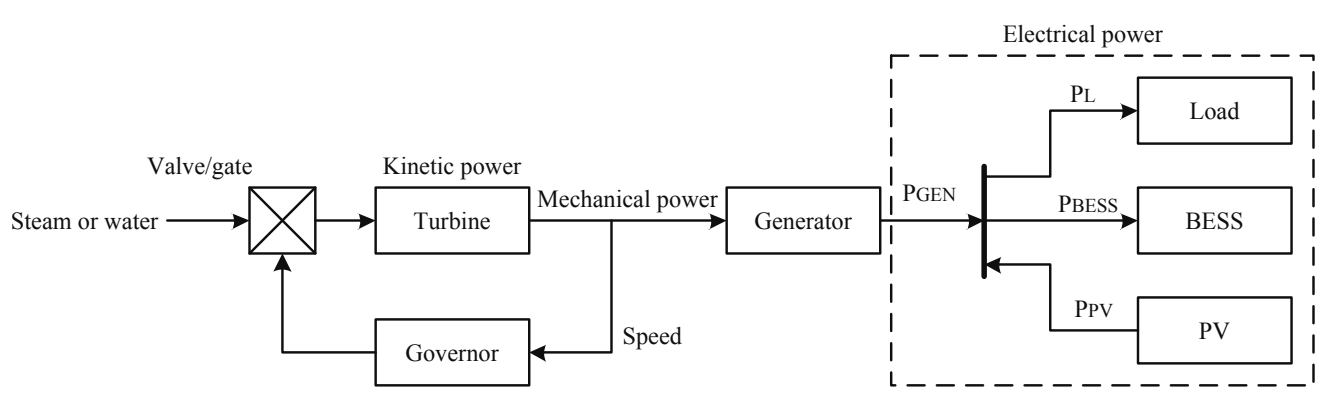

Figure 2.1: Power system model diagram.

\subsection{LFC Techniques}

In system modeling, the large utility grid components are simplified as a slowresponding thermal generation plant, which maintains the grid frequency. As depicted in Fig. 2.1, when there is a mismatch between the mechanical power produced by the prime mover and the electrical power generated by the generator, the rotation mass of the generator will use the stored kinetic energy to compensate for the mismatch. As a result, the rotation speed of the generator will change, and in turn, the system frequency will deviate from the nominal value.

When dealing with small frequency fluctuations around the nominal grid frequency, the complicated power system dynamic is linearized around its normal operation point, and is represented by a low-order system model. The overall generator-load inertia dynamic relationship between the incremental mismatch power and the frequency deviation can be expressed by the system swing equation in (2.1).

$$
\Delta P_{G E N}(t)+\Delta P_{P V}+\Delta P_{B E S S}(t)-\Delta P_{L}(t)=M \frac{d \Delta f(t)}{d t}+D \Delta f(t)
$$

The swing equation can be used in system frequency simulations, and is also widely used in model-based modern controller design.

In power systems, the frequency is regulated by on-line generation units normally with fast ramping capacity. The whole regulation process consists of actions in three timescales shown in Fig. 2.2: primary control, secondary control, and tertiary control.

\section{Primary frequency control}




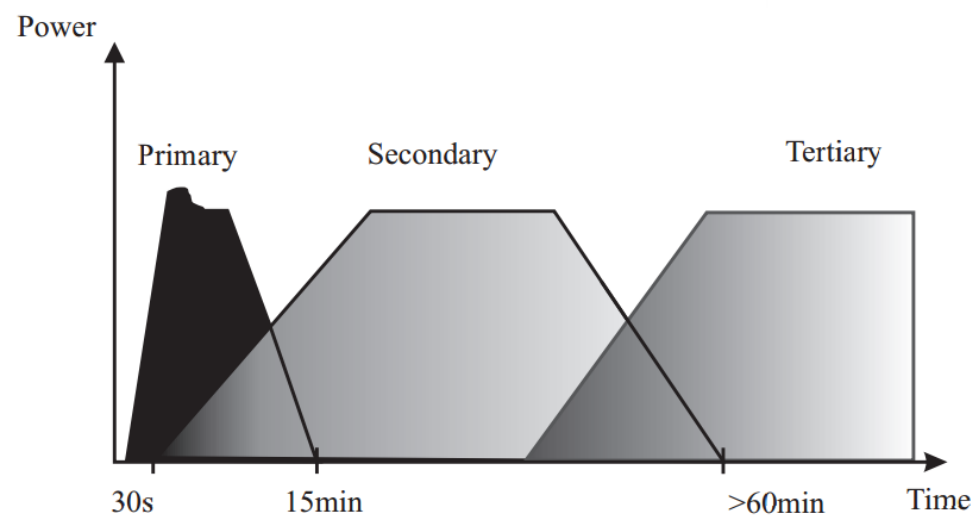

Figure 2.2: Time frames for frequency regulation [19].

The speed governor reacts to frequency deviations, and changes the mechanical power input of the prime mover. This speed governing process, together with the load damping effect and other frequency-responsive entities with droop characteristic are all included in primary frequency control. However, primary frequency control is unable to restore the system frequency to the nominal value.

\section{Secondary frequency control}

Secondary frequency control takes action by adjusting the set point of the generator. This is the main task of automatic generation control (AGC), which is located in large synchronous generators. Besides, AGC also refers to the regulatory control of the local power system control center (PSCC), which sends regulation signal every 2 to 4 seconds. The control scheme is plotted in Fig. 2.3. The area control error (ACE) signal for the control area is generated as in (2.2).

$$
\begin{aligned}
A C E & =\left(P_{\text {tie }}-P_{\text {tie,sched }}\right)+B_{f}\left(f-f_{0}\right) \\
& =\Delta P_{t i e}+B_{f} \Delta f
\end{aligned}
$$

\section{Tertiary frequency control}

When the system operator observes that the primary and secondary controls have been activated constantly for a prolonged period, the system operator will manually activate tertiary frequency control to free the primary and secondary control reserve. 


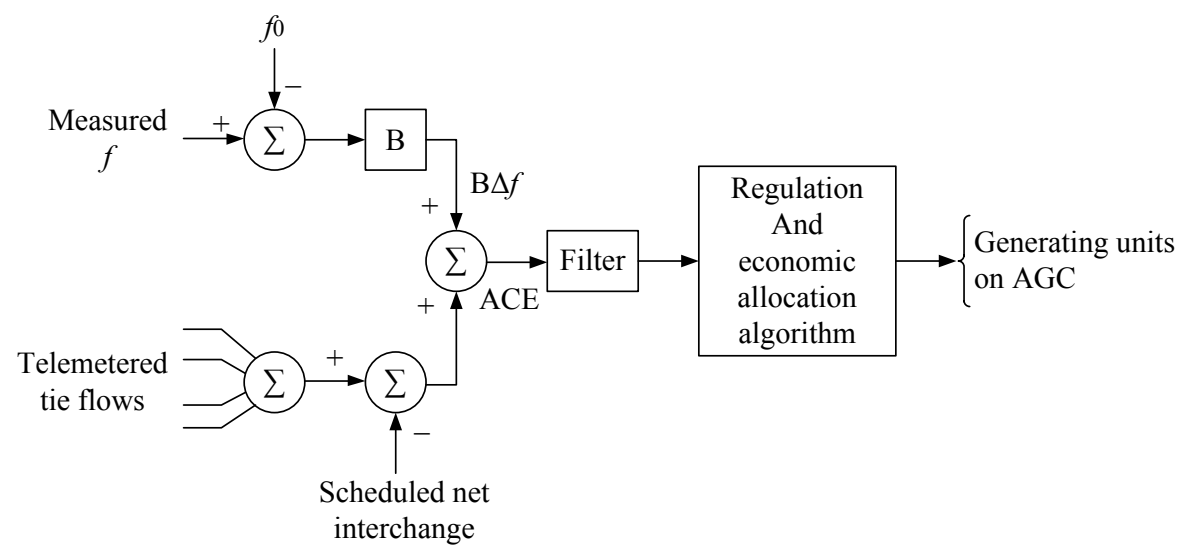

Figure 2.3: AGC control logic in one control area [19].

on average slightly more than 8 Modern control theories are implemented in LFC scheme to achieve a better system performance.

Starting from the 1970s, optimal control theory is implemented in secondary LFC scheme $[20,21]$, where the control variables are optimized to achieve the optimal frequency trajectory to minimize the cost function. Reference [18] formulates the multi-area LFC problem using differential game theory, where the optimal control for each area is generated considering the collaboration or competition among the interconnected areas.

Later from the 1990s, robust control theory for LFC has been studied to deal with the uncertainties in the system parameter and disturbances [22, 23]. Especially after the growing numbers of DERs, robust controllers are necessary to compensate for the uncertainties [24]. Meanwhile, adaptive control method is adopted by LFC schemes to cope with the parametric uncertainties $[25,26,27,28]$. Furthermore, artificial intelligent methods, such as neural network and fuzzy logic, are also applied to LFC schemes $[29,30,31]$. In [32], a fuzzy logic controller is developed to provide adaptive participation factors for the network, with a particle swarm optimization-based on-line tuning mechanism. A nonlinear control method sliding mode control is used in [33] to increase the robustness against the uncertainties in the system. In [34], an MPC controller is developed for the Nordic network with hydro power being the major LFC resource. The proposed MPC controller improves the system performance, and also allows for a more flexible LFC scheme considering network constraints and enabling coordination between resources. A similar MPC controller is proposed for plug-in hybrid electric vehicles in response to the system frequency signal [35]. 
Table 2.1: A summary of US regulation markets

\begin{tabular}{|c|c|c|c|}
\hline Market & $\begin{array}{c}\text { Regulation bid } \\
\text { (MW) }\end{array}$ & $\begin{array}{c}\text { Revenue } \\
\text { accounting }\end{array}$ & $\begin{array}{c}\text { Minimum } \\
\text { resource size }\end{array}$ \\
\hline \hline $\begin{array}{c}\text { PJM } \\
\text { traditional } \\
\text { \& dynamic) }\end{array}$ & $\begin{array}{c}\text { double-directional } \\
\text { regulation }\end{array}$ & $\begin{array}{c}\text { capacity reward } \\
\text { and } \\
\text { performance reward }\end{array}$ & $0.1 \mathrm{MW}$ \\
\hline ERCOT & URS and DRS & capacity reward & $0.1 \mathrm{MW}$ \\
\hline CAISO & URS and DRS & $\begin{array}{c}\text { Capacity reward } \\
\text { proportional to mileage }\end{array}$ & $0.5 \mathrm{MW}$ \\
\hline ISO-NE & $\begin{array}{c}\text { double-directional } \\
\text { regulation }\end{array}$ & $\begin{array}{c}\text { Capacity reward } \\
\text { proportional to score }\end{array}$ & $1 \mathrm{MW}$ \\
\hline NYISO & $\begin{array}{c}\text { double-directional } \\
\text { regulation }\end{array}$ & $\begin{array}{c}\text { Capacity reward } \\
\text { proportional to mileage }\end{array}$ & $1 \mathrm{MW}$ \\
\hline
\end{tabular}

\subsection{Frequency Regulation Market}

\subsubsection{Market Mechanisms}

The National Energy Market of Singapore comprises a wholesale market and a retail market. To introduce competition in both markets, energy producers or consumers (prosumers) above a certain size threshold can sell or purchase electricity subject to the real-time pricing scheme. In the real-time market, the market clearing engine generates energy, reserve, and regulation prices once every 30 minutes. Regulation providers in the Singapore market are paid monthly based on the uniform regulation price, without performance standards involved.

Although currently there is no energy bidding from the demand side in the Singapore electricity market, many electricity markets do accept regulation bids from qualified DERs, such as BESSs and demand response units. Some of them require up or down regulation service bids (URS or DRS), and some require double-directional regulation within the given capacity. In North America, such markets include Electric Reliability Council of Texas (ERCOT) [36], California Independent System Operator (CAISO) [37], and New York Independent System Operator (NYISO) [38]. The market mechanisms are reviewed in Table $2.1[39,40]$.

However, with the increasing penetration rate of intermittent RESs, the grid requires 


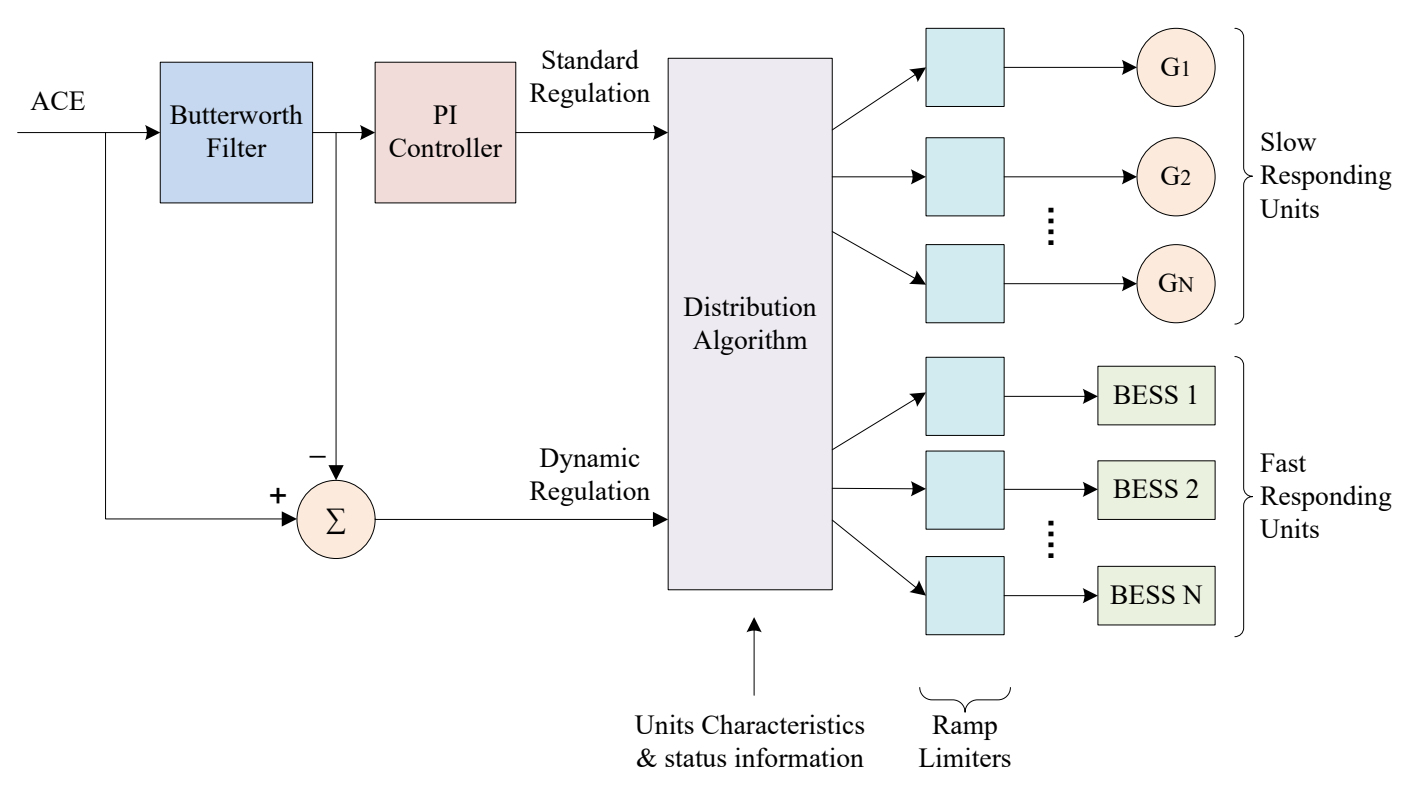

Figure 2.4: Traditional and dynamic regulation signal generation.

faster regulation capacity. PJM has introduced a performance-based dynamic regulation market, and participants can choose to respond to the slower-varying traditional regulation signal or the dynamic regulation signal. In the updated PJM regulation rationale [8], the residue of control signal and traditional regulation signal is provided by dynamic regulation signal. The signal generation scheme is plotted in Fig. 2.4. More controller details can be found in reference [8].

The traditional regulation signal is processed with a set of low-pass filters and PI controllers. Similarly, the dynamic regulation signal is also derived from the ACE through a high-pass filter and a PI controller to obtain the dynamic regulation signal. Historical traditional and dynamic regulation signals for one hour are plotted in Fig. 2.5.

The dynamic regulation signal is distributed to very fast responding units. These units will be rewarded with high payments, which are normally around three times higher than that for providing traditional regulation $[18,41]$. The payment is also subject to real-time performance. Therefore, participating in the performance-based regulation is very profitable for BESS units, compared to participation in the energy market only $[16,42]$. 


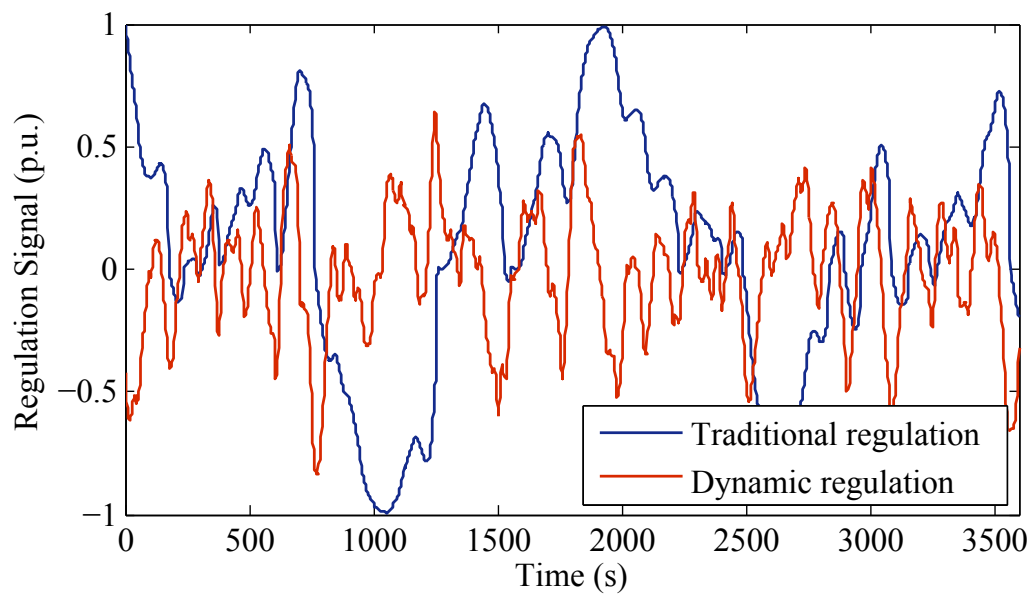

Figure 2.5: Normalized traditional and dynamic regulation signals.

\subsubsection{Regulation Performance Criteria}

In system operation, frequency disturbances within a certain range are tolerable, and frequency regulation units are not forced to respond strictly according to the commands. However, certain level of compliance is required. In this section, two categories of performance criteria are presented from the system operator's point of view and participating unit's point of view respectively.

\section{CPS1 and CPS2 criteria}

$C P S 1$ measures the effectiveness of the frequency regulation within a control area. For each minute, a compliance factor $C F 1$ is calculated as in (2.3). $C F 1$ represents the ratio of the covariance between the actual frequency deviation and the ACE deviation, to the variance of a benchmark signal. The one-minute average variance of the benchmark signal is referred to as $\epsilon_{1}^{2}$, whose value is set and reviewed by NERC.

$$
C F 1=\frac{1}{\epsilon_{1}^{2}}\left(\frac{{\overline{A C E_{1}}}_{1}}{B_{f}}\right) \times \Delta f_{1}
$$

The $C P S 1$ evaluation considers the average $C F 1$ for 12 consecutive months, and is represented as a percentage value. The CPS1 formulation is shown in (2.4). During system operations, the CPS1 value is reported every month using a rolling 12-month average. In [43], $C P S 1$ is used as the criterion to optimize the area AGC gain when the RES penetration increases greatly in the future. 


$$
C P S 1=100 \times\left(2-\overline{C F 1}_{12 \text { Month }}\right)
$$

The minimum requirement for $C P S 1$ is $100 \%$, and it has no upper limit. However, for a single control area without tie-line power exchange, the maximum score possible is $200 \%$, because the product of $A C E$ and $\Delta f$ will always be a positive number.

Nevertheless, $C P S 1$ alone is not sufficient in evaluating the system frequency regulation performance. A control area could still score high in CPS1 by relying on its neighboring control areas, without being efficient in following its own power mismatches. This will result in excessive tie-line power exchanges. Therefore, the other criteria CPS2 is assessed to limit the power flows from the interconnected control areas.

$C P S 2$ evaluates the 10 -minute average $\mathrm{ACE}$, which is denoted as $\overline{A C E}_{10}$. Within a month, at least $90 \%$ of all the $\overline{A C E}_{10}$ values should not exceed the limit $L_{10}$, which is defined in (2.5):

$$
L_{10}=1.65 \epsilon_{10} \sqrt{B_{f} \cdot B_{\text {sys }}}
$$

where $B_{\text {sys }}$ is the sum of frequency bias of all the control areas within the system, and $\epsilon_{10}$ is the 10-minute average deviation of the benchmark signal.

To represent the comparison in a mathematical formulation, the ratio of $\overline{A C E}_{10}$ to $L_{10}$ is defined as the second compliance factor $C F 2$ in (2.6).

$$
C F 2=\frac{\left|\overline{A C E}_{10}\right|}{L_{10}}
$$

The overall calculation procedure is summarized in (2.7) and (2.8).

$$
\begin{gathered}
R=\frac{\text { Number of intervals with } C F 2>1}{\text { Total number of intervals }} \\
C P S 2=100(1-R)
\end{gathered}
$$

The specific values of the factors involved are related to the actual system size. The detailed computation process could be found in [44]. 


\section{Unit Performance Evaluation Criterion}

Electricity market companies have their specific rules to calculate the performance of each unit. In this research, the performance is evaluated using the PJM criteria [45].

The performance score consists of three performance scores considering three different aspects in the unit response:

- Precision score $\left(S_{P}\right)$

- Correlation score $\left(S_{C}\right)$

- Delay score $\left(S_{D}\right)$

$S_{P}$ indicates how accurate the unit can react to the commands, while the systematic error of the unit is captured by $S_{C}$ and $S_{D}$. $S_{C}$ represents the resemblance of the shapes between the responses and the commands, and $S_{D}$ shows the general time lag when the maximum resemblance happens. Detailed definitions are given as follows.

$S_{P}$ is calculated in (2.9), measuring the average errors between the unit command and its response.

$$
S_{P}=1-\frac{1}{n} \sum_{t=1}^{n}\left|\frac{y(t)-u(t)}{\bar{u}_{h}}\right|
$$

where $y(t)$ is the actual unit response; $u(t)$ is the regulation signal; and $\bar{u}_{h}$ is the hourly average value of the regulation signal.

$S_{C}$ in (2.10) measures the maximum correlation between the 5-minute regulation signal and the unit response with $t$ seconds shifting $\sigma_{t}$ as defined in (2.11).

$$
\begin{gathered}
S_{C}=\max \left(\sigma_{0}, \sigma_{1}, \ldots, \sigma_{t}\right) \quad \forall t \in[0,10 s, 20 s, \ldots, 300 s] \\
\sigma_{t}=\frac{\sum_{i=t}^{t+300 \mathrm{~s}}\left(u_{i}-\bar{u}_{h}\right)\left(y_{i}-\bar{y}_{h}\right)}{\sqrt{\sum_{i=t}^{t+300 \mathrm{~s}}\left(u_{i}-\bar{u}_{h}\right)^{2} \sum_{i=t}^{t+300 \mathrm{~s}}\left(y_{i}-\bar{y}_{h}\right)^{2}}}
\end{gathered}
$$

After gaining the maximum correlation $S_{C}, S_{D}$ is calculated from the time when the maximum correlation happens $\left(t_{\sigma}\right)$ in $(2.12)$. 


$$
S_{D}=\left|\frac{t_{\sigma}-300 s}{300 s}\right|
$$

The final composite score $S$ in $(2.13)$ is a weighted average of $S_{P}, S_{C}$ and $S_{D}$ :

$$
S=A_{S} \cdot S_{P}+B_{S} \cdot S_{C}+C_{S} \cdot S_{D}
$$

where parameters $A_{S}, B_{S}$, and $C_{S}$ are decided by the market governor. In most cases, their values are equal, i.e. $A_{S}=B_{S}=C_{S}=1 / 3$.

\subsection{New Resources for Frequency Regulation}

As the RES penetration rate increases and RESs are usually highly intermittent and uncertain, the system equivalent inertia is decreasing and rapid frequency fluctuations are prone to take place. Therefore, integrating RESs into the utility grid could raise severe stability issues, especially in system frequency.

Currently, several grid codes impose requirements for renewable power plants [46, 47, 48]. In order to provide frequency regulation, the renewable power plants are required to decrease their output power proportional to the frequency increase, and disconnect from the grid during over or under frequency contingencies outside the permitted range. This one-directional regulation is not sufficient for LFC commands. Variable-speed wind turbines are capable of providing primary frequency control by using the stored kinetic energy to emulate virtual inertia [49] or pitch control to regulate their real power output $[50,51]$. However, the wind turbine deliberately works below the maximum power output in order to provide double-directional regulation, which will lead to a waste of energy.

Energy storage systems are often coupled with RESs. They are utilized as buffers to enable smoother RES output profiles. Various kinds of energy storage systems are discussed in literature. Commonly used storage systems are flywheels [52], BESSs [53, 54], and hybrid energy storage systems [55, 56]. Recently, electrical vehicles connected to grid are also maneuvered to serve as storage systems $[57,58]$. Detailed control strategies for BESSs and other regulation resources are discussed in the following sections. 


\subsubsection{BESSs in Frequency Regulation}

In this section, the control schemes for BESSs in frequency response have been summarized. This research focuses on BESSs, because they are fast-acting devices which can arrest rise and drop in frequency. In $[4,5,6]$, BESSs participate in regulation, stabilizing the oscillations and improving system frequency performance. Furthermore, with the cost reduction of batteries in recent years, grid-scale BESSs are becoming more promising to participate in the system regulation services.

In early research studies, a signal which is proportional frequency error or ACE is directly used as the BESS control signal to study the ability of BESSs to follow the regulation commands $[7,59,60,61]$. BESSs are proven to be more effective than the conventional power plants in improving system frequency. However, as a storage device, the battery holds limited amount of energy. As a result, BESSs may have non-response time when the stored energy is depleted or saturated. Moreover, when dispatching regulation resources using traditional participation factors, the fast-responding ability of BESSs is not fully utilized. Therefore, more sophisticated control schemes are developed according to the characteristics of the BESSs. There are mainly two categories of control schemes described below.

\section{SOC-based participation factor modification}

The power output of the BESS is regulated considering the SOC information. As long as the system frequency dynamics are within the limit, the BESS commands are modified to absorb more/less energy to maintain the SOC level. In [59], other than the base case where frequency error is used as the control input, the authors also developed a more advanced operation strategy by scheduling the BESS to charge or discharge during "noncritical frequency windows", in order to maintain its SOC with a safe margin. In $[62,63]$, the SOC information is combined with the ACE signal to form the input for the BESS, so that the power command corrects the system frequency as well as the BESS SOC. A SOC modified participation factor method for energy storage systems in islanded microgrid was proposed in [64]. In [65], an MPC controller is utilized to manipulate the BESS power output considering the future system frequency response and future SOC. However, in the real-time operation, the proposed Grey predictor for frequency can be very hard to get accurate predictions. Therefore, further robustness studies are advised. 
In $[66,67]$, the participation factor of the BESS is gradually decreasing with the saturation/depletion of the BESS. Coupled RESs are also used to compensate for the long term energy deviation as in [68].

\section{Filtering and decomposition}

As the fast fluctuating component of the regulation signal is a "zero-energy" service [69] and the BESS is capable of dealing with fast fluctuating signals, various kinds of decomposition techniques are used to eliminate the long-term energy deviation signal from the frequency error. The BESSs are only responsible for short-term fluctuations. As such, the system uses BESSs in the most effective way. Meanwhile, the BESSs seldom suffer from energy constraints when only responding to short-term fluctuations. In [70, 71], filters with different time constants are used to generate suitable control signals for BESSs. In [72], a model based decomposition method is used to separate the signal within the suitable bandwidth.

\subsubsection{Other Demand Side Controllable Devices}

Commonly studied controllable devices are often equipped with energy storage ability, such as electrical vehicle (EV) batteries (electrical energy), water heaters [73], refrigerators [74] or air-conditioners [75] (thermal energy storage), or equipment with variable speed drives whose power consumption is a function of the rotation speed, such as variable speed heat pumps [76] and fan motors in the building ventilation system [77, 78].

Additionally, electrical vehicles connected to the grid (V2G) are also regarded as an alternative regulation resource. The control scheme is highly similar to the control strategies for BESSs, and further constrained by the users' driving and charging behaviors [79]. In the work of [71], the LFC signal is sent to a number of EVs and heat pump water heaters, where the water heaters take the slow dynamic components and EVs take the fast dynamic ones.

There are a series of studies examining the system frequency transient under the influence of controllable loads or demand response (DR) devices. In [80], the short-term frequency dynamic responses in less than 20 seconds are simulated for both stable and 
unstable systems. Controllable loads are modeled as an increased load-damping coefficient to represent the frequency-sensitiveness, and it is proved to exacerbate system frequency fluctuations which lead to unstable systems. However, the paper assumes that the load control program is carried out locally and the central frequency regulator is unaware of the load control actions. With regard to this issue, a separate DR control loop is introduced in [81]. The DR and the traditional power plant are controlled by the LFC through a power-sharing factor. The frequency dynamic is improved with the DR response. Another research work improves the system frequency dynamic in an isolated network with a time-varying load-damping coefficient controller, controlling the electrolytic aluminum load in the local factory [82].

\subsection{Energy Management Systems for Multi-use BESSs}

In order to promote the economic value of BESSs, BESSs are managed to participate in market operations. There have been many research works addressing energy arbitrage using BESSs, therefore this work will focus on BESSs conducting multiple services. For energy arbitrage, reference [83] provides a thorough optimal bidding framework for BESS in energy market participation.

For multi-use BESSs, an optimal coordination between multiple market operations is required. A number of papers studied the optimization formulations based on multiple available markets. Reference [14] optimizes the BESS bidding in the day-ahead energy and spinning reserve market, and reference [15] further includes the ramp-up and rampdown frequency regulation service in an attempt to increase the revenue. Moreover, a more financially rewarding performance-based regulation service is considered by [16].

However, unlike energy arbitrage, the real energy consumption by ancillary services (regulation, spinning reserve, and non-spinning reserve) is uncertain and cannot be accurately predicted. This will lead to difficulties in optimal scheduling. For that reason, more realistic energy storage models are required for optimal scheduling, specifying the real energy consumption when providing certain services. In [84], a "high-fidelity dispatch model" is proposed by introducing a "deployment factor", to represent the average energy consumption of ancillary services. In [16], the amount of energy consumed by 
regulation is also set as a constant with a large margin (15 minutes or $0.25 \mathrm{MWh} / \mathrm{MW}$ ) to ensure its high performance (above 0.9 out of 1 ), while the actual energy requirement remains uncertain.

In real market revenue accounting, the final regulation revenue is usually proportional to the performance as well as the power bids. Therefore, when scheduling the BESS energy capacity, keeping a large energy margin will limit the amount of power that BESS can bid into the markets; in other words, there is a trade-off between the performance and the power bids. A constant setting of the energy consumption cannot fully utilize the BESS capacity and will fail to get the optimal reward.

\subsection{Virtual Power Plant Research}

In traditional power systems, DERs and BESSs are often connected to the grid passively in a "fit and forget" measure. The power system operator is unable to control or dispatch these DERs, which means that they are not taking part in market operations, even if the total installed capacity is large enough to participate. In the meantime, when the grid consumption grows, the system has to increase its generation capacity, resulting in extra capital investment and construction time. This results in capacity redundancy and degraded system efficiency. Besides, due to the minimum capacity requirement from the market regulator, several resources need to be aggregated to reach the minimum threshold to enter the market.

VPP serves as a powerful technique to aggregate multiple generation units or microgrids within an information network to operate holistically as one entity [85]. In [86], several buildings with controllable heating systems are aggregated to provide demand response service in the market. A PV power plant in [87] is aggregated with controllable loads (ice machines) to provide a dispatchable output. Demands, wind turbines, and energy storage devices are coordinately controlled to act as a VPP in day-ahead and the real-time markets $[88,89]$. A similar VPP with DERs, BESSs, and electricity consumers is studied in [90], and the bidding strategy also includes the market clearing process to optimize the VPP bids. A decision tree method is used in [91] to dispatch the aggregated controllable loads in the VPP. Heuristic optimization is also used to deal 
Current practice

\begin{tabular}{|c|}
\hline $\begin{array}{c}\text { Plant-wide static set-point optimization } \\
\text { (daily) }\end{array}$ \\
\hline $\begin{array}{c}\text { Set-point optimization at unit level } \\
\text { (hourly) }\end{array}$ \\
\hline $\begin{array}{c}\text { Predictive control } \\
\text { (Logic, Overrides, Decoupling, } \\
\text { Exception handling) }\end{array}$ \\
\hline \hline $\begin{array}{c}\text { Local loop controllers } \\
\text { (P, PI, PID) }\end{array}$ \\
\hline Actuators \\
(Valve servos etc.)
\end{tabular}

Future trend

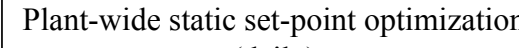
(daily)

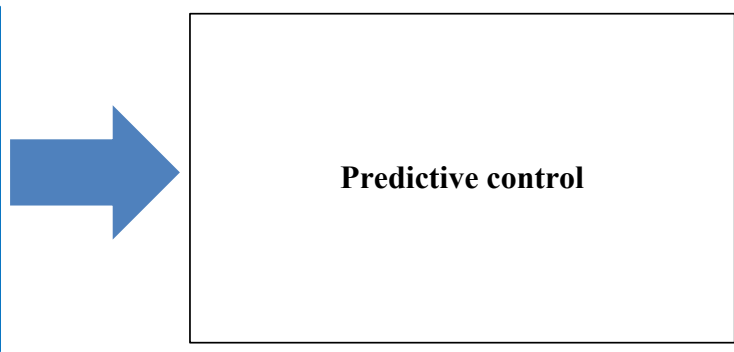

Actuators

(Valve servos etc.)

Figure 2.6: MPC in current and future control hierarchies [94].

with the nonlinear VPP dispatch problem in [92]. Genetic algorithm which optimizes the nonlinear dispatching issue is used in [93] .

\subsection{MPC Strategy}

Fig. 2.6 demonstrates the current practice for control hierarchy and the future trend. In the traditional process control hierarchy, predictive control is usually used between the long-term steady-state optimization and the simple local controller, in the place of logic or rule-based controllers. This control layer is crucial, when some border conditions happen, and thus the local loop controller is not able to respond effectively. Therefore, the predictive control layer is supposed to deal with those exceptional conditions in a systematic and optimal way. However, the future trend in MPC research aims to be more versatile, including control objectives from local loop to short-term steady-state optimization.

\subsubsection{General MPC Knowledge [94]}

In MPC, a state-space model of the system will be used to predict the future states and outputs, starting from the current time $k$ till the end of the prediction horizon $k+H_{p}$. A linearized discrete-time model can be represented in the form of (2.14): 


$$
\left\{\begin{array}{l}
\mathbf{x}(k+1 \mid k)=A \mathbf{x}(k)+B \mathbf{u}(k) \\
\mathbf{y}(k)=C \mathbf{x}(k)+D \mathbf{u}(k)+W \boldsymbol{\omega}(k)
\end{array}\right.
$$

where $\mathbf{x}$ is the state vector; $\mathbf{u}(k)$ is the input vector; $\boldsymbol{\omega}$ is the disturbance vector; and $\mathbf{y}$ is the output vector. System matrices $A, B, C, D$, and $W$ will be defined later depending on specific system modeling.

Future states trajectories $\hat{\mathbb{X}}$ and future control inputs $\hat{\mathbb{U}}$ are defined as follows:

$$
\begin{aligned}
& \hat{\mathbb{X}}=\left[\hat{\mathbf{x}}(k+1 \mid k)^{T}, \ldots, \hat{\mathbf{x}}\left(k+H_{p} \mid k\right)^{T}\right]^{T} \\
& \hat{\mathbb{U}}=\left[\hat{\mathbf{u}}(k+1 \mid k)^{T}, \ldots, \hat{\mathbf{u}}\left(k+H_{u} \mid k\right)^{T}\right]^{T}
\end{aligned}
$$

With the defined state-space model, $\hat{\mathbb{X}}$ can be predicted by the current measured states $\mathbf{x}(k)$ and $\hat{\mathbb{U}}$, by substituting the future states using the state-space model (2.14) iteratively as in (2.17).

$$
\begin{aligned}
\hat{\mathbf{x}}(k+1 \mid k) & =A \mathbf{x}(k)+B \hat{\mathbf{u}}(k \mid k) \\
& \vdots \\
\hat{\mathbf{x}}\left(k+H_{p} \mid k\right) & =A^{H_{p}} \mathbf{x}(k)+A^{H_{p}-1} B \hat{\mathbf{u}}(k \mid k)+\ldots+A^{H_{p}-H_{u}} B \hat{\mathbf{u}}\left(k+H_{p}-1 \mid k\right)
\end{aligned}
$$

At the beginning of every control time step $k$, an optimization program in the form of (2.18) will be executed to generate the future optimal control inputs $\hat{\mathbb{U}}$ over the control horizon $H_{u}$. The formulation of the optimization objective $V$ can be customized to reflect desirable objectives for the future trajectories of the system states and control inputs, while respecting constraints on allowable trajectories. The objective function is described below.

$$
\min _{\mathbf{u}} V(\hat{\mathbb{X}}, \hat{\mathbb{U}})
$$


subject to

$$
\begin{aligned}
& \mathbf{x}(k+t \mid k)=A \mathbf{x}(k+t-1 \mid k)+B \mathbf{u}(k+t \mid k) \\
& \mathbf{u}(k+t \mid k) \in \mathcal{U}, \forall t \in\left[0, H_{u}\right] \\
& \mathbf{x}(k+t \mid k) \in \mathcal{X}, \forall t \in\left[0, H_{p}\right] \\
& \mathbf{x}\left(k+H_{p} \mid k\right)=\mathcal{X}_{f}
\end{aligned}
$$

where $\mathcal{U}$ and $\mathcal{X}$ are the feasible region of $\mathbf{u}$ and $\mathbf{x}$ respectively, and $\mathcal{X}_{f}$ is the final state constraints of the optimization.

Although a series of control inputs $\hat{\mathbb{U}}$ are calculated, only the first input $\hat{\mathbf{u}}(k \mid k)$ is applied to the system. In the next time step, the optimization will be performed again, on the basis of new measurements, thus creating a feedback mechanism.

MPC controllers differ from one another in the prediction methods and the objective function formulations. The prediction models can be system state-space models based on mathematical modeling or time series models based on historical data analysis. For the objective functions, MPC is able to achieve various control targets by deliberately including different variables in the objective function. There are two common types of elements in objective function formation:

\section{Quadratic penalties}

Quadratic state deviations are often used in tracking problems, and help to optimize the state trajectory to follow its reference signals as close as possible. Quadratic decision variables help to minimize the control efforts needed to accomplish the required control action and provide smoother outputs.

\section{Linear penalties}

The one-norm of the decision variables is in fact giving the control input an extra cost to start, preventing a certain unit from being actuated too frequently [95]. 


\subsubsection{MPC in Power System Applications}

Several research works have been done in economic dispatch to minimize operational costs [96] and to maximize battery lifetime [97]. In [98], the authors work on an MPCbased EMS for a DG integrated distribution system, controlling both the reactive power and the active power. Generation rate constraint is also included in the MPC scheme [99]. A decentralized primary frequency MPC controller is devised to control a battery energy storage system, without considering load frequency signal from the distribution system operator [100]. Recently, a hierarchical MPC is designed to synthesize different levels of control or multiple control areas [101]. Kennel et al. constructed a smart grid EMS (both economic dispatch and load frequency control), coordinating power plants, consumers, PV panels and electrical vehicles [79], without considering the governor and turbine dynamics in the power plants.

In this thesis, the proposed HiEMS uses MPC due to its ability to adapt in the following three aspects:

\section{Zone control of the SOC}

One of the major advantages of using MPC is its ability to deal with multi-variable optimizations with constraints. When the system SOC model is included in the MPC prediction model, the MPC controller is able to control the SOC within the permitted range, even though the BESS model is not providing very accurate SOC values. This zone control can be easily achieved using the MPC formulation, but quite difficult with other control methodologies [102].

\section{Adaptive power set point adjustment}

MPC in this application is able to adapt to the varying system dynamics. In current control hierarchy, the local set point is adjusted by low-level local controllers. Local controllers are typically proportional-integral (PI) controllers, or occasionally PID controllers with derivative action. However, in real system operations, an adaptive dispatch scheme is necessary to guarantee a better performance, when the SOC levels of the BESS aggregations are constantly changing. In such situations, predictive control could be implemented to produce adaptive set points according to the most updated system 
measurement and system dynamics. The predictive controller can contain different time scales operations itself, ranging from longer term set point optimization to shorter term real time adjustment [94].

In this research, the EMS performs set point adjustment on different timescales, from half-an-hourly system level optimization to minute-wise dynamic dispatch for a lower level control.

\section{Prediction error tolerance}

In the MPC formulation, each control input from solving the optimization repetitively is nontrivial especially with the presence of prediction errors. For example, intuitively speaking, the scheduler should be making more profit when the prediction horizon is longer, since more future information is known ahead of time and better decisions can be made. However, when perfect prediction is not attainable, prediction errors can result in deviations from the optimal schedule, which in return, can harm the total profit. Therefore, to reach a comparatively optimal strategy, parametric studies should be carried out when involving different prediction technologies in the MPC formulation.

\subsection{Summary}

This chapter prepared the theories and methodologies for power system frequency analysis and simulation, as well as controller design. As the focus of this research, BESSs will be modeled as a part of the power system. The impact of BESSs on system frequency will be simulated using traditional and new control schemes, and then assessed under general performance criteria in Chapters 3 and 4 . When endeavoring to gain more economic value, BESSs can be aggregated into VPPs to participate in profitable markets. Related EMS studies will be elaborated in Chapter 5 and Chapter 6. 


\section{Chapter 3}

\section{Studies of Aggregated BESSs for Frequency Regulation}

\subsection{Introduction}

When studying the grid-connected BESSs, most BESS sizing strategies aim at maximizing the financial profits of the stakeholders. In [59, 103], the size of the BESS is optimized to maximize the total income of the studied microgrid system, considering the initial capital cost of the battery. In this chapter, the sizing issue of an aggregated BESS is studied based on system control performance [2]. The overall frequency performance with different penetration rates of BESS is analyzed. Frequency performance is evaluated by $C P S 1$ and $C P S 2$ proposed by NERC [3]. Frequency domain simulations are conducted to demonstrate the system responses with the suggested BESS capacity and PV penetration. A proper procedure for BESS sizing is presented to ensure a long-term high quality regulation service, considering the rated power and capacity constraints of BESSs.

Another contribution in this chapter is the BESS performance studies. Firstly, the response effectiveness of the BESS is analyzed given different levels of power disturbances. The BESS is more effective than the traditional generator in general, with the effective index ranging from 1 to 10. Secondly, the performance of the BESS aggregation rules are tested using real system frequency dynamics captured by a smart meter installed on-site. Both software simulations and hardware implementations are carried out. 
Furthermore, for utility grid applications, large-scale storage is often not affordable for a single organization. Existing small capacity BESSs could be aggregated to a largescale entity [104]. The aggregated entity is often referred to as a VPP. VPP components are dispatched and coordinated via an information and communication network. Several VPP projects are implemented in Europe, clustering various types of resources [105, 106]. Nevertheless, the above mentioned VPP projects also focus on the economy objectives, rather than the system performance. Therefore, a decentralized BESS controller is designed to fulfill the performance requirement.

The rest of the chapter is organized as follows: Section 3.2 describes the system and battery model used in the simulations. Section 3.3 introduces the aggregation controller rules for the BESSs. In Section 3.4, sizing analysis of the proposed BESS is done to improve the system performance. Section 3.5 presents the BESS performance analysis with simulation results from the effectiveness study and the BESS response tests. Section 3.6 summarizes the highlights of the whole chapter.

\subsection{System Configuration}

The block diagram of the studied control area is presented in Fig. 3.1. The large utility grid components are simplified as a slow-dynamic thermal generation plant and a BESS. System voltage is maintained by the existing reactive power compensators, decoupled to a certain extent from the frequency regulation issue caused by active power imbalance.

\subsubsection{Power System Model}

The dynamic relationship of the overall generator-load inertia between the incremental power mismatch and the frequency deviation can be expressed by the system swing equation in (3.1), using per unit as the measurement unit. This chapter deals with frequency fluctuations around the nominal grid frequency. Therefore, the power system dynamic response is linearized around its normal operation point, and represented by a low-order system model as depicted in Fig. 3.1. 


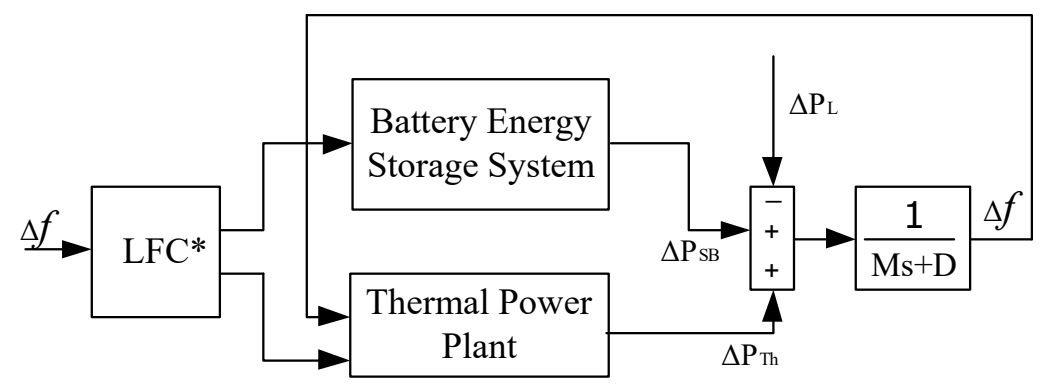

*incorporates the time delay of the communication link

Figure 3.1: Power system frequency model.

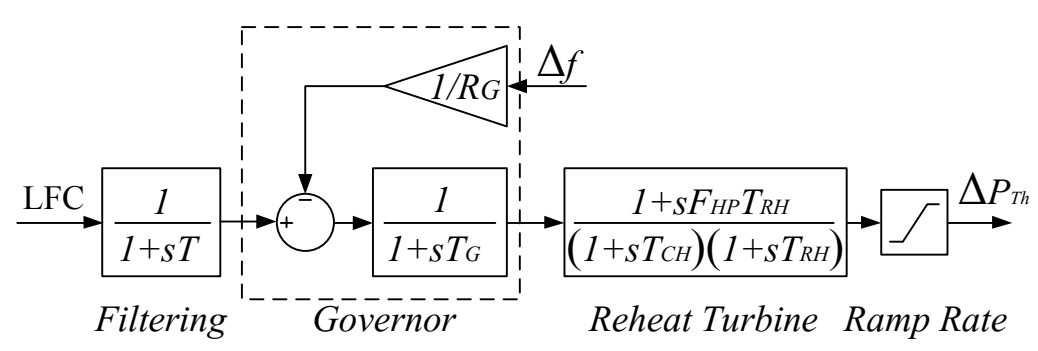

Figure 3.2: Thermal power plant simulation model.

$$
\Delta P_{T h}(t)+\Delta P_{S B}(t)-\Delta P_{L}(t)=M \frac{d \Delta f(t)}{d t}+D \Delta f(t)
$$

where $\Delta f$ is the per unit frequency deviation based on the system nominal frequency; $\Delta P_{T h}$ is the power change of the thermal power plant; $\Delta P_{S B}$ is the power change of the BESS; $\Delta P_{L}$ is the load change; $M$ is the inertia constant and $D$ is the damping coefficient of the system loads.

The thermal power plant participates in primary and secondary frequency control in the simulation. Major blocks are shown in Fig. 3.2 [107]. The reheat turbine model is used to represent the power plant dynamics. $T_{C H}$ is the time constant of the main inlet volumes and steam chest; $T_{R H}$ is the time constant of the reheater; and $F_{H P}$ is the fraction of the total power generated by the high pressure section in the power plant. The primary frequency regulation is represented by a droop block $1 / R_{G}$ and the time constant $T_{G}$ of the governor in the generator. The LFC or secondary frequency control signal is discussed in details in the next section. 


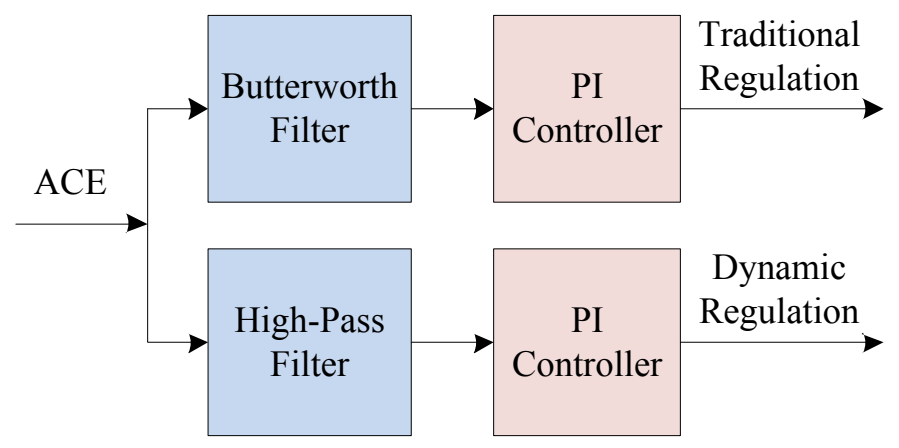

Figure 3.3: Block-diagram of AGC system.

\subsubsection{LFC Model}

The LFC command is calculated based on the ACE. The commonly used AGC algorithm in Energy Management System (EMS) is summarized in Fig. 3.3. The standard regulation signal is derived from ACE, processed with a set of Butterworth and PI controllers, which helps to eliminate the fast-varying component and the noises from measurement and communication procedures. In a traditional power system control area, where there are multiple generation units participating in the regulation, the standard regulation signal is distributed to each unit according to their participation factors, considering their capacity and ramp rate values [19].

The dynamic regulation signal is also derived from the ACE through a high-pass filter and a PI controller. The dynamic regulation signal is distributed to very fast responding units, such as BESSs, which barely have time delay and are capable of immediate response.

The total regulation requirement is distributed to the units based on their participation factors with consideration of their ramp rates, capacity and the current status.

A small constant time delay of the communication link is applied to the LFC in the simulations [7].

\subsubsection{BESS Model}

Lithium ion (Li-ion) batteries are chosen as the storage device in this chapter. When coupled with a PV energy system, PV generation can be dispatched to charge up the 
storage battery when there is sufficient solar irradiation [108]. With energy stored, the Li-ion battery is utilized to respond to AGC's dispatch commands.

\section{Frequency Domain Modeling}

In system analysis, the battery is simplified as a first-order transfer function $G_{B}$ in (3.2). The time delay in communication and control signal processing is modeled as the time constant $T_{B}$ in the transfer function.

$$
G_{B}(s)=\frac{1}{1+T_{B} s}
$$

\section{Time Domain Modeling}

To further simulate the real time response of the battery, the time domain model is developed. The equations for charging and discharging events are shown as in (3.3). $P_{t}^{E, d}$ represents the discharging power of the battery at time $t$, and $t \in[0, T] . P_{t}^{E, c}$ is the power of the battery charged by the grid or the PV energy system. The energy stored in the battery at time $t$ is noted as $C(t)$. $\Delta t$ is the update time step of the stored energy. $\eta_{d}$ and $\eta_{c}$ are the discharge efficiency and charging efficiency respectively. The battery also subject to power limits and energy limits from (3.4) to (3.6).

$$
\begin{aligned}
& \text { Discharge : } C(t+1)=C(t)-\Delta t P_{t}^{E, d} / \eta_{d} \\
& \text { Charge : } C(t+1)=C(t)+\Delta t P_{t}^{E, c} \eta_{c}
\end{aligned}
$$

subject to the following battery constraints:

- Power limits:

$$
\begin{aligned}
& 0 \leq P_{t}^{E, d} \leq P_{E}^{d, \max } \\
& 0 \leq P_{t}^{E, c} \leq P_{E}^{c, \max }
\end{aligned}
$$

- Stored energy limits:

$$
C_{\min } \leq C(t) \leq C_{\max }
$$


- Starting and ending limits:

$$
C(0)=C(T)=C_{S}
$$

where $P_{E}^{d, m a x}$ is the maximum discharge rate; $P_{E}^{c, \max }$ is the maximum charge rate; $C_{\min }$ and $C_{\max }$ are the minimum and maximum energy stored in the battery bank; and $C(0)$ is the initial energy inside the battery bank, which is equal to $C_{S}$. For the energy balance of the BESS, the final energy stored inside the battery bank $C(T)$ is set the same as the initial stored energy.

\subsection{Rule-based BESS Controller}

\subsubsection{BESS Aggregation}

Although battery technology is advancing rapidly in recent years, the capital cost of a large BESS is still quite high while the capacity of BESS has to reach a certain scale to influence system frequency significantly. The VPP concept which aggregates multiple small-scale BESSs into a large-scale entity is applied.

The distributed BESSs are coordinated to drive the ACE to zero. The total control demand is allocated to each BESS based on the individual participation factor of the BESS, which is proportional to its rated power. This distribution process is briefly illustrated in Fig. 3.4. The communication network is responsible for transporting control and measurements. The EMS located at the PSCC sends regulation commands to BESS Aggregation Center, and the BESS Aggregation Center delivers charging or discharging signals to every BESS. Conversely, the BESS Aggregation Center collects measured power data from each BESS and submits the data to the EMS located at the PSCC. Communication between EMS and BESS Aggregation Center is via the use of a remote terminal unit (RTU). 


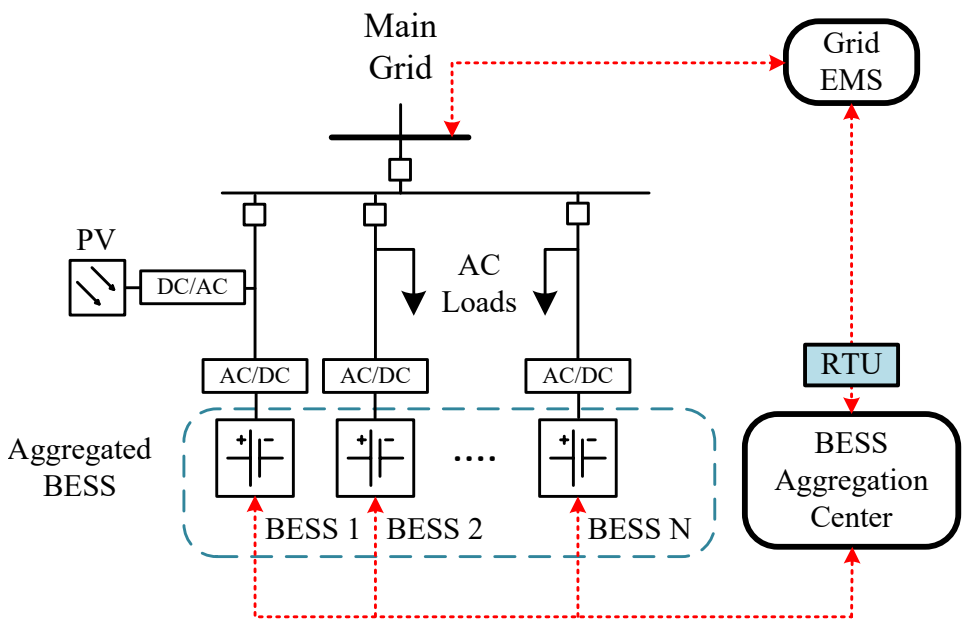

Communication Network …..... Distribution Network

Circuit breakers

$\square$

Figure 3.4: Aggregated multiple BESS system.

\subsubsection{BESS Operation Rules}

The BESS participating in frequency regulation should be able to maintain the frequency around its nominal value, which is $50 \mathrm{~Hz}$ in Singapore. The control signal will be processed by the BESS Aggregation Center, and then passed on to the distributed Battery Management Systems (BMSs). The BMS will control the output of the BESS based on the charge/discharge signal. The whole process of the frequency regulation algorithm is shown in Fig. 3.5. Moreover, the SOC of the individual BESS is also utilized to constrain the set point of BESS.

The participation factor of an aggregated BESS is limited within the maximum power capacity, and is calculated according to Rule 1 below:

Rule 1: If the frequency change is equal to or greater than $0.2 \mathrm{~Hz}$, the BESS will help to reduce the absolute value of ACE by working at the maximum power rating.

Based on Rule 1, the BESS will work at its maximum power rating to arrest the ACE deviation. If the participation factor is set at $\rho$, we have

$$
\begin{aligned}
& A C E * \rho=P^{\max } \\
& \Rightarrow \rho=P^{\text {max }} /\left(\Delta f^{\text {threshold }} * B_{f}+\Delta P_{\text {tie }}\right)
\end{aligned}
$$




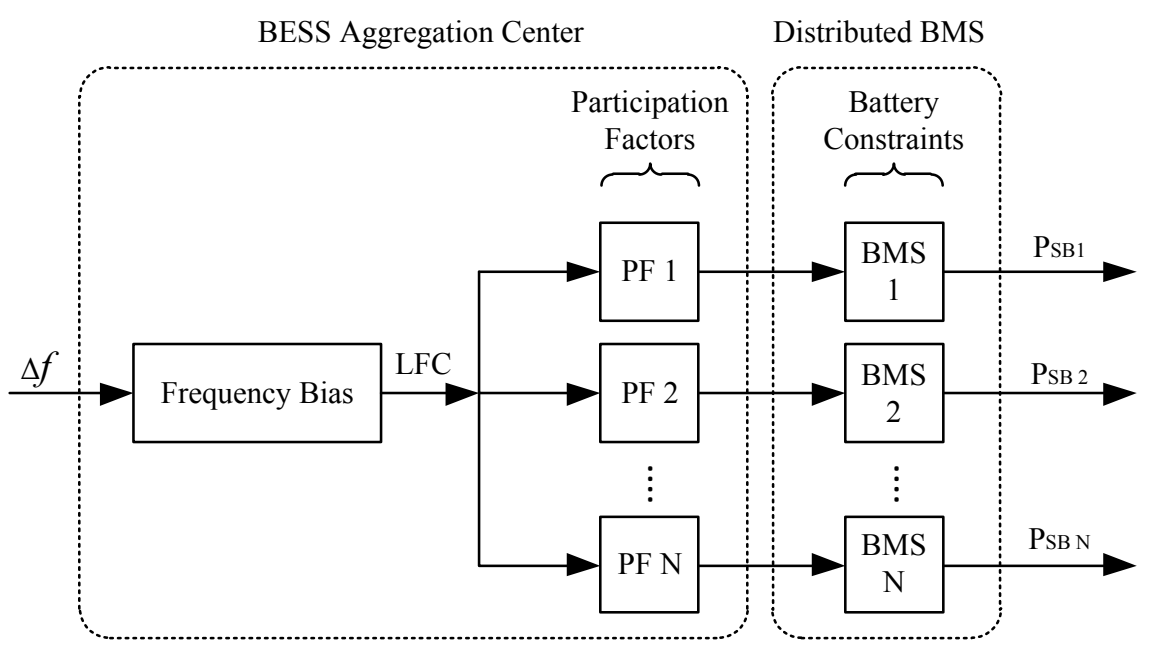

Figure 3.5: LFC algorithm for aggregated BESS.

where $\Delta f^{\text {threshold }}$ is chosen as $0.2 \mathrm{~Hz}$ according to Singapore frequency regulation; $B_{f}$ is the frequency bias of the Singapore power system, which is $200 \mathrm{MW} / \mathrm{Hz}$; and $P^{\max }$ is the maximum power rating of the aggregated BESS.

Having defined the frequency bias and the participation factor, the BESS limitations are considered next.

Rule 2: If the SOC is greater than or equal to 0.95, the individual BESS cannot be charged anymore. If SOC is less than or equal to 0.05, the BESS cannot be discharged anymore.

As stated in Rule 2, charging/discharging buffer constraint is set as $5 \%$ of the maximum individual energy of BESS because of safety issues. In hardware tests, real-time SOC is available from the BMS. In simulations, SOC of the individual BESS will be updated by the following equation:

$$
S O C(t)=S O C\left(t_{0}\right)-\frac{\sum_{t=t_{0}}^{t} p(t) \cdot \delta t}{Q}
$$

where $p$ is the power output of the individual BESS; $\delta t$ is the time interval; and $Q$ is the capacity of the individual BESS. 
With operation rules and limitations specified, we should notice that by feeding standard or dynamic regulation commands into the BESS, signal hunting should be avoided. Therefore, a certain dead zone is set in Rule 3 .

Rule 3: If the frequency change is equal to or less than $0.02 \mathrm{~Hz}$, the aggregated BESS will not respond.

Rule 3 imitates the control deadband in the generation plant governor. This prevents the "hunting" of the regulation signal, reducing the influence of random disturbances or metering noises.

\subsection{Sizing Analysis with NERC Criterion}

A larger BESS capacity helps to enhance the stability of the system frequency response notably. However, the total investment is still a major concern. To balance the trade-off between technical and economical issues, a sizing analysis is performed to determine the most cost-effective BESS penetration rate. To ensure the system frequency performance, NERC devised a set of performance criteria called CPS1 and CPS2. The frequency profiles used in the evaluation are obtained from software simulations of the proposed system as in Fig. 3.1 with an aggregated BESS as shown in Fig. 3.4.

\subsubsection{Sizing Analysis Using CPS1 and CPS2}

To get an estimation of the frequency response of the Singapore power system, historical frequency data are used to calibrate the simulation model. The benchmark signals are scaled down from the average NERC standards. Parameters are also downscaled accordingly as listed in Table 3.1.

When conducting the BESS sizing analysis for a control area, the tie-line power fluctuation should be neglected in the planning stage. The sizing analysis starts with an one-area 400-MW system. CPS1 is considered as the primary index, and CPS2 will not be computed since only one control area is involved. 


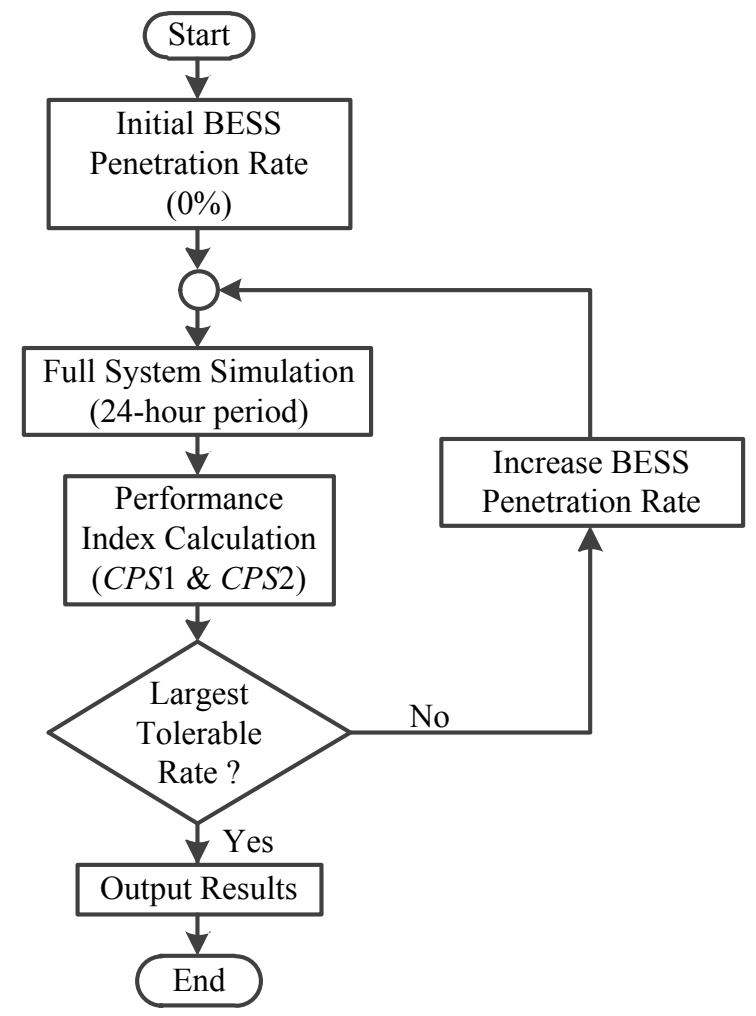

Figure 3.6: Flowchart of BESS penetration rate analysis.

Table 3.1: Parameters for the sizing analysis tests

\begin{tabular}{cc|cc|cc}
\hline Time period & 24 hours & $\mathbf{B}_{\mathbf{f}}$ & $200 \mathrm{MW} / \mathrm{Hz}$ & $\epsilon_{\boldsymbol{1}}$ & $50 \mathrm{mHz}$ \\
\hline $\mathbf{L}_{\mathbf{1 0}}$ & $9.33 \mathrm{MW}$ & $\mathbf{B}_{\mathbf{s}}$ & $400 \mathrm{MW} / \mathrm{Hz}$ & $\epsilon_{\mathbf{1 0}}$ & $20 \mathrm{mHz}$ \\
\hline
\end{tabular}

The BESS penetration rate is initially set at zero. The penetration rate is then increased from $0 \%$ to $50 \%$ at a $5 \%$ step. According to the definitions in the previous subsection, the CPS1 value is a weighted average within the 12 consecutive months. However, since the daily consumption behavior is highly repetitive in Singapore, the 12-month long simulation is not necessary. Here we use a 24 -hour time period as a reasonable simplification. For each penetration scenario, simulation tests are executed using the BESS controller designed previously as in Fig. 3.5. The test data are obtained with 1-s resolution. The sizing analysis process is summarized in the flowchart as in Fig. 3.6 .

The evaluation results of the 24-hour operation are shown in Fig. 3.7. With the increasing of BESS capacity, the one-area $C P S 1$ score is rising, reaching its first peak at a $20 \%$ penetration. However, the system frequency deviation is composed of slow dynamic 


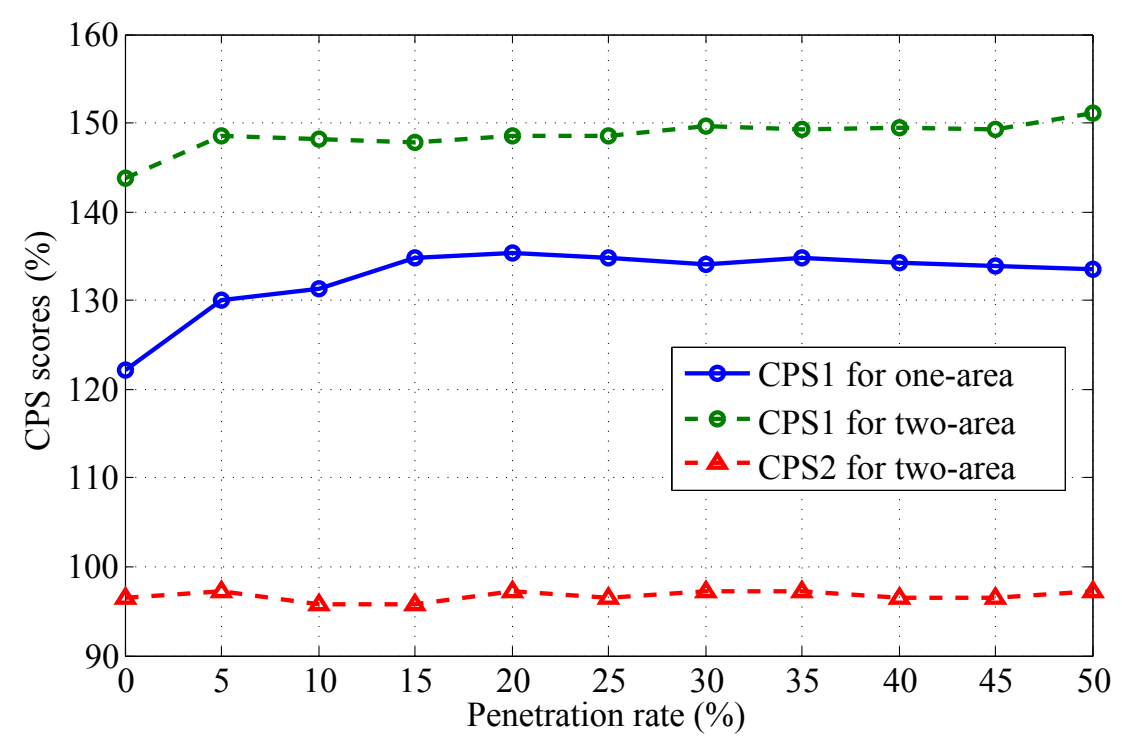

Figure 3.7: CPS scores with different BESS penetration rates.

and fast dynamic components. According to the AGC scheme shown in Fig. 3.3, BESSs are assigned to respond to the fast dynamic component. During the simulation, the regulation share of the power plant is replaced by the BESS capacity step by step. When the regulation share of the BESSs reaches a certain level, the power plant participation share will no longer be enough in dealing with the slow dynamics component. Therefore, after a $20 \%$ penetration rate, the CPS1 score does not increase anymore, and even deteriorates hereafter.

Based on Fig. 3.7, the CPS1 score at 20\% BESS penetration increases less than $1 \%$ compared with that of $15 \%$ BESS penetration. However, the BESS cost increases linearly with its size [17]. Therefore, a $5 \%$ incremental change at 15\% BESS penetration will cause an additional 33\% increase in capital investment while the CPS1 improvement is less than $1 \%$. Considering the huge investment cost and the benefit, it may be more appropriate to set the BESS capacity at a $15 \%$ penetration for a stand-alone system.

Another case study incorporating a two-area power system is simulated. The results are also plotted in Fig. 3.7. Given the help from another area, the BESS penetration rate could be lowered to $5 \%$. The CPS2 score is not apparently affected by the BESS, because it is designed to evaluate the long-term frequency fluctuation and tie-line power.

In the modeling, all the generators in one control area are simplified as one generator. And only one inertia constant (M) is used as the system-wide equivalent value. 
Table 3.2: CPS1 scores for varying $\mathrm{M}$ values

\begin{tabular}{c|c|c|c}
\hline Equivalent M & 8 & 10 & 12 \\
\hline CPS1 score (\%) & 130.2302 & 130.0322 & 129.8959 \\
\hline
\end{tabular}

Table 3.3: Parameters for system frequency simulation

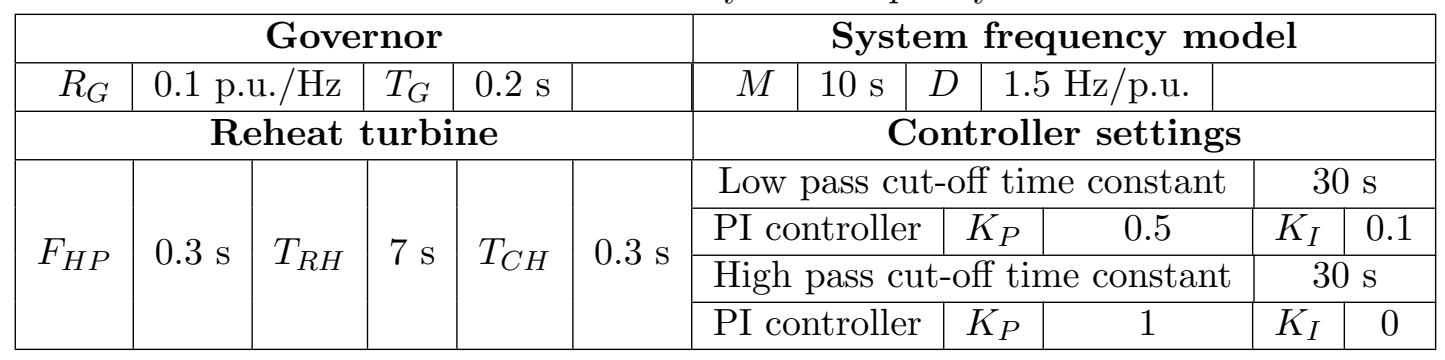

Given multiple machines in the system, the regulation responsibility will be distributed according to their ramp rates and capacity, under the command of the LFC signal. The larger the equivalent inertia, the larger the stored kinetic energy in the rotating mass, which helps to arrest the power mismatch and the frequency change almost instantly.

The results in this section are based on long-term statistical studies on the system frequency performance. After testing with other parameters in the simulation, different $\mathrm{M}$ values (normally ranging from 8 to 12 ) will only have a slight impact on the range of frequency deviation. The system frequency performance is shown in Table 3.2. Therefore, the CPS-based sizing study results will not be influenced by the M value and Fig. 3.7 will display the same trend.

\subsubsection{System Frequency Studies with Suggested BESS Penetration}

System frequency dynamics studies are presented using the simulation model developed in the frequency domain. Scheduled tie-line power exchange is neglected in this demonstration. The peak load of the power system is $400 \mathrm{MW}$, and the load fluctuation is under $20 \mathrm{MW}$, which is $5 \%$ of the peak load. The frequency regulation capacity assigned to the thermal plant is $1 \%$ of the peak load, which is 4 MW. The BESS penetration rate is chosen as $15 \%$ of the regulation requirement which equals to $0.6 \mathrm{MW}$, as suggested in the last Section. All parameters of the simulated system is listed in Table 3.3 .

As shown in Fig. 3.8(a) to Fig. 3.8(d), the system frequency fluctuates due to 


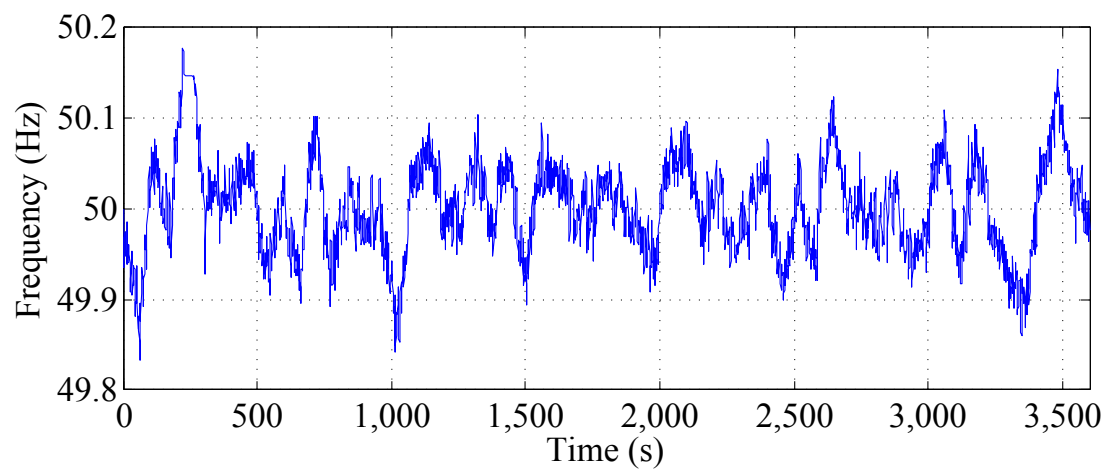

(a) System frequency $(\mathrm{Hz})$

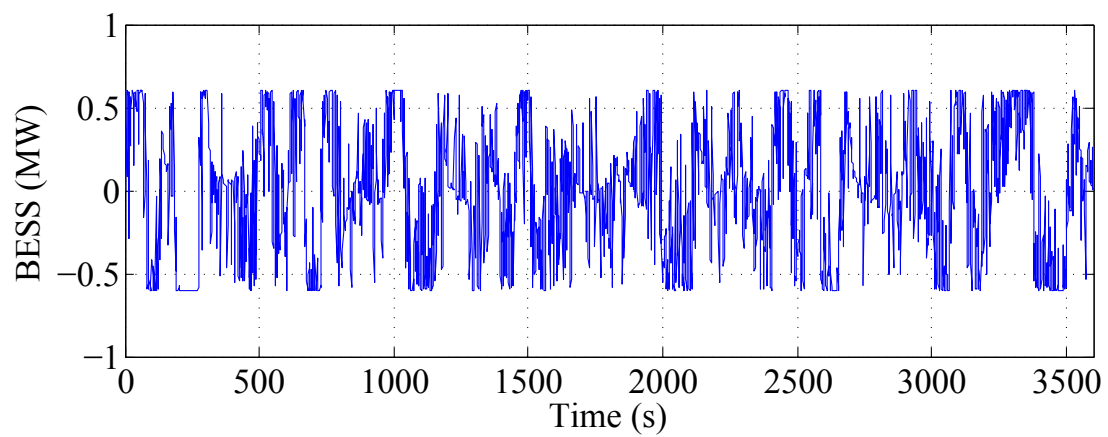

(b) Aggregated BESS output change (MW)

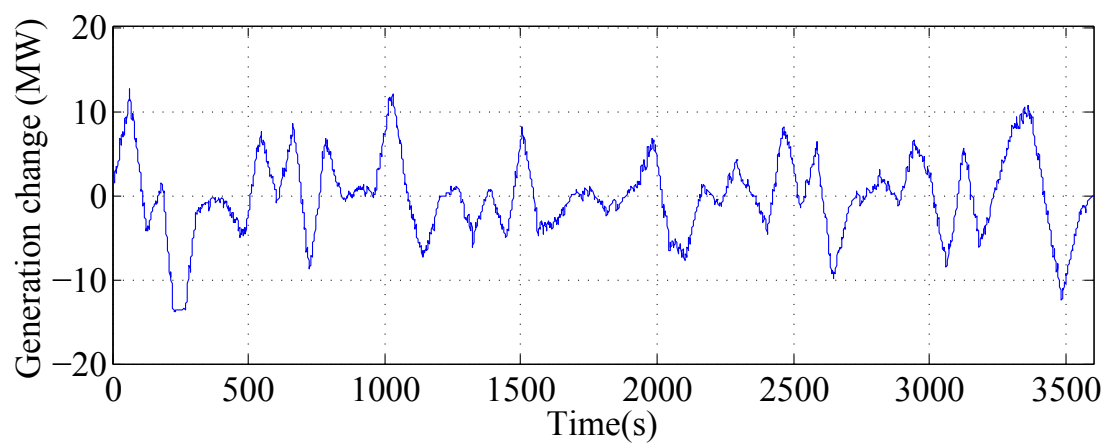

(c) Thermal power plant generation change (MW)

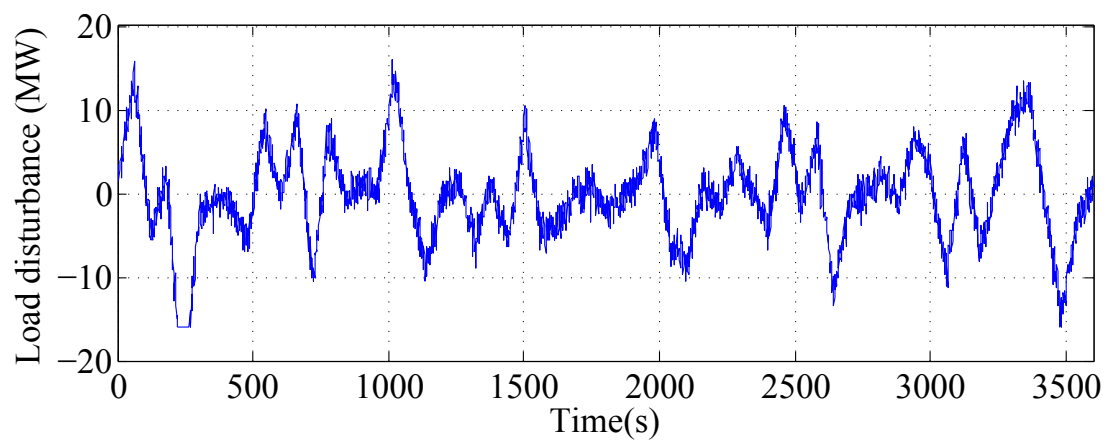

(d) Load disturbance (MW)

Figure 3.8: Results of system frequency studies with a 15\% BESS penetration. 


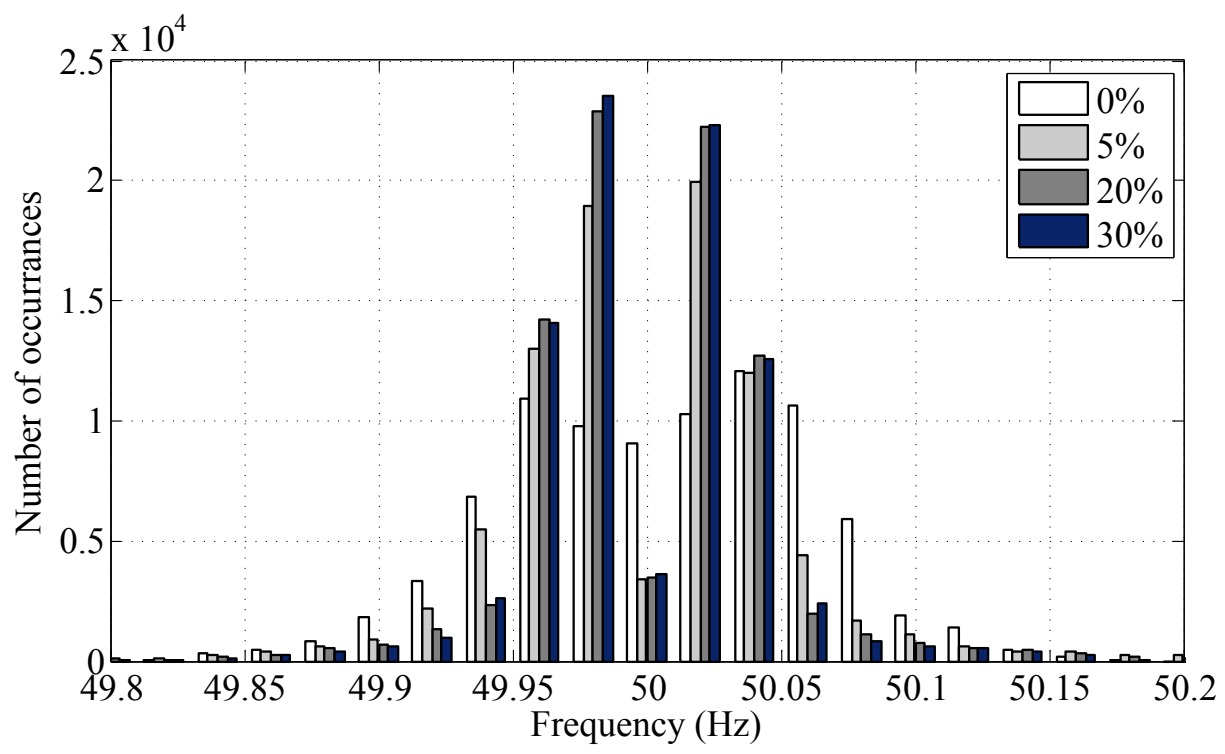

Figure 3.9: Frequency distribution with different BESS penetration rate.

load disturbances. The output of the BESS and that of the thermal power plant vary according to the LFC command. Despite rapid load disturbances, the output profile of the thermal power plant is much smoother than the BESS because of the low-pass filtering in its LFC function to satisfy its ramp rate limitation. In summary, the frequency change is always regulated and controlled to be less than $\pm 0.2 \mathrm{~Hz}$ using both the BESS and the thermal plant.

The frequency profiles for different BESS penetration rates of $0 \%, 5 \%, 20 \%$ and $30 \%$ are compared. The frequency deviation distribution characteristics are studied. According to the histogram in Fig. 3.9, large frequency fluctuations happen less with BESS penetration. However, the frequency distribution characteristics of 30\% BESS penetration rate is similar to those of $20 \%$, which agrees with the CPS1 score plotted in Fig. 3.7.

PV generation is intermittent and can cause rapid disturbances. Therefore, the impact of the PV generation on the CPS1 score is also studied with five days PV generation data recorded in NTU. As shown in Fig. 3.10, system frequency performance drops with the increase of the PV penetration rate. Given the suggested BESS regulation capacity for the tested system, the PV penetration rate is limited to a certain percentage, beyond which the system $C P S 1$ score will drop below the minimum requirement. Therefore, if the power grid requires more PV capacity, larger BESS systems need to be installed, 


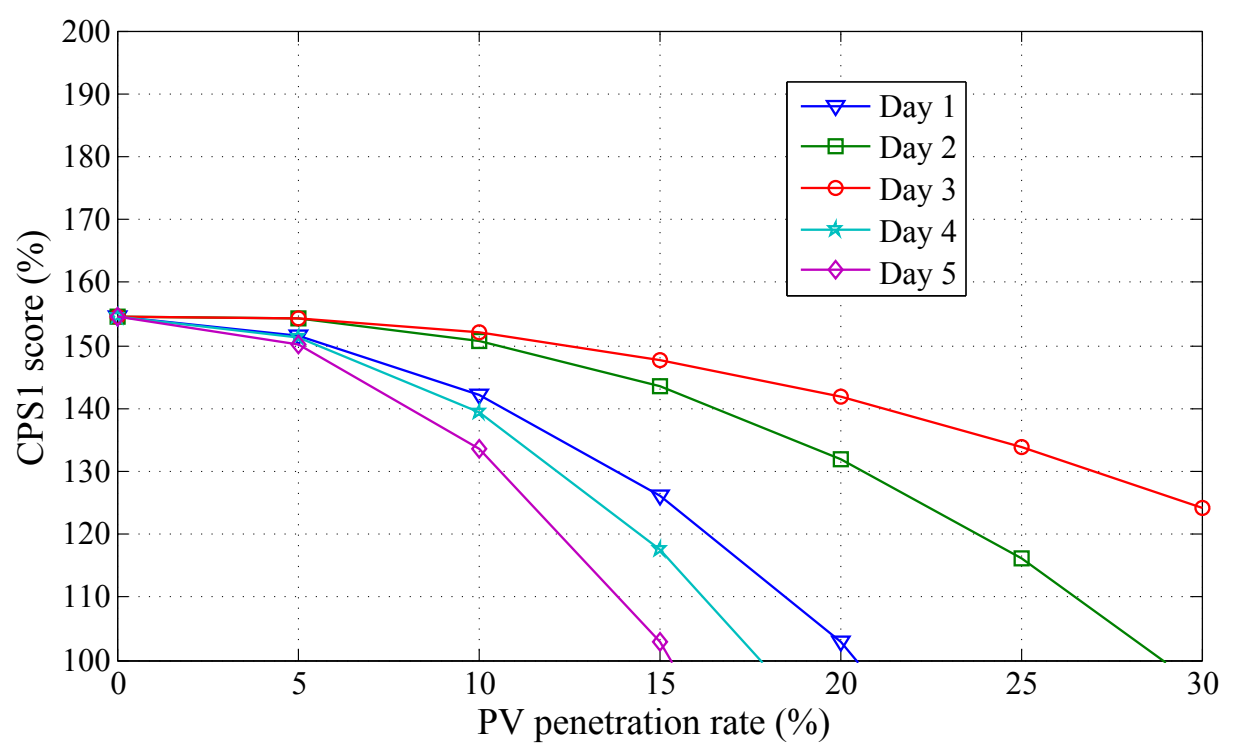

Figure 3.10: CPS1 with different PV penetration rate.

and more advanced control strategy could be utilized.

\subsection{BESS Management and Performance Studies}

\subsubsection{BESS Effectiveness Analysis}

In order to evaluate the effectiveness of each power source in responding to regulation signals, specific contributions from the thermal power plant and the BESS are quantified. The concept of the net $A C E$ corrected energy ( $\left.A C E^{n e t}\right)$ is introduced [109]. The $A C E$ corrected energy $\left(A C E^{c o r}\right)$ is defined as the area where the output of the power source follows the negative value of $A C E$. The against- $A C E$ energy $\left(A C E^{a g s t}\right)$ is defined as the area where the output of the power source follows $A C E$. The $A C E^{\text {net }}$ is defined as the difference between the $A C E^{c o r}$ and the $A C E^{a g s t}$ as in (3.9). The total power output $A C E^{\text {total }}$ resulting from following the ACE signal is the summation of $A C E^{c o r}$ and $A C E^{a g s t}$ as shown in (3.10).

$$
\begin{aligned}
& A C E^{\text {net }}=A C E^{c o r}-A C E^{a g s t} \\
& A C E^{\text {total }}=A C E^{c o r}+A C E^{a g s t}
\end{aligned}
$$




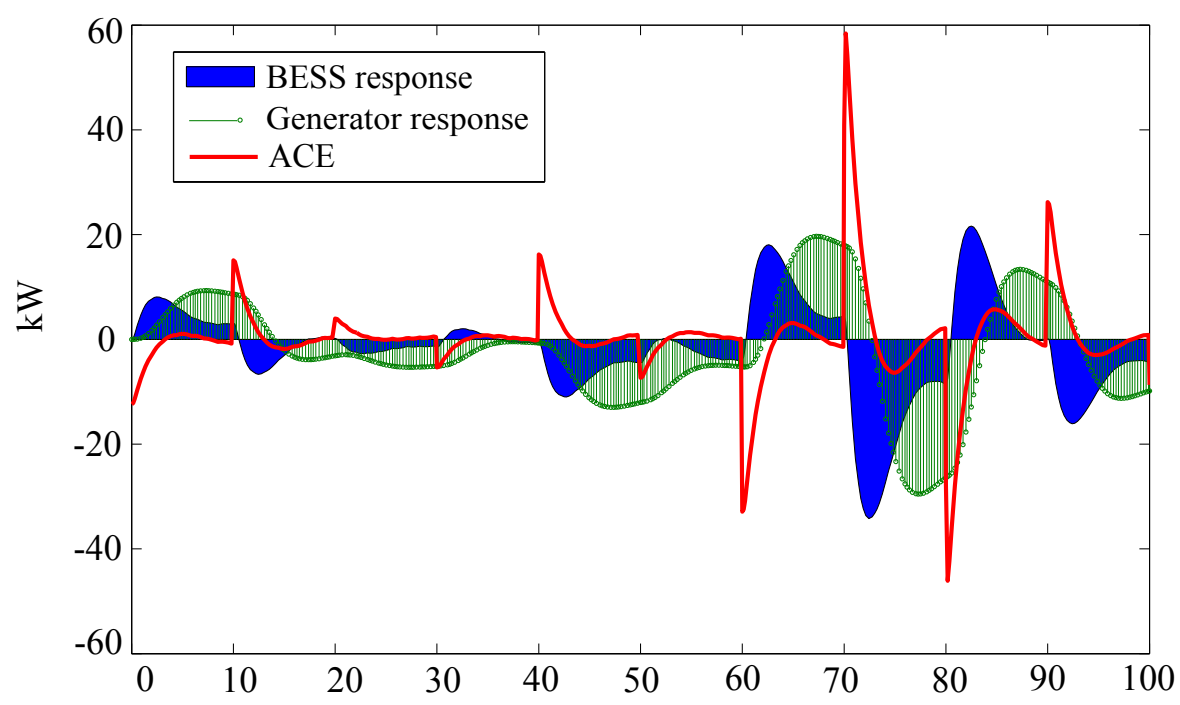

Time (s)

Figure 3.11: Comparison of battery system and slow generator regulation.

Figure 3.11 shows a comparison of the amount of energy delivered by BESS and that of a generator. The red curve is the amount of energy actually required to neutralize the load imbalance. The blue shaded area, which indicates BESS output, follows the red curve tightly but in the opposite direction, while the green shaded area represents the generator energy output which lags behind, and sometimes even acts against $A C E$. It is easy to see that the BESS is much more efficient for short transient imbalances during the $100 \mathrm{~s}$ of the simulation period.

Table 3.4 shows the numerical results of the above simulation study. The $A C E^{c o r}$ supported by the BESS and generator is $0.1352 \mathrm{kWh}$ and $0.1338 \mathrm{kWh}$ respectively. These two values are very close to each other. The generator's $A C E^{\text {total }}$ is $0.2496 \mathrm{kWh}$, and that of the BESS is $0.1612 \mathrm{kWh}$. This is because of their different participation factors. The thermal power plant provides two times more regulation capacity than the BESS. Besides, the time delay resulted from the power plant's governor and turbine dynamics as shown in Fig. 3.11 is also noticeable in the $A C E^{\text {agst }}$ values. The $A C E^{\text {agst }}$ supplied by the BESS and generator is $0.0260 \mathrm{kWh}$ and $0.1158 \mathrm{kWh}$, taking up $16.1 \%$ and $46.4 \%$ of their total energy outputs respectively.

However, the $A C E^{\text {net }}$ supported by the BESS is $0.1092 \mathrm{kWh}$. This value is six times more than that supported by the generator, which is $0.0180 \mathrm{kWh}$. It implies that fast 
Table 3.4: Comparison of frequency regulation effective level

\begin{tabular}{ccc}
\hline & BESS & Generator \\
\hline ACE corrected energy & $0.1352 \mathrm{kWh}$ & $0.1338 \mathrm{kWh}$ \\
Against-ACE energy & $0.0260 \mathrm{kWh}$ & $0.1158 \mathrm{kWh}$ \\
Total ACE energy & $0.1612 \mathrm{kWh}$ & $0.2496 \mathrm{kWh}$ \\
Net ACE corrected energy & $0.1092 \mathrm{kWh}$ & $0.0180 \mathrm{kWh}$ \\
\hline
\end{tabular}

responding units are much more effective in following $A C E$, and hence are beneficial in reducing the system regulation reserve capacity.

A more in-depth effectiveness analysis of BESS is conducted with different levels of rapid disturbance power. The results are shown in Fig. 3.12. An effective index EI is defined in (3.11):

$$
E I=\frac{A C E_{B E S S}^{n e t}}{A C E_{t h}^{n e t}}
$$

The $E I$ values are plotted in Fig. 3.12. The effectiveness index keeps growing with the increasing disturbance power. The rapid disturbance power is limited within $[0.5 \%, 7.0 \%]$ of the peak demand since $A C E_{t h}^{n e t}$ drops below zero after a $7.0 \%$ disturbance. This means that the traditional power plant is unable to cope with large rapid disturbances which will come with future high PV penetration. Given rapid PV fluctuations, the thermal power plant can no longer help in sustaining system frequency while $A C E_{B E S S}^{\text {net }}$ remains high.

Therefore, when participating in frequency regulation, BESS should be rewarded with a higher price for their high effectiveness. In the United States, the fast regulation price triples the regular price [41] while a similar pricing scheme for BESS in Singapore is currently under discussion by the Energy Market Authority.

\subsubsection{Frequency Regulation Participation}

A distributed small-scale BESS is tested as a part of an aggregated BESS instead of a large-scale BESS. It is a reasonable simplification based on the VPP concept introduced. Specifically, we study a single branch of the aggregated BESS as in Fig. 3.4 and Fig. 


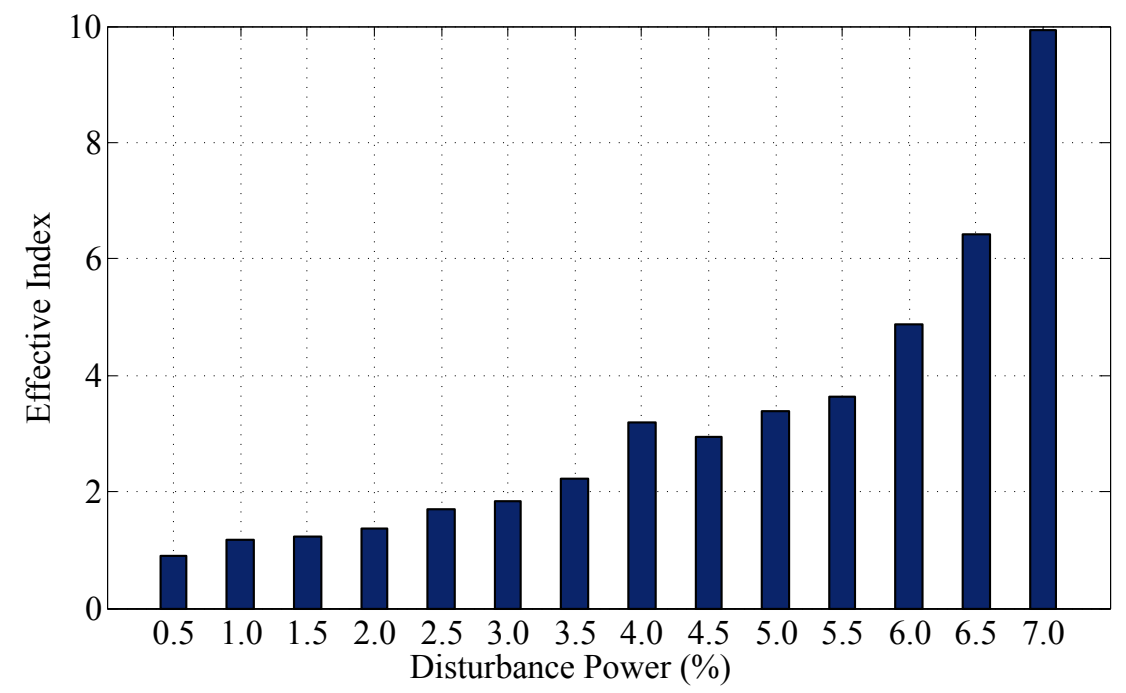

Figure 3.12: Effectiveness study results with different disturbance power.

3.5. Therefore, the BESS Aggregation Center is similarly simplified to a distributed local BESS controller. The proposed controller is tested in simulations based on real metered data. The battery power rating is $10 \mathrm{~kW}$. The charging/discharging buffer is set as $5 \%$ of the total BESS energy. The tie-line power flow is very weak between Singapore power grid and the Malaysian grid, thus $\Delta P_{t i e}$ is ignored in the real system testing. The participation factor $\rho$ can be calculated as 0.00025 according to (3.7), where $\Delta P_{\text {tie }}$ is set as zero.

The real time frequency data were measured by a smart meter ION 7550 installed in the microgrid of Clean Energy Research Laboratory (CERL), Nanyang Technological University. It transmits the dynamic frequency data to the LabView database through TCP/IP communication. The data logging and supervisory control module of LabView is used. This module includes tools for logging data to a networked historical database, as well as real-time and historical trending, managing alarms and events, and networking LabView real-time targets and Modbus devices.

Real-time frequency data in November 2012 is recorded and plotted in Fig. 3.13, and used in the software simulation. The initial SOC of BESS is set at 0.5. The sample time is $1 \mathrm{~s}$. After the simulation, the SOC of the BESS is recorded in Fig. 3.14.

As shown in Fig. 3.14, the SOC of BESS is kept between 0.05 and 0.95. When the SOC is less than or equal to 0.05, the BESS will not accept any discharging command except for charging commands. When the SOC is greater than or equal to 0.95 , the 


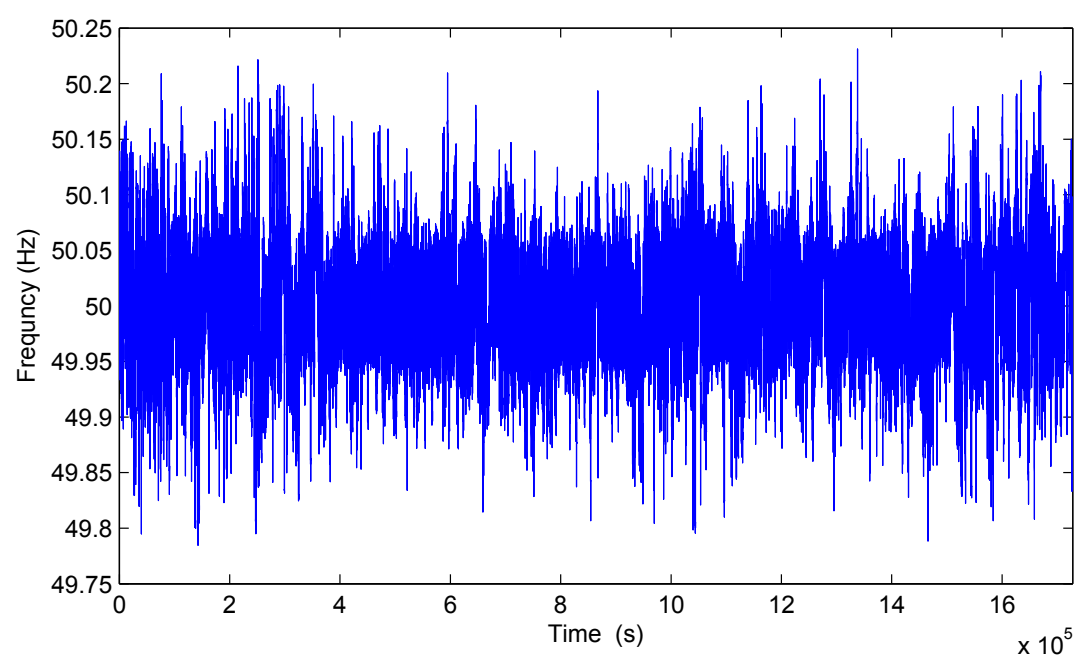

Figure 3.13: Singapore frequency data in November of 2012.

BESS will not accept any charging commands except for discharging commands.

The overall output power of BESS is shown with two enlarged windows in Fig. 3.15. The BESS power is limited within its rated power $10 \mathrm{~kW}$. When the frequency change is equal to or greater than $0.2 \mathrm{~Hz}$, the output power of BESS will be set at the maximum value of $10 \mathrm{~kW}$. From the partial enlarged view of Fig. 3.15, the BESS cannot be discharged when its SOC drops to the lower limit. As one can see in the enlarged window, the BESS output power is locked at zero during SOC depletion. Conversely, it cannot be charged any more when the BESS SOC rises to the upper limit. Therefore, in the second window, the BESS output power returns to zero during SOC saturations.

The periods during which the battery output is locked at zero are defined as nonresponse time. It includes the time the BESS cannot be discharged when there is no energy left inside the battery, as well as the time it cannot be charged when the battery energy is in its full capacity. Using Fig. 3.15, the total non-response time of the BESS is computed. The total non-response time of BESS is $140,863 \mathrm{~s}$ across the span of 20 days in November 2012. The percentage of the total non-response time period can be calculated as $8.15 \%$.

Meanwhile, to further assess the battery performance, the equivalent discharge cycle number is used as another performance criterion here. The equivalent discharge cycles can be obtained by using the total discharge energy divided by the battery capacity. 


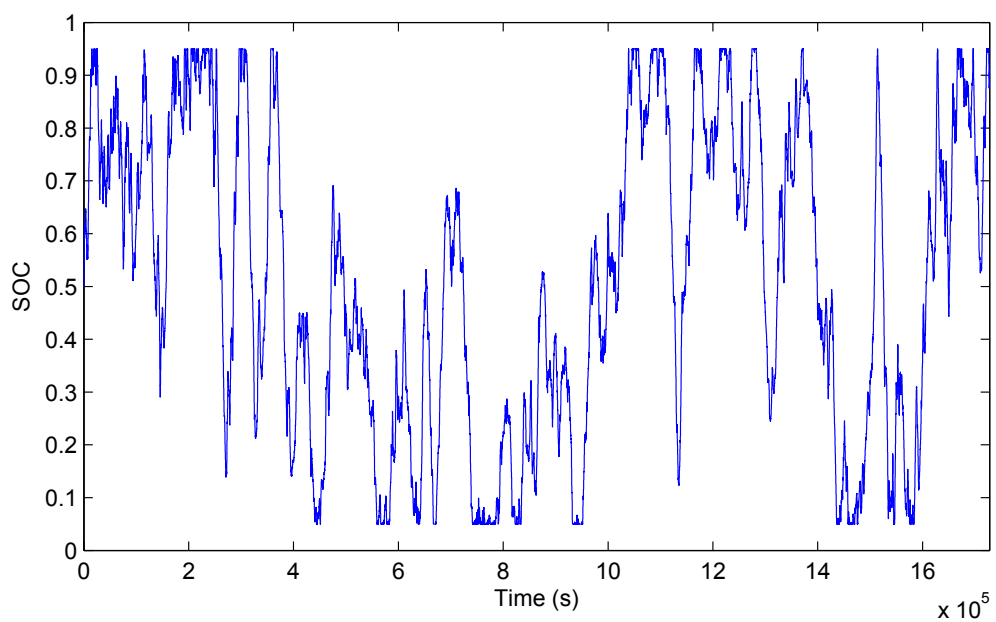

Figure 3.14: SOC of storage battery system.

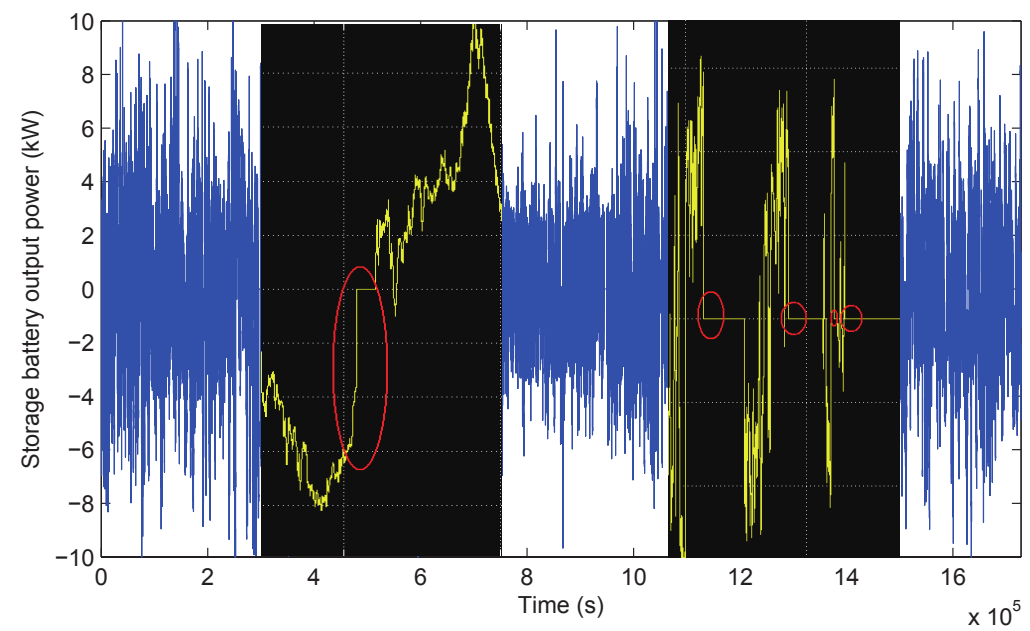

Figure 3.15: Partial enlarged view of power output of storage battery system.

For example, the total discharge energy based on Fig. 3.15 is $502 \mathrm{kWh}$. The battery capacity is $15 \mathrm{kWh}$. Therefore, the total discharge cycle is calculated as 33.5. Similarly, the total charge energy is $508 \mathrm{kWh}$ and the equivalent charge cycle is 33.9 . If we take the average of 33.5 and 33.9 , the equivalent usage life cycles can be obtained as 33.7.

Table 3.5 shows the comparison of the BESS performance for different time periods. Three time periods are chosen in the comparison. The equivalent usage life cycles of the BESS for one week, 20 days and 40 days are 11.8, 33.7 and 63.35 respectively and their corresponding percentages of the total non-response time are $8.03 \%, 8.15 \%$ and $8.47 \%$. One can deduce that the equivalent usage life cycles has a linear relationship 
Table 3.5: Comparison of usage life cycle and total non-response time

\begin{tabular}{ccc}
\hline & $\begin{array}{c}\text { Equivalent usage } \\
\text { life cycles }\end{array}$ & $\begin{array}{c}\text { Percentage of total } \\
\text { non-response time }\end{array}$ \\
\hline $\begin{array}{c}\text { One week } \\
\text { (Jan 2013) }\end{array}$ & 11.8 & $8.03 \%$ \\
\hline $\begin{array}{c}20 \text { days } \\
\text { (Nov 2012) }\end{array}$ & 33.7 & $8.15 \%$ \\
\hline $\begin{array}{c}40 \text { days } \\
(\text { Nov 2012 - Dec 2012) }\end{array}$ & 63.35 & $8.47 \%$ \\
\hline
\end{tabular}

with the time period. However, the percentages of the total non-response time are all around $8.25 \%$, which is not affected by the time period. Only when the aggregated BESS reaches a comparatively large capacity, the depletion and saturation time can be reduced, meaning that the percentage of the total non-response time will drop evidently [19].

\subsection{Summary}

This chapter analyzed the performance of the aggregated BESS participating in frequency regulation. Studies include frequency regulation performance tests and sizing analysis. Software simulations are conducted.

The recommended BESS penetration rate in the power system is analyzed using an iterative approach according to the NERC performance indexes. Taking the investment cost into consideration, a $5 \%$ to $15 \%$ BESS penetration rate is recommended for the test system. The analysis method can also be applied to other systems. By incorporating the recommended BESS in the power grid, the system frequency deviation distribution is improved based on the simulation results.

In order to estimate the impact of the PV generation on the CPS1 score, simulations with different PV penetration rates and the suggested BESS penetration rate are conducted. With a certain PV penetration rate, the CPS1 score will decrease below the minimum requirement. Therefore, BESS utilization involving PV characteristics is to be addressed in the future work.

It is shown that BESS is fast-responsive, and is more effective than traditional fuel- 
based generators in driving the ACE to zero. A comprehensive effectiveness study is done with different levels of disturbance power.

Aggregation of distributed BESSs is recommended to expand the grid BESS capacity. The proposed rule-based controller is suitable for aggregating large amount of dispersed BESSs. The only data required from the centralized EMS is the frequency data. And the BESS Aggregation Center distributes the control signal to the BESSs involved in its control area. When participating in frequency regulation, the BESS operates in the desired SOC zones for most of the time. However, a non-response time is observed. The non-response time remains similar regardless of the test duration. 


\section{Chapter 4}

\section{Hierarchical MPC-based EMS for a VPP with BESSs}

In Chapter 3, the system performance with aggregated BESSs is analyzed under the current frequency regulation scheme. In this chapter, a model-based frequency controller will be developed for aggregated BESSs and other resources, to make good use of BESSs and to coordinate various resources. This will further improve the overall system dynamics.

\subsection{Introduction}

With the ambition to reduce greenhouse gas emission and incorporate more renewable energy sources into the grid, PV installation capacity has been expanding rapidly. Recently, lots of PV panels are installed on the rooftop or the exterior surface of residential housing units or commercial buildings. This means PV generation units are increasingly decentralized and electrically near the loads of the distribution network [110]. Therefore, regulation and stabilization becomes a critical issue due to their intermittency and uncertainty nature. In order to fully dispatch their capacities, PV generation systems are often accompanied with BESSs [53] or clustered with other energy sources and loads to form microgrids or VPPs [111].

During power system operation, system frequency directly reflects whether active 
power is balanced or not. Systems with large PV penetration may encounter even larger and more rapid frequency oscillations, resulting from the decreased system inertia. The time frames of power dispatch and frequency stabilization are different, ranging from seconds to minutes. For this reason, a hierarchical control structure is highly recommended for VPP operators to combine these two tasks into a single multi-level controller. The goal is to imitate the dispatch and regulation behaviors of conventional power plants, which participate in an open market to sell extra energy and provide ancillary services, such as demand response and spinning reserve.

In this chapter, the main contribution is the design of a centralized hierarchical hybrid MPC controller to conduct area frequency control and to achieve VPP economic dispatch on two-level timescales with different control targets defined at each level. System frequency dynamics, physical constraints and SOC constraints of the storage system are considered in the calculation framework. With the proposed EMS, VPP is able to control the system frequency in addition to manage normal BESS charging/discharging requirements. Besides, PV converter maneuvering is reduced by the Lasso MPC controller in the lower level [112]. The proposed control scheme is demonstrated in a simple one-area system with a lumped model VPP and a traditional thermal power plant.

The rest of the chapter is organized as follows: Section 4.2 describes the hierarchical hybrid MPC controller. Optimal problem settings are discussed in detail. Section 4.3 describes the test system configuration and modeling. Section 4.4 records and analyzes the numerical simulation results. The research contents are summarized in Section 4.5.

\subsection{Hierarchical Control Mechanism}

The proposed controller could be located in the control center of a VPP, where the controller has access to prediction and operation data from different energy aggregators: the load aggregator, BESS aggregator, PV aggregator and a combined-heat-and-power (CHP) generation plant. The VPP controller can communicate with generation and transmission companies. The system structure is summarized in Fig. 4.1.

Within the proposed EMS, a two-level hierarchical control structure as in Fig. 4.2 is proposed. The primary control, which imitates the speed governor in a conventional 


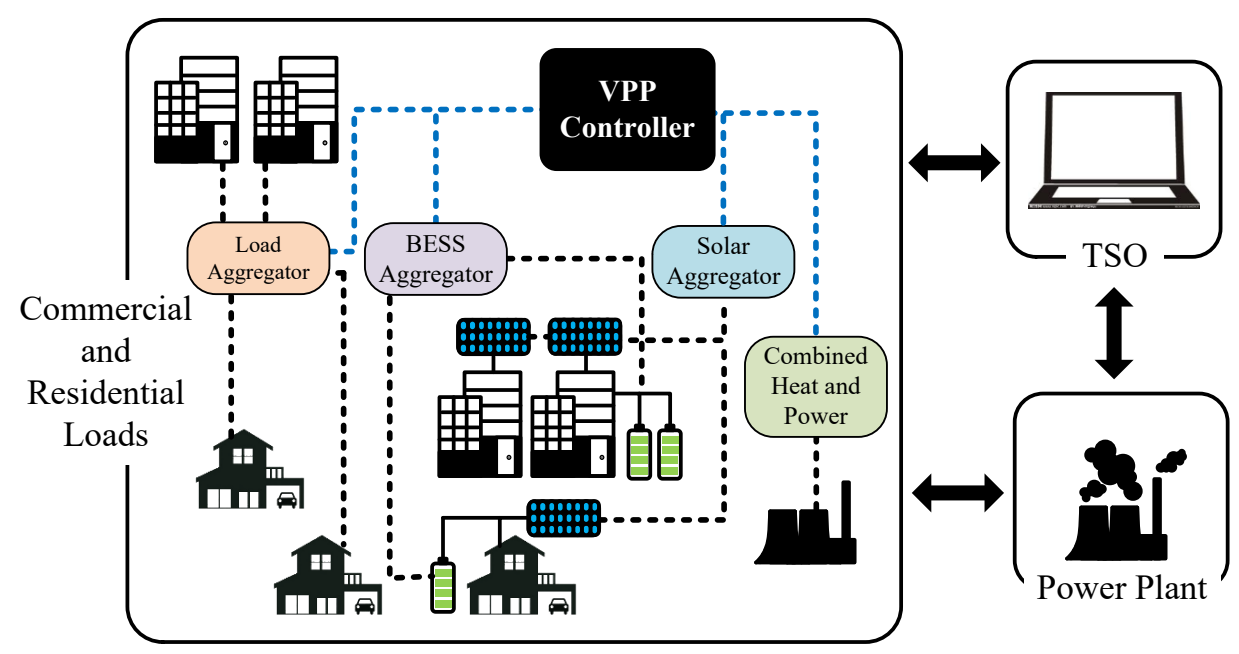

Figure 4.1: Overall power system structure with VPP.

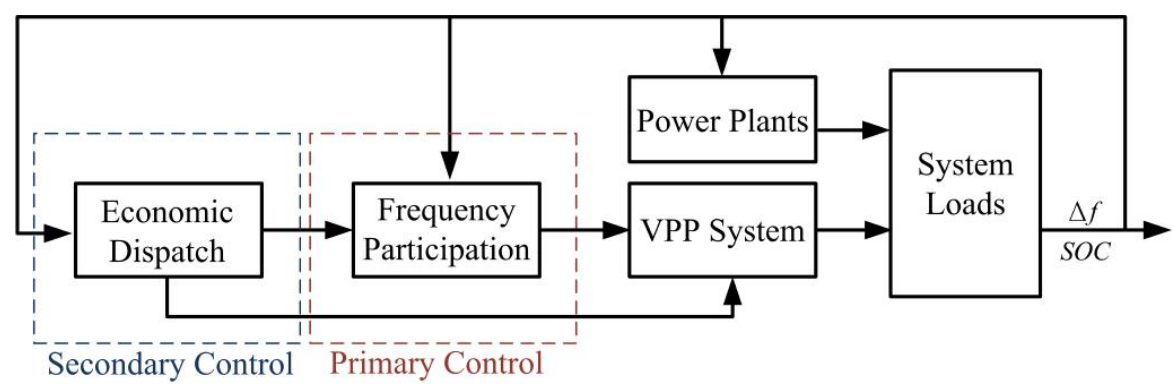

Figure 4.2: Structure of a hierarchical MPC controller.

power plant, is designed to eliminate the frequency deviation produced by the power mismatch between power generation and load consumption. Its main control objective is to optimize the frequency trajectory, and at the same time, to allocate power demand between slow traditional units and fast responding battery systems. Beyond its scope of frequency stabilization in short terms of several seconds, a long-term set-point adjustment in every hour is accomplished in the secondary control, where an economic oriented MPC is applied to dispatch the power from the PV and the BESS within the VPP. The objective is to coordinate PV and BESS for the maximization of economic benefits.

During real-time operations, the secondary control executes long-term optimal power dispatch for a 24-hour interval, providing set points for units involved. While acting at a shorter time interval in seconds, the primary level takes the set point references as inputs, and controls system frequency at the same time. Therefore, the final power output from the BESS is not only dependent on the slow varying component from the 
secondary control, but also varies according to the faster varying component from the primary control. The proposed control scheme gathers information regarding the grid performances as its feedback inputs.

\subsubsection{Primary Control: Lasso MPC}

The primary control MPC aims to allocate power demand among the PV converter, CHP, and the BESSs in the VPP to achieve power balance and load frequency control. As frequency signals are always fluctuating and may have rapid and drastic variations due to the intermittency of the solar energy, frequent adjustment of the set point could happen from time to time. However, for the CHP generation plant, over-actuation will cause wear and tear of mechanical components, resulting in maneuvering and reversal costs and extra operation and maintenance expenses [3]. Therefore, the control strategy is designed to limit CHP movement to the least, that is, the CHP only participates when it is really necessary while BESS takes over most fluctuations. A Lasso MPC scheme proposed by [112] is highly suitable for this work. In the objective function, both quadratic and linear penalties are adopted to optimize frequency trajectory with least control effort as well as to exert a high cost to actuate PV converter set point. The control problem is presented as (4.1)-(4.2).

The primary level objective function is:

$$
J_{1}=\sum_{k=0}^{N} x^{T}(k) \cdot Q \cdot x(k)+u^{T}(k) \cdot P \cdot u(k)+G \cdot\left\|u_{C H P}\right\|_{1}
$$

where $x(k)$ represents the predicted frequency deviation profile. Decision variable $u(k)=$

$\left[\begin{array}{ll}u_{C H P} & u_{B E S S}\end{array}\right]^{T}$, with $u_{C H P}$ and $u_{B E S S}$ represent CHP and BESS commands respectively. $Q$ and $P$ are weighting matrices, and $G$ is a weighting scalar defined to adjust the actuating price of the CHP. Values of the Weighting factors require tunings to achieve the a desired control performance.

The frequency prediction mechanism is a classical frequency response model. The overall frequency dynamic could be expressed as: 


$$
2 \cdot H(\dot{\Delta f})=\Delta P_{P V}+\Delta P_{C H P}+\Delta P_{B E S S}-\Delta P_{L}-D \cdot \Delta f
$$

For the constraints, frequency deviation limitation and capacity constraints from the CHP power plant and the BESS are all considered.

\subsubsection{Secondary Control: Economic Oriented MPC}

For market clearing purpose, economic dispatch operates every hour in the secondary control with a long prediction horizon, which is 24 hours in our case. A more frequent dispatch is also applicable, depending on the forecasting accuracy of the solar power output and the load demand curve. The problem formulation is described below.

The secondary level objective function in (4.3) uses the energy cost to minimise power purchase. The electricity tariff $c_{\text {grid }}$ represents electricity prices based on Singapore timeof-use rates. In other cases with micro turbine generators using fuels as energy source, $\mathrm{CO}_{2}$ emission could also be introduced into the objective function to reduce the carbon footprint.

$$
J_{2}=\min \sum_{t=1}^{24} P_{\text {grid }}(t) \cdot \Delta t \cdot c_{\text {grid }}(t)
$$

where $P_{\text {grid }}$ represents the power exchange with the utility grid.

Active power balance serves as the equality constraint. In (4.4), $P_{\text {solar }}$ and $P_{\text {demand }}$ are predicted PV outputs and load demand. In this research, prediction mechanism is not discussed. Simulation results are calculated using real load profile and real weather data as perfect prediction data.

$$
P_{\text {grid }}+P_{\text {solar }}+P_{B E S S}+P_{C H P}=\text { Pdemand }
$$

The SOC value of the BESS within the VPP is utilized as inequality constraints. Though several BESSs could be coordinated in a VPP, we use a lumped parameter model to represent its status for simplification. The total SOC is constrained between 


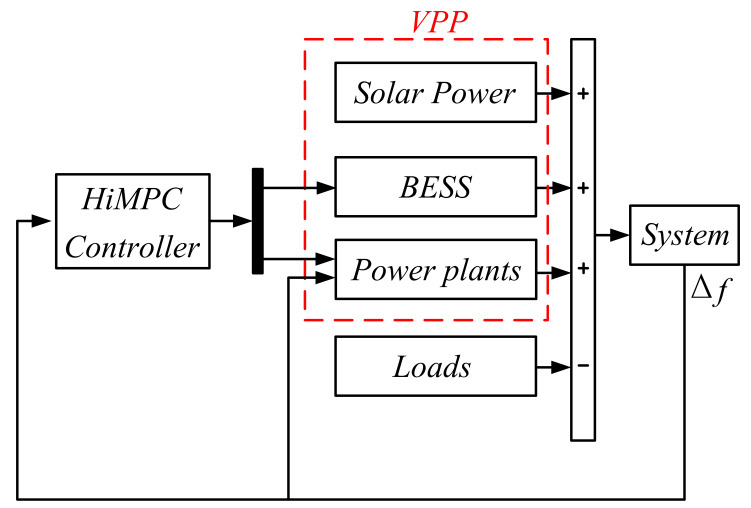

Figure 4.3: Test system configuration.

$20 \%$ and $80 \%$ to limit the battery in its linear operation zone, and provide proper buffer capacity for unexpected events that might require additional charging or discharging.

$$
0.2 \leq S O C(t) \leq 0.8
$$

The SOC value could be predicted by a simplified formulation dependent on the Coulomb counting method, neglecting various types of battery charging/discharging characteristics.

$$
S O C(t+\Delta t)=S O C(t)-\frac{P_{B E S S} \cdot \Delta t}{E_{B E S S}}
$$

where $E_{B E S S}$ is the rated battery capacity.

\subsection{Test System Structure and Modeling}

The VPP system under test is composed of a CHP power plant, a PV energy system, BESS and uncontrollable loads. The overall system configuration is illustrated in Fig. 4.3 .

\subsubsection{Lumped Solar System Model}

The most commonly used characteristic of PV output power is 


$$
P_{P V}=\eta S \Phi 1-0.005\left(T_{a}+25\right)
$$

where $\eta$ is the energy conversion efficiency of the PV panels; $S$ is the measured area of the PV panels; $\Phi$ is the solar radiation value; and $T_{a}$ is the ambient temperature in degree Celsius. Normally, the conversion efficiency and the panel measured area are considered constant, and $T_{a}$ is set as $25 \mathrm{C}$. Therefore, the PV output power could be regarded as linearly varying with solar radiation only. Accordingly, its transfer function could be expressed as:

$$
G_{P V}=\frac{K_{P V}}{1+s T_{P V}}
$$

\subsubsection{Lumped BESS Model}

BESSs are able to ramp up and down very quickly. Therefore, BESSs are regarded as an ideal power sources in this chapter. A lumped BESS is modeled as a first-order transfer function $G_{B E S S}$ in (4.9). The output gain $K_{B E S S}$ is defined according to the size of the BESSs. The time constant $T_{B E S S}$ represents the time delay in communication and control.

$$
G_{B E S S}=\frac{K_{B E S S}}{1+s T_{B E S S}}
$$

\subsubsection{CHP Power Plant Model}

The power plant is represented by a traditional non-reheat thermal generation unit in the simulation. The whole model covers the dynamics of the governor, the turbine and the generator as illustrated in Fig. 4.4. 


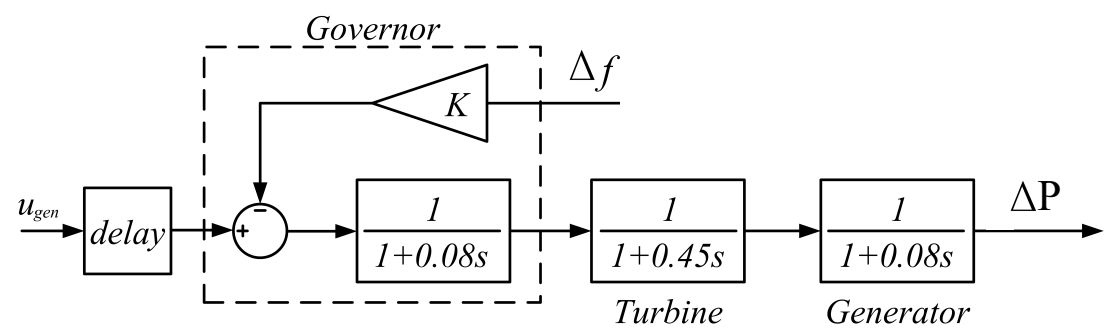

Figure 4.4: Non-reheat thermal power plant model.

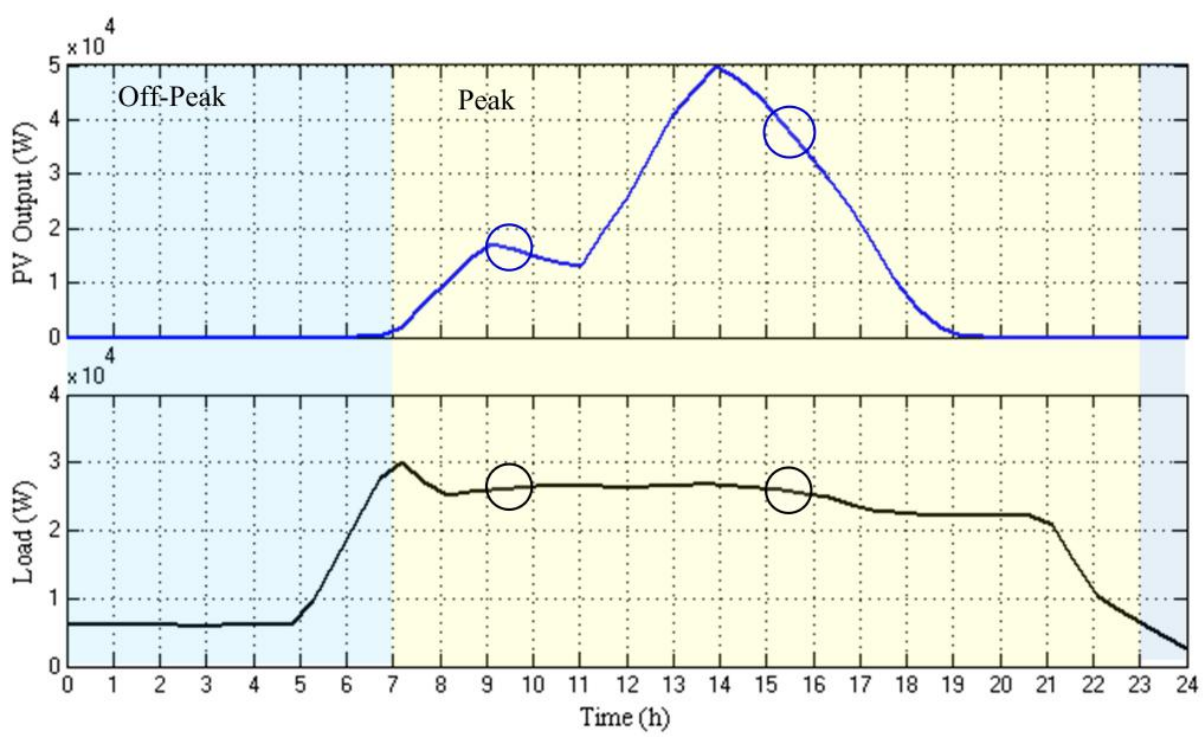

Figure 4.5: Hourly PV output and load curve.

\subsection{Simulation Results}

\subsubsection{Simulation Environment}

A numerical study of the proposed controller is tested with real grid data in this section. The simulation includes real temperature, solar insolation data and load data per minute. The PV output data are calculated from (4.7). And the hourly average is used as the prediction information. The hourly PV output and load curve are shown in Fig. 4.5. Peak average PV generation is $50604.8 \mathrm{~kW}$. The peak average load value is $31277.3 \mathrm{~kW}$. System base is chosen as $50 \mathrm{MW}$. Total BESS capacity is set as $80 \mathrm{kWh}$. Two circled periods are tested during simulation. 


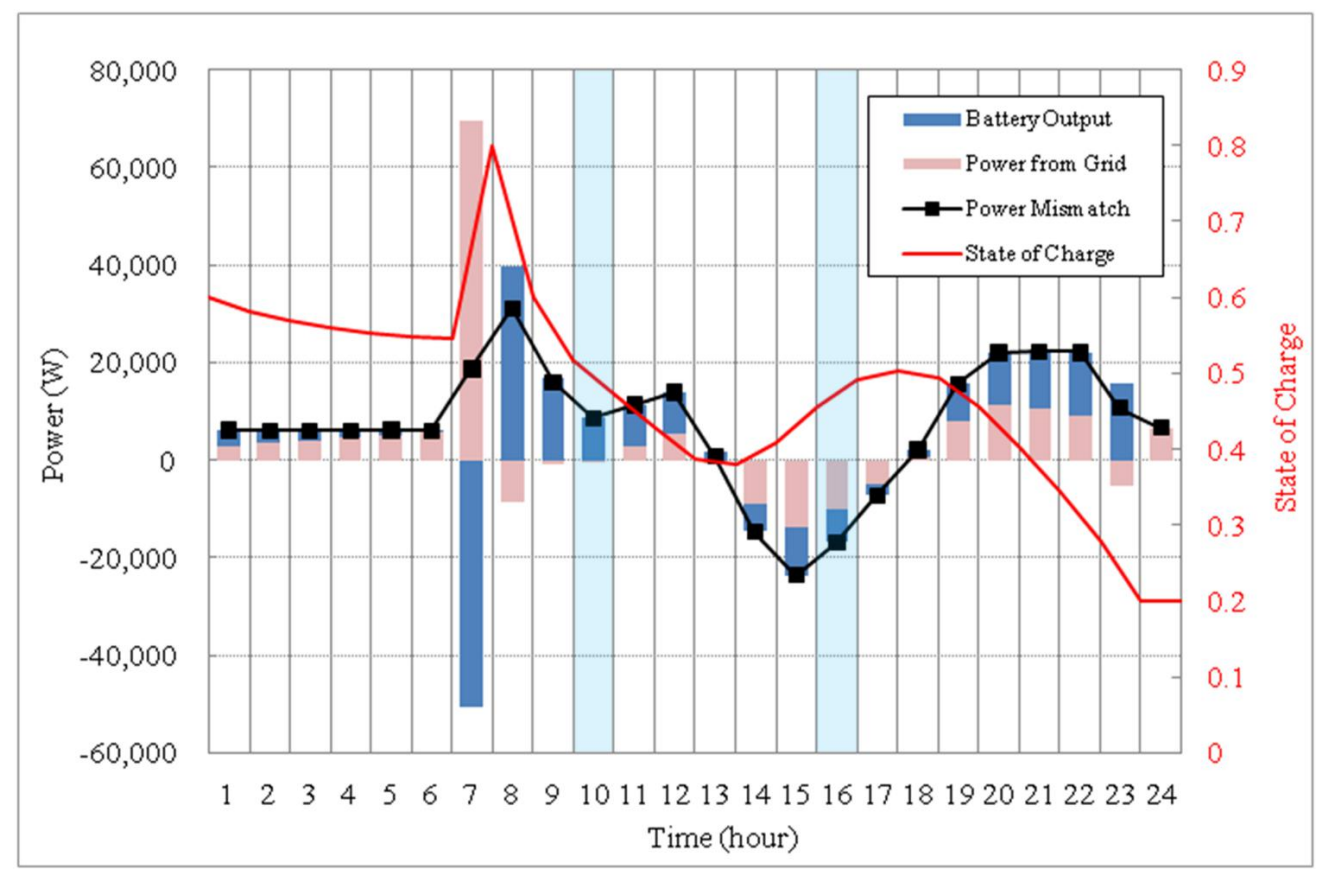

Figure 4.6: Dispatch strategy at the first hour of the day.

\subsubsection{Economic Dispatch}

The time-of-use electricity price from the website of Singapore Power Services is used in the economic dispatch, which is $25 \mathrm{c} / \mathrm{kWh}$ during peak hours (7:00 am to 11:00 $\mathrm{pm})$ and $15 \mathrm{c} / \mathrm{kWh}$ during off-peak hours (11:00 pm to 7:00 am). Optimization results shown in Fig. 4.6 are the dispatch strategy calculated at 12:00 am. The marked black line represents the power mismatch in the system, equivalent to load demand minus PV generation. The red curve represents the predicted state of charge. The pink and blue histograms show the optimal amount of power from grid and from the battery system. From the first hour to the seventh hour, when there is no solar insolation, the demand is met using the power from grid and the BESS. The power from the BESS is gradually decreasing to maintain a certain SOC level. In order to support the load consumption during day-time when the electricity tariff is much higher, in the last hour of the off-peak hours, the battery is charged a great amount of energy from the grid. The charged energy supports the demand for most of the day, especially when the PV power is insufficient. In the afternoon, from 1 p.m. to 4 p.m., when the mismatch curve is negative, the PV has a surplus output after supplying the demand. The VPP sells power to the grid and charges up the BESS at the same time. VPP also makes a profit by providing power to 
the grid at the 8 th hour and the 23rd hour when the electricity tariff is high and state of charge is in good condition.

\subsubsection{System Frequency Control}

For load frequency control tests, the execution time step is chosen as one minute, involving load and PV disturbances. Two hours of interest are chosen (9:00 to 10:00 and 15:00 to 16:00). According to the dispatch strategy shown in Fig. 4.6, the battery system is discharging in the first scenario and charging in the second one.

Between 9:00 and 10:00, the real solar output and load curve, as well as their average values are shown in Fig. 4.7(a) and 4.7(b). Fig. 4.7(c) depicts the set point deviations for the CHP and BESS system. As mentioned in the controller design section, the BESS takes over the fast-varying components while the set point deviation for the CHP stays neutral for most of the time. Fig. 4.7(d) compares the system frequency with and without BESSs participation. The light green shaded area indicates permitted safe operation range, which is $\pm 0.2 \mathrm{~Hz}$. After BESSs participation, the system frequency is greatly improved and fluctuates within the safety range for the whole hour.

The second case studies the system response between 15:00 and 16:00, when the generated solar power exceeds the network load demand. Fig. 4.8(a) and Fig. 4.8(b) records the PV output and load curve, Fig. 4.8(c) shows the set point deviations, and the system frequency deviations are shown in Fig. 4.8(d). In Fig. 4.8(a), the actual PV output is much larger than the average value, causing great fluctuations during operation. In Fig. 4.8(d), the CHP fails to regulate system frequency under large disturbances with such a high $\mathrm{PV}$ penetration rate.

\subsubsection{Dynamic Frequency Regulation}

In this section, the execution time step of the MPC-based controller is adjusted to one second. Real-time simulation is conducted to test the effectiveness of the controller under more frequent disturbances. The primary level simulation results are shown in Fig. 4.9 and Fig. 4.10. Similar to the studies with one-minute execution time, the 


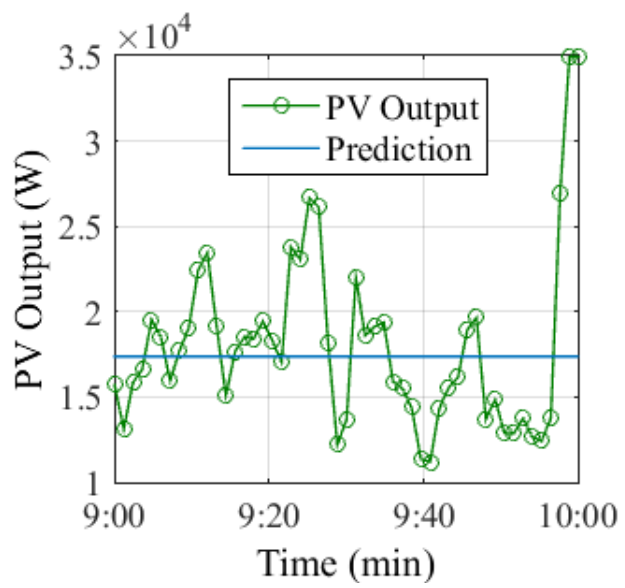

(a) PV output and prediction value.

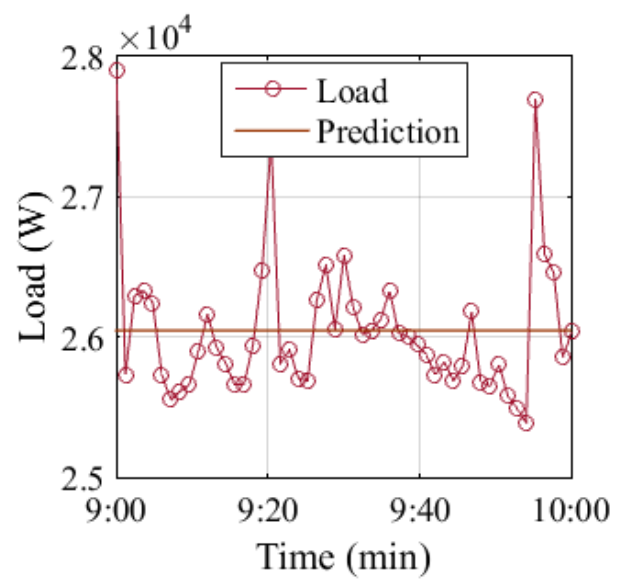

(b) Load power and prediction value.

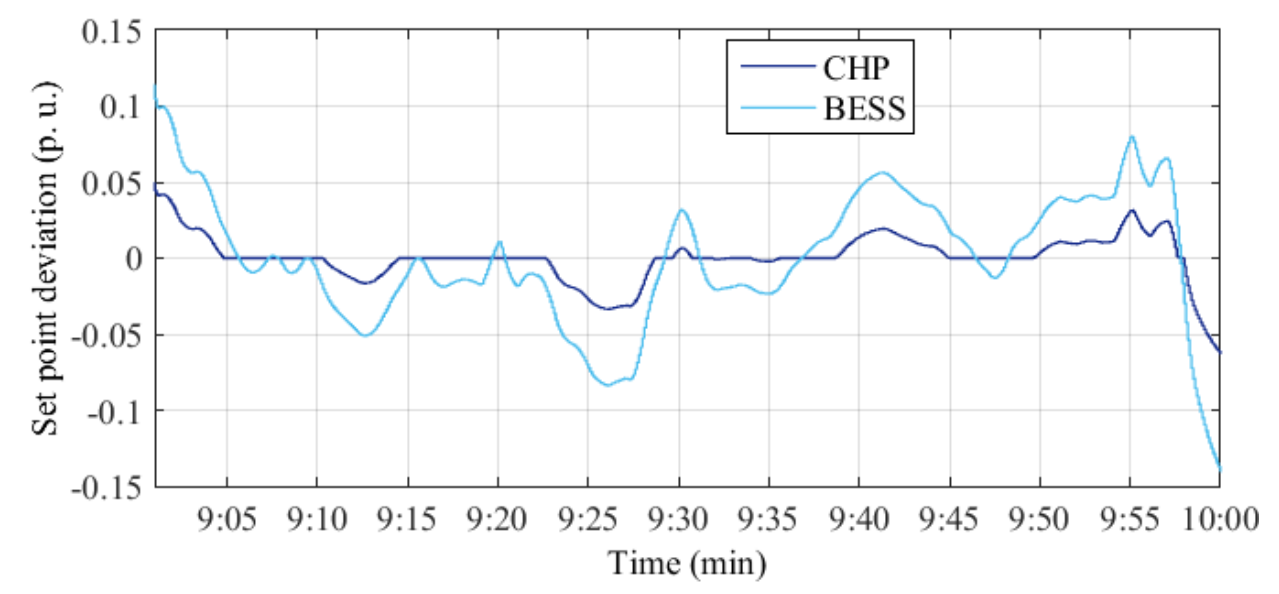

(c) Set point deviations for the CHP and BESS.

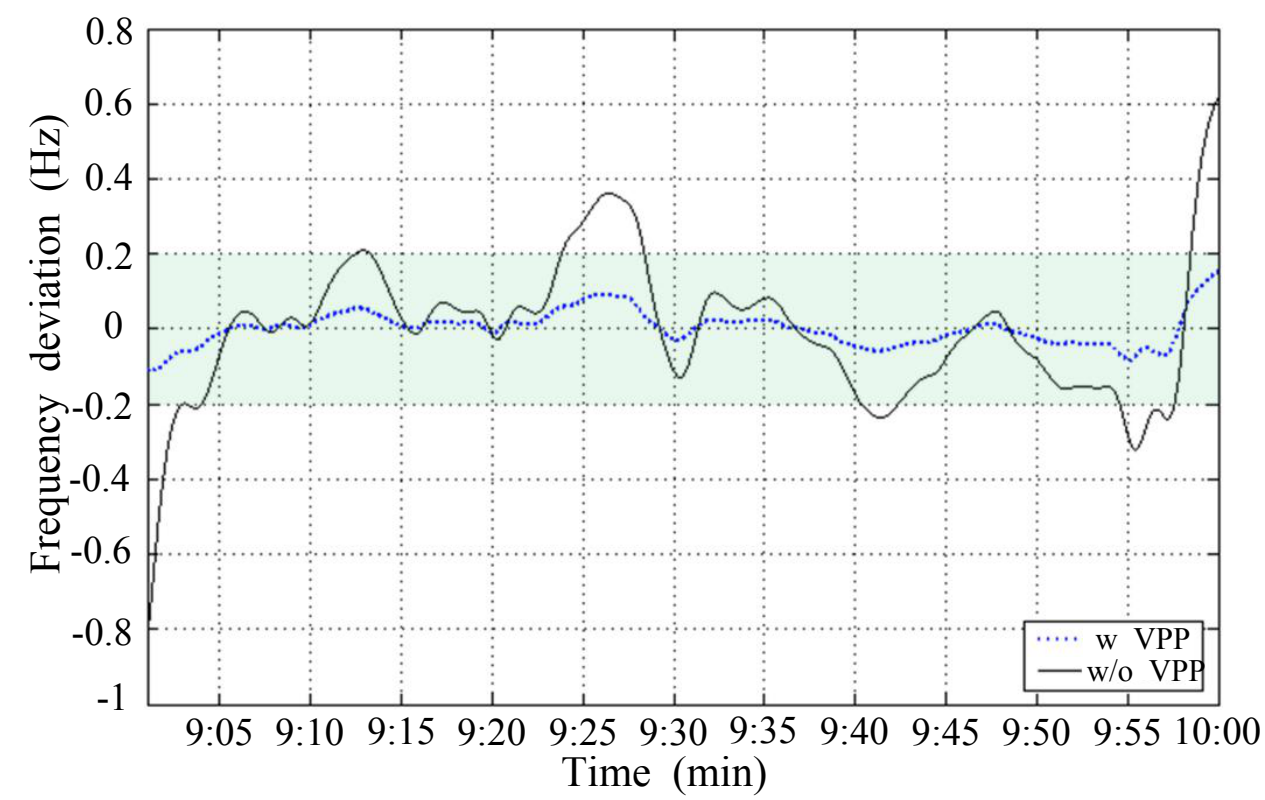

(d) Frequency deviation curve with and without VPP.

Figure 4.7: Simulation results from 9:00 am to 10:00 am. 


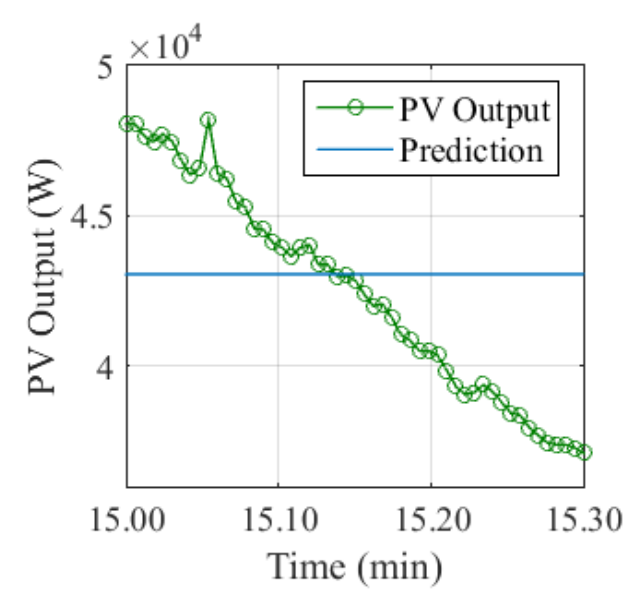

(a) PV output and prediction value.

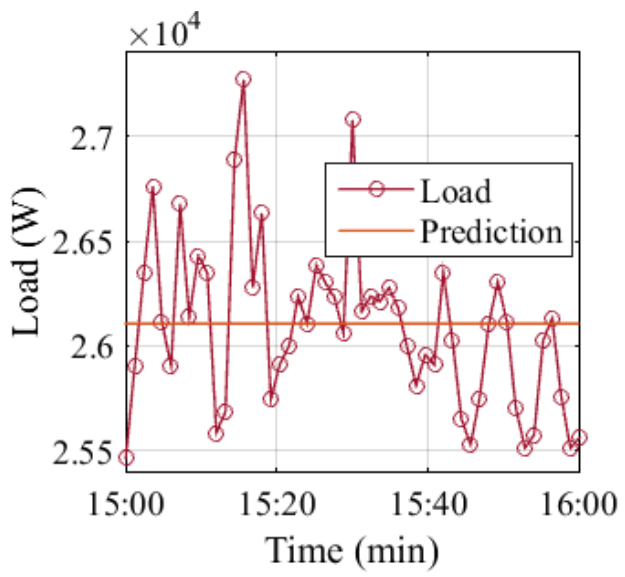

(b) Load power and prediction value.

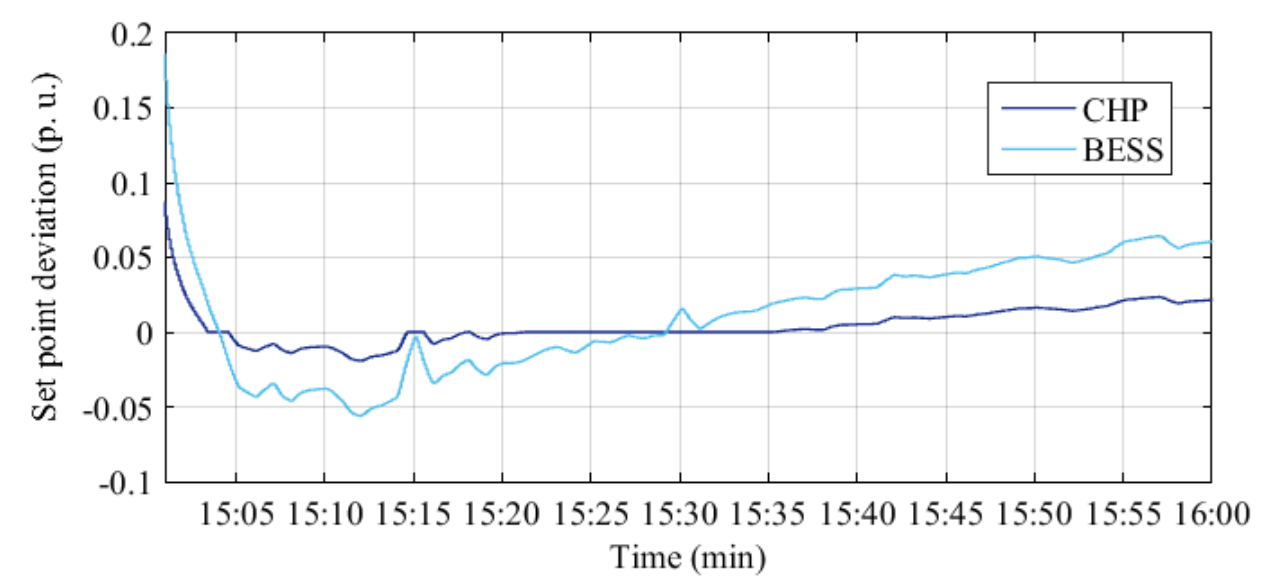

(c) Set point deviations for the CHP and BESS.

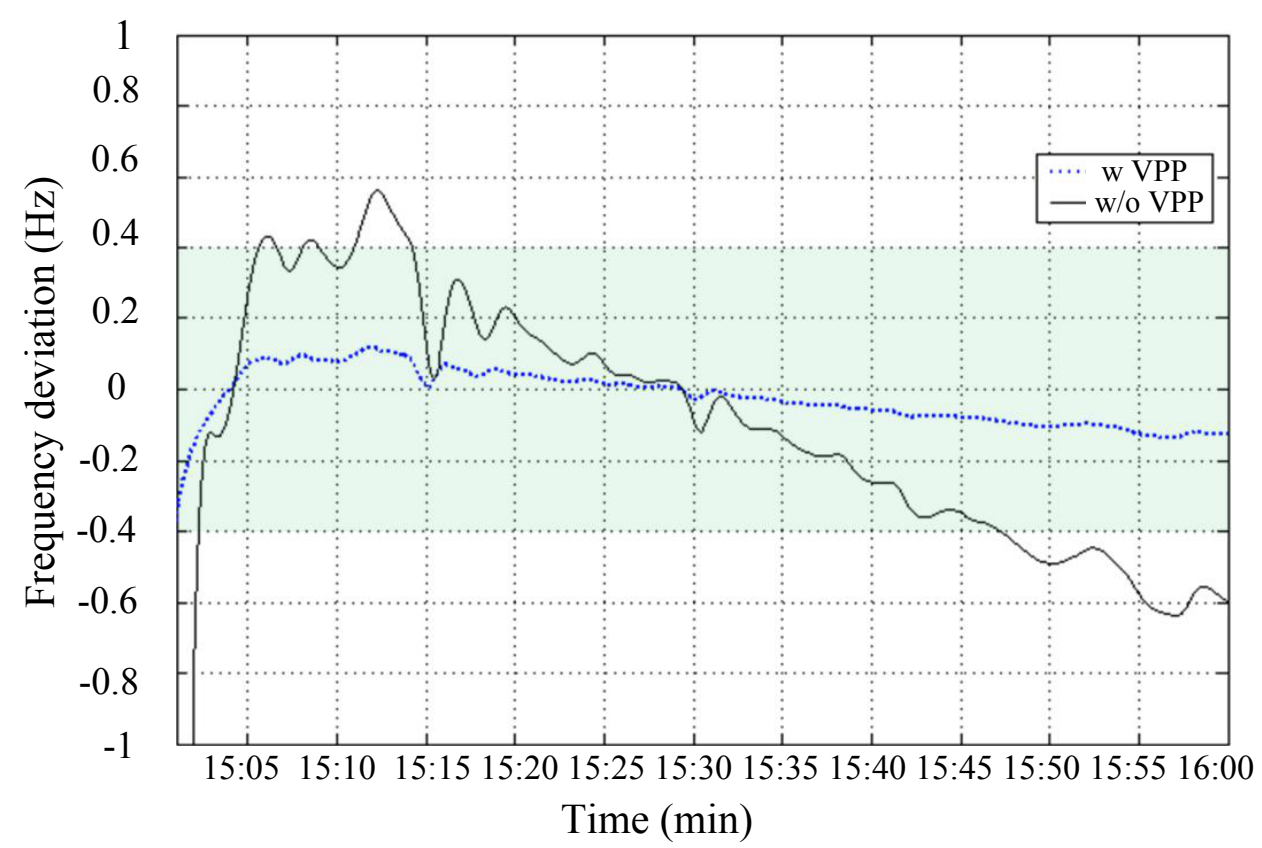

(d) Frequency deviation curve with and without VPP.

Figure 4.8: Simulation results from 15:00 to 16:00. 


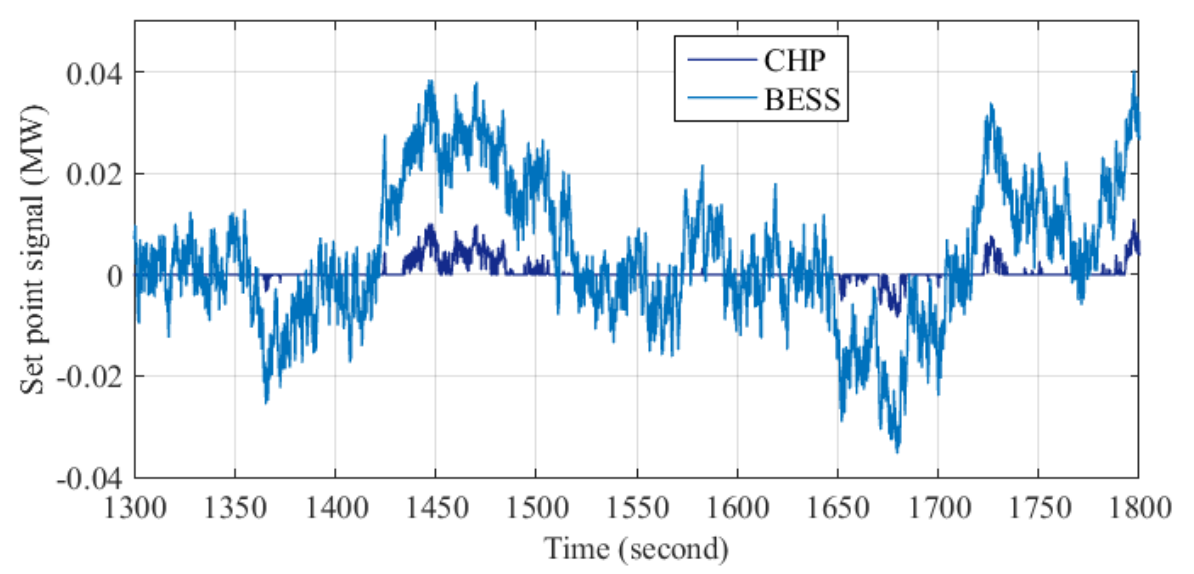

Figure 4.9: Set point deviations for the Generator and BESS from 9:00 to 9:30.

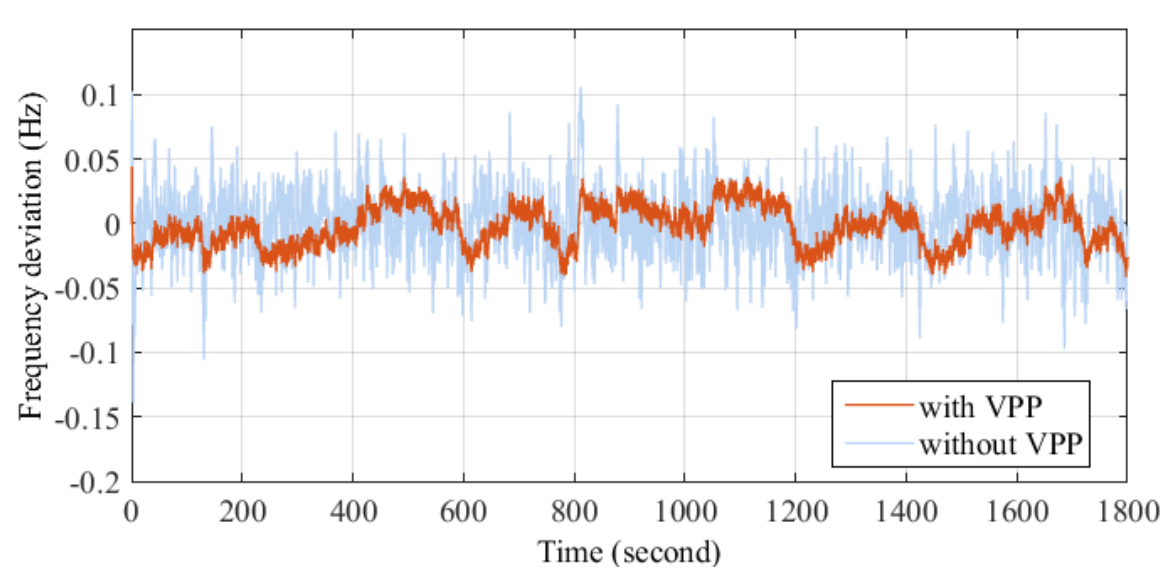

Figure 4.10: Frequency deviation curve with and without VPP .

controller is able to dispatch the power imbalance in every second. In order to get a clearer view of the set point signal, we plot only the last 500 seconds in Fig. 4.9.

The frequency performance with and without VPP is recorded in Fig. 4.10. Obviously, the frequency deviation range with VPP is smaller than the profile without VPP. However, quantitative analysis is still required to measure the improvement.

In order to evaluate and compare the controller effectiveness, we grade the controller performance by the standard deviation value of the system frequency, which is denoted as $\sigma_{f}$. According to the regulations of North American Electricity Reliability Corporation (NERC), $\sigma_{f}$ should be limited within $30 \mathrm{mHz} . \sigma_{f}$ in a $\mathrm{N}$-second time interval is calculated as in (4.10). 
Table 4.1: Standard deviation values without and with VPP

\begin{tabular}{ccc}
\hline & Real-time operation w/o VPP & Real-time operation w VPP \\
\hline Standard deviation & $26.8 \mathrm{mHz}$ & $15.9 \mathrm{mHz}$ \\
\hline$\sigma_{f}=\sqrt{\frac{1}{N^{2}} \sum_{i=1}^{N}\left(\Delta f_{i}\right)^{2}}$
\end{tabular}

For the simulated 30-minute interval, the $\sigma_{f}$ value is calculated and recorded in Table 4.1. The system frequency performance is greatly improved by using the proposed VPP-EMS.

\subsection{Summary}

In this chapter, a hierarchical hybrid MPC controller is proposed for centralized VPP coordination. The proposed controller is composed of two levels, with the primary optimal control being a quadratic regulating controller and the secondary control being a linear economic-oriented dispatch.

A simple power system model is presented, with a CHP power plant, a lumped PV power system and a lumped BESS. Two case studies are implemented with the proposed controller and the modeled power system. The results show that the secondary control executes economic dispatch to coordinate the power dispatch within the VPP according to PV power output, load data, and real-time electricity tariff. In the meantime, the primary controller is capable of stabilizing system frequency within the permitted range during heavy load and peak solar generation periods. 


\section{Chapter 5}

\section{Hierarchical EMS for BESSs in Multiple Markets}

Chapter 4 introduced a centralized frequency controller for a power system with gridconnected BESSs. In this chapter, in order to improve the economic benefit of BESS, several distributed BESSs are aggregated to take part in the electricity market, especially energy market and regulation market. A hierarchical EMS is developed for the optimal scheduling and control of the BESS aggregation.

\subsection{Introduction}

BESSs are gaining increasing research interests in power system applications, for their fast-response ability and control flexibility. Especially when the penetration rate of renewable energy sources increases, more distributed BESSs are installed in various locations in the network to enhance the power quality of the system, especially in low voltage distribution systems $[113,10]$.

Nonetheless, some recent BESS studies state that it is difficult for BESSs to be profitable due to high battery costs [9], even with the proposed optimal sizing scheme [10]. In order to promote the economic value of BESSs, BESSs are maneuvered to participate in market operations. During real-time operations of distributed BESSs, there are mainly two issues. They are the coordination between multiple market operations and the dispatching among distributed BESSs. 
For the first issue, reference [14] optimizes the BESS bidding in the day-ahead energy and spinning reserve market, and reference [15] further includes the ramp-up and rampdown frequency regulation service in the revenue. However, in these optimizers, the assumed high performance is not guaranteed during real-time operations, due to the limited energy capacity of the BESSs and the fluctuating commands. When the system operator sends real-time control signals every few seconds, the actual energy required remains uncertain. In such cases, the participant may fail to get the expected reward.

For such systems, a hierarchical structure is advantageous as claimed by previous research studies $[114,115,116]$. The operational objectives are separated into subcontrollers by their execution periods. Each sub-controller may have its own control algorithm, and exchanges the required information with each other. Reference [114] proposes an MPC-based hierarchical EMS for microgrids incorporating Load Frequency Control, Economic Dispatch, and Unit Commitment within the microgrid. References $[115,116]$ develop double-layered controllers for grid-tied systems with an economic dispatch level to maximize the income and a lower rule-based controller for power balancing. However, these upper level controllers are not designed to include the lower level performance to fulfill the market participation commitment, while both constitute significant weighting factors in the revenue accounting.

For the second issue, due to the minimum capacity requirement from the market regulator, several distributed BESSs need to be aggregated to reach the minimum threshold to enter the market. When multiple BESSs are connected in the same network, coordination between the units helps to fully exploit the storage capacity, and optimize the operation. In the current literature, the coordination of distributed BESSs is not sufficiently addressed. In [17], a conventional "master-slave" logic is used to coordinate different BESSs in the aggregation. This method will result in premature aging of the "master" battery, and will introduce an extra capital investment during the project. The boost of capital return cannot be attained. A second method is proposed by [18], where the control signal distributed to each BESS is proportional to the ratio of its rated power to the overall power of multiple BESSs. This method does not consider the respective SOC, hence resulting in non-response time from some of the BESSs.

To deal with the two issues above, this thesis proposes an MPC-based hierarchical energy management system (HiEMS) for a network with multi-use distributed BESSs, 
taking part in both energy and regulation markets. To generate the best energy arbitrage schedule, a performance constraint is added to the schedule optimization to ensure a high performance score despite the stochastic nature of the regulation signal such that the BESSs can receive high payments.

The proposed HiEMS also attempts to coordinate BESSs of different battery types, various SOC, and power and energy capacity values. The SOC values will be regulated around the expected average SOC to prevent individual saturation or depletion. Price penalties derived from the dynamic annualized cost (DAC) are utilized to dispatch the BESSs, thus increasing the average battery lifetime.

The cost-effectiveness of BESSs using the proposed HiEMS is studied. The costperformance index (CPI) is analyzed for different levels of regulation participation and RES penetration. The CPI values provide a reference for BESS entities to decide the optimal sizing and market participation rate to maximize the income of the multi-use BESSs.

The rest of this chapter is organized as follows: Section 5.2 introduces the HiEMS work flow and major functionalities. Section 5.3 presents the algorithm details in the optimizers. Section 5.4 introduces the simulation environment and set-up in details. Parametric studies for the MPC coefficients are conducted in Section 5.5. Section 5.6 presents case studies based on a local distribution network, and CPI is analyzed. Section 5.5 the control performance of the HiEMS is compared with that of two other decentralized methods. Section 5.6 presents the simulation results for a dynamic strategy, including details for the scenario generation as well. Section 5.7 summarizes the highlights of this chapter.

\subsection{Proposed HiEMS Configuration}

The HiEMS intends to incorporate energy market and regulation market participation. Although market clearing processes generate prices at the same time, the regulation market is sending real-time commands every few seconds. Therefore, the HiEMS works at three levels to comply with different control time schemes: 30 minutes for the energy market schedule, 1 minute for the real-time dispatch, and 2 seconds for the dynamic reg- 


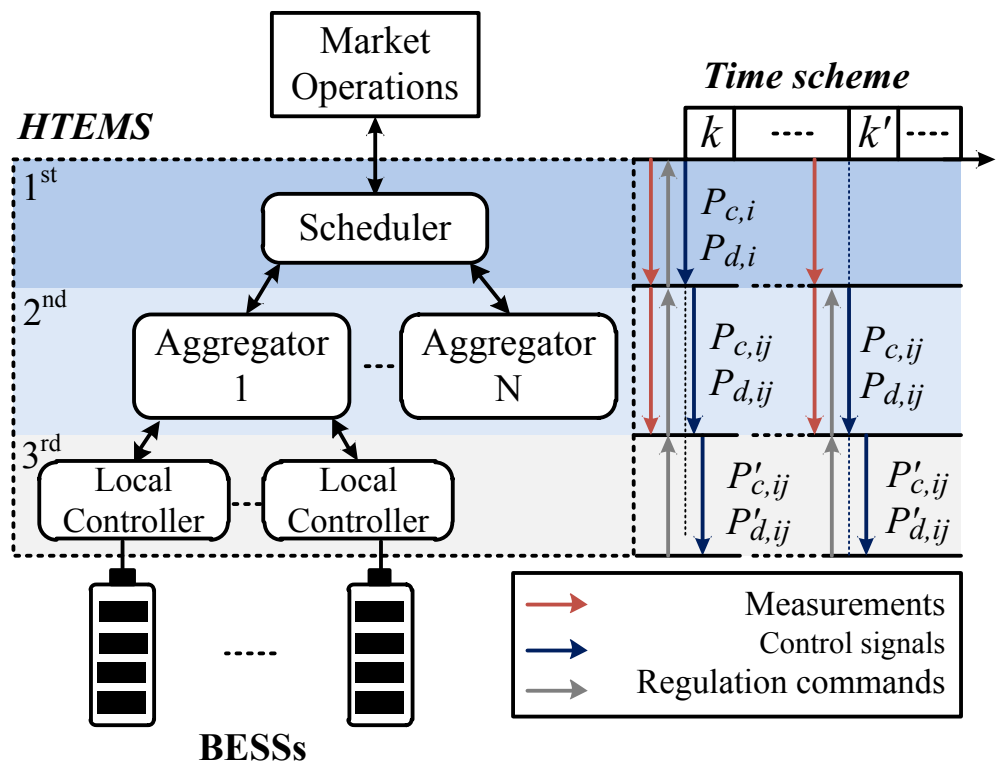

Figure 5.1: Proposed HiEMS integration and its working time scheme.

ulation. The proposed HiEMS integration and its working time scheme are illustrated in Fig. 5.1.

In the HiEMS, several BESSs in a nearby region are aggregated to participate in the markets as one participant. The coordination is conducted by the HiEMS, and thus no communication among the individual BESSs is required. Therefore, the participating resources remain anonymous in the HiEMS. As a result, the privacy of each device can be protected.

Regarding communication issues, the resources need to have appropriate telecommunication and control devices. These are necessities for energy and regulation market participation, regardless of the type of EMS utilized. The first level generates energy set points $P_{i}^{e, c}$ and $P_{i}^{e, d}$ for the aggregations following the market timing scheme; and the second level distributes individual set points $P_{i j}^{c}$ and $P_{i j}^{d}$ optimally according to realtime regulation commands. The work flow and the major functionalities of the proposed HiEMS illustrated in Fig. 5.2. 


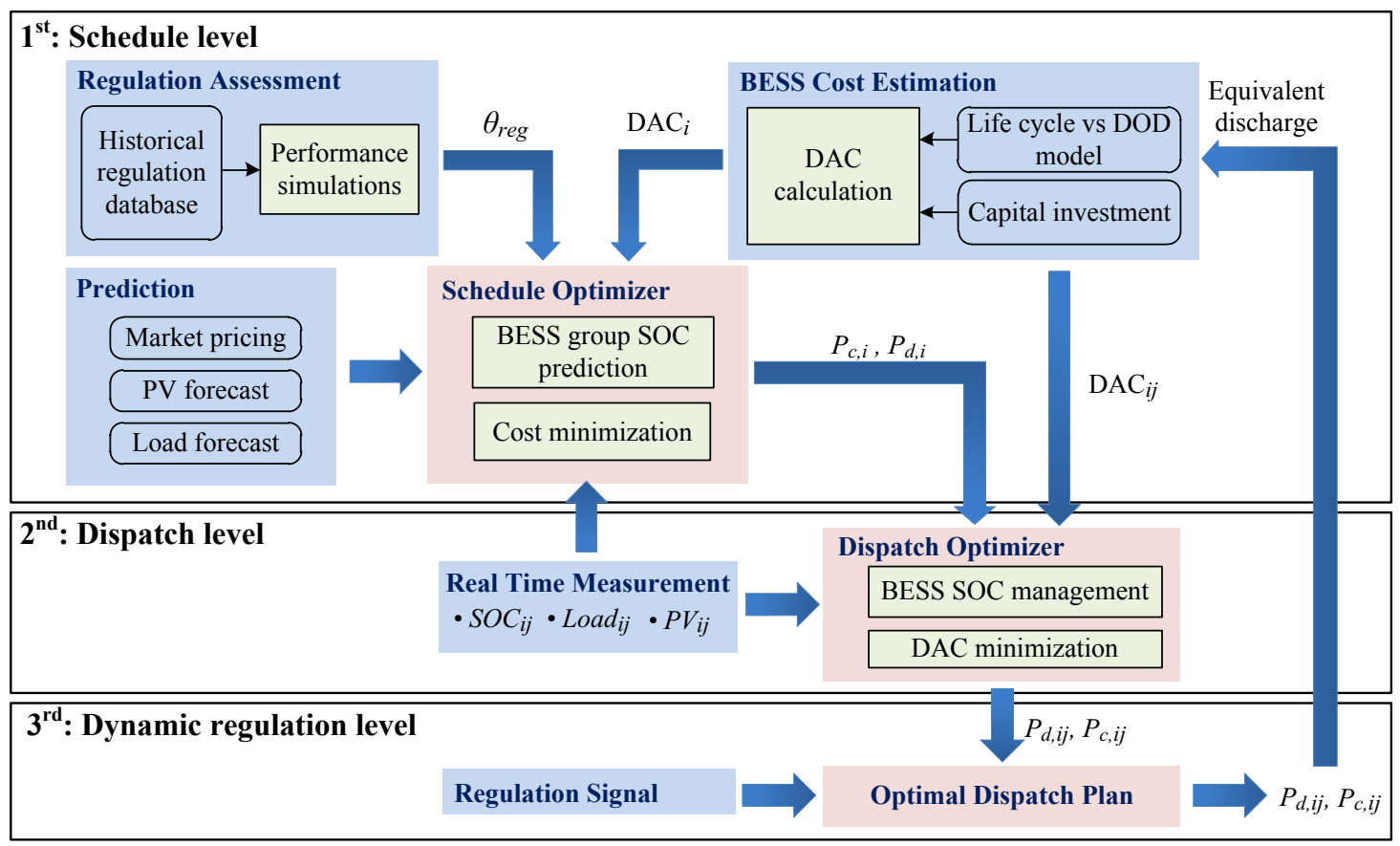

Figure 5.2: Major function modules and data exchanges in the proposed HiEMS.

\subsubsection{Schedule Level}

The schedule level (first level) consists of four modules: namely Regulation Assessment, BESS Cost Estimation, Prediction, and Schedule Optimizer. The key module is the Schedule Optimizer, which is responsible for assessing the cost and revenue of the whole entity, and scheduling the network to achieve a greater economic value during the prediction horizon $H_{p}$ from time $t$ to $t+H_{p}$. The other three modules provide essential information for the decision making in the optimizer.

\subsubsection{Prediction Module}

The Prediction module provides predicted renewable generation, load forecast, as well as market pricing information.

Therefore, the USEP values are predicted using the ANN method. The ANN predictors include price signals and system load consumption of the past seven days, time of the year, time of the week, and time of the day. One year data are used in the ANN training. 


\subsubsection{Regulation Assessment Module}

Frequency regulation units are not forced to perform strictly and rigidly, but a certain level of compliance is required. When the HiEMS commits $\lambda_{r e g}$ of its power to the regulation market, $\theta_{\text {reg }}$ of the BESSs energy should be reserved for regulation to achieve the expected performance.

Unlike renewable generation and load, the regulation signal is highly unpredictable. Simulation tests based on historical data need to be conducted to estimate the amount of energy needed to secure a high performance over the long term. The performance is evaluated using the PJM criteria [45].

\subsubsection{BESS Cost Estimation Module}

The Schedule Optimizer also considers the cost of BESS usage, which is derived from the dynamic annualized cost (DAC). The BESS DAC represents the annualized total BESS capital investment in relation to the on-line estimated battery lifetime $(L T)$. In this chapter, the battery lifetime is estimated given the accumulated usage cycles and predefined depth-of-discharge (DOD).

The BESS Cost Estimation module stores the battery life cycle model, with life cycle data and varying DOD. After reading the DOD measurement, the expected life cycle can be determined from the model. At the same time, the usage cycles during time $t$ can be updated using the battery output $P_{i j, t}^{c}$ and $P_{i j, t}^{d}$ measured with a time step of $\Delta t$, based on the rated energy capacity $E_{B E S S, i}$ as in (5.1). The average battery lifetime (years) for each group $\left(L T_{i}\right)$ can be estimated in (5.2) using total usage cycles.

$$
\begin{gathered}
\text { Usage } \text { cycle }_{i, t}=\frac{\Delta t}{2 \cdot E_{B E S S, i}}\left(\sum_{j} P_{i j, t}^{c}+\sum_{j} P_{i j, t}^{d}\right) \\
L T_{i}=\text { Life } \operatorname{cycle}_{i} / \sum_{t} \text { Usage cycle }_{i, t}
\end{gathered}
$$

The BESS capital cost data are also stored in this module. For BESSs with rated power $P_{B E S S, i}$ and rated energy $E_{B E S S, i}$, the cost consists of power investment and 
energy investment as in (5.3). Group $D A C_{i}$ equals the total cost multiplying the capital recovery factor $C R F_{i}$ in (5.4) and (5.5), where the interest rate $r$ is set as 0.06 .

$$
\begin{gathered}
\text { Total cost }{ }_{i}=c_{k W, i} \cdot P_{B E S S, i}+c_{k W h, i} \cdot E_{B E S S, i} \\
C R F_{i}=\frac{r \cdot(1+r)^{L T_{i}}}{(1+r)^{L T_{i}}-1} \\
D A C_{i}={\text { Total } \text { cost }_{i} \cdot C R F_{i}}
\end{gathered}
$$

\subsubsection{Dispatch and Dynamic Regulation Levels}

The dispatch level (second level) and the dynamic regulation level (third level) work together to deal with the real-time fluctuation in the network, and respond to the regulation commands from the aggregator.

\subsubsection{Dispatch Level}

The dispatch level (second level) deals with group set point distribution during the prediction horizon from time $t^{\prime}$ to $t+H_{p}^{\prime}$. After receiving $P_{i}^{d}$ and $P_{i}^{c}$ from the first level, the Dispatch Optimizer in the second level aims to distribute $P_{i j}^{d}$ and $P_{i j}^{c}$ to each BESS in the group.

When dispatching the power set point, the objective of Dispatch Optimizer includes two parts: minimizing the BESS cost using the DAC from the first level and managing the SOC values to avoid saturation or depletion of any BESS.

\subsubsection{Dynamic Regulation}

In regulation market operations, BESSs can respond to the slower-varying traditional regulation signal or the dynamic regulation signal. The regulation signals are obtained from the ancillary service database on the PJM official website. 
In the HiEMS, the regulation commands are distributed to each BESS in proportion to the Optimal Dispatch Plan $P_{d, i j}$ and $P_{c, i j}$ updated every minute by Dispatch Optimizer in the second level. The dynamically adjusted control signals $P_{d, i j}^{\prime}$ and $P_{c, i j}^{\prime}$ are sent to the local controllers on the devices.

\subsection{Optimizer Formulations}

In this section, the optimizers in the first and second levels are described in detail. As necessities for energy and regulation market participation, appropriate telecommunication and control devices will be installed, and provide access to updated measurements. Therefore, the optimizers can be implemented using the predictive control scheme to provide better real-time performance.

\subsubsection{First Level Schedule Optimizer}

\subsubsection{State-space Modeling}

In the Schedule Optimizer of first level, control inputs are defined as the charging and discharging commands for each BESS group:

$$
\mathbf{u}(k)=\left[P_{d, 1}(k), P_{c, 1}(k), \ldots, P_{d, n}(k), P_{c, n}(k)\right]^{T}
$$

To apply distinctive parameters during charging and discharging in the model, the two processes are labeled as positive variables $P_{c, i}$ and $P_{d, i}$ for the $i^{\text {th }}$ group, and are constrained by $P_{c, i} \cdot P_{d, i}=0$ to avoid illogical schedules.

The state variables are chosen as the average SOC of the groups:

$$
\mathbf{x}(k)=\left[S O C_{1}, S O C_{2}, \ldots S O C_{n}\right]^{T}
$$

The overall HiEMS electricity exchange $P_{\text {grid }}$ with the main grid is chosen as the system output:

$$
\mathbf{y}(k)=P_{\text {grid }}
$$


Since the load and PV are assumed to be uncontrollable, the predicted load consumption $P_{\text {load }}$ and PV production $P_{P V}$ are fed into the model as system disturbances as in (5.9).

$$
\boldsymbol{\omega}(k)=\left[P_{\text {load }}(k), P_{P V}(k)\right]^{T}
$$

The average SOC of each group is predicted by coulomb-counting method [103], considering different charging and discharging efficiencies for various battery types shown in $(5.10)$.

$$
S O C_{i}(k+1 \mid k)= \begin{cases}S O C_{i}(k)-\frac{P_{d, i} \cdot \Delta t}{\eta_{d, i} \cdot E_{B E S S, i}} & \text { discharging } \\ S O C_{i}(k)+\frac{\eta_{c, i} \cdot P_{c, i} \cdot \Delta t}{E_{B E S S, i}} & \text { charging }\end{cases}
$$

The electricity exchange $P_{\text {grid }}$ is predicted using the power balance constraint in the network at each time step as in (5.11). The HiEMS controls nearby BESS units in a distribution network, where grid transmission losses are negligibly small, and grid transmission constraints are non-binding.

$$
\begin{aligned}
P_{\text {grid }}(k+t \mid k) & =P_{\text {load }}(k+t \mid k)-P_{P V}(k+t \mid k) \\
& +\left\{\sum_{i} P_{c, i}(k+t \mid k)-\sum_{i} P_{d, i}(k+t \mid k)\right\}
\end{aligned}
$$

Therefore, the state-space matrices are formulated as follows:

$$
\begin{gathered}
A_{n \times n}=\mathbf{I}^{n \times n}, \\
B_{n \times 2 n}=\left[\begin{array}{ccccc}
\frac{-\Delta t}{\eta_{d, 1} \cdot E_{B E S S, 1}} & \frac{\eta_{c, 1} \cdot \Delta t}{E_{B E S S, 1}} & \cdots & 0 & 0 \\
0 & 0 & \cdots & 0 & 0 \\
\vdots & \vdots & \ddots & \vdots & \vdots \\
0 & 0 & \cdots & \frac{-\Delta t}{\eta_{d, n} \cdot E_{B E S S, n}} & \frac{\eta_{c, n} \cdot \Delta t}{E_{B E S S, n}}
\end{array}\right], \\
D_{1 \times 2 n}=\left[\begin{array}{lllll}
-1 & 1 & \ldots & -1 & 1
\end{array}\right], W_{1 \times 2}=\left[\begin{array}{ll}
1 & -1
\end{array}\right]
\end{gathered}
$$


When the buying and selling prices are different, the amount of buying and selling of electricity $\left(z_{\text {sell }}\right.$ and $\left.z_{\text {buy }}\right)$ are generated from the system output $P_{\text {grid }}$ using a nonlinear function:

$$
\begin{aligned}
\mathbf{z}(k) & =\left[z_{\text {sell }}(k), z_{\text {buy }}(k)\right]^{T} \\
& =\mathcal{F}(y)= \begin{cases}{\left[\begin{array}{ll}
0 & y
\end{array}\right]^{T}} & \text { when } y \geq 0, \text { buying; } \\
{\left[\begin{array}{ll}
-y & 0
\end{array}\right]^{T}} & \text { when } y \leq 0, \text { selling. }\end{cases}
\end{aligned}
$$

\subsubsection{Optimization Formulation}

The scheduling objective function $V_{1}$ aims to schedule the BESSs aggregators to maximize the overall network profit, which consists of the electricity bill savings and the BESS usage costs.

The objective function is defined as follows:

$$
V_{1}:=\min _{\mathbf{u}} \sum_{t=0}^{H_{p}} \underbrace{\left[\mathbf{c}_{\text {grid }}(k+t \mid k) \cdot \mathbf{z}(k+t \mid k)\right]}_{\text {Electricity bill }}+\sum_{t=0}^{H_{u}} \underbrace{\left[\mathbf{c}_{\text {bat }}(k) \cdot \mathbf{u}(k+t \mid k)\right]}_{\text {Battery cost }}
$$

subject to

$$
\begin{aligned}
& \mathbf{x}(k+t \mid k)=A \mathbf{x}(k+t-1 \mid k)+B \mathbf{u}(k+t \mid k) \\
& \mathbf{u}(k+t \mid k) \in \mathbf{P}_{\mathrm{BESS}} \quad \forall t \in\left[1, H_{u}\right] \\
& S O C_{l b, i}+\theta_{\text {reg }, i} \leq S O C_{i}(k+t) \leq S O C_{u b, i}-\theta_{\text {reg }, i} \\
& S O C_{i}\left(k+H_{p} \mid k\right)=0.5 \quad \forall i \in[1, n]
\end{aligned}
$$

where $\mathbf{c}_{\text {grid }}$ is a vector storing the future electricity prices; $\mathbf{c}_{\mathbf{b a t}}$ is a vector with BESS DAC data; $\mathbf{P}_{\mathbf{B E S S}}$ is a vector with all BESS power ratings; and $S O C_{u b, i}$ and $S O C_{l b, i}$ represent the respective upper bound and lower bound of the group SOC. 


\subsubsection{Second level Dispatch Optimizer}

\subsubsection{State-space Modeling}

The state-space model of the second level is similar to that of the second level optimizer except that the dynamic of every individual BESS will be taken into consideration, and the dimensions of the system matrices will be expanded accordingly.

The control inputs are taken as:

$$
\mathbf{u}^{\prime}\left(k^{\prime}\right)=\left[\mathbf{u}_{\mathbf{1}}^{\prime}, \ldots, \mathbf{u}_{\mathbf{n}}^{\prime}\right]^{T}
$$

where $\mathbf{u}_{\mathbf{i}}^{\prime}\left(k^{\prime}\right)=\left[P_{d, i 1}, P_{c, i 1}, \ldots, P_{d, i m_{i}}, P_{c, i m_{i}}\right]$.

The $S O C_{i j}$ values are chosen as the state variables in (5.15), where $\mathbf{S O C}_{\mathbf{i}}$ represents all the individual SOC values in the $i^{t h}$ group: $\mathbf{S O C}_{\mathbf{i}}=\left[S O C_{i 1}, \ldots, S O C_{i m_{i}}\right]$.

$$
\mathbf{x}^{\prime}\left(k^{\prime}\right)=\left[\mathbf{S O C}_{\mathbf{1}}, \ldots, \mathbf{S O C}_{\mathbf{n}}\right]^{T},
$$

The output variable is the fluctuation of the real system output $\tilde{P}_{\text {grid }}$ from the scheduled system output $P_{\text {grid }}$ :

$$
\mathbf{y}^{\prime}\left(k^{\prime}\right)=\Delta P_{\text {grid }}=\tilde{P}_{\text {grid }}-P_{\text {grid }}
$$

\subsubsection{Optimization Formulation}

The objective function includes three terms as shown in (5.17). The first term minimizes the battery DAC costs. The second term manages the SOC towards the predicted group average level. The third term adjusts the real-time power output to minimize $\Delta P_{\text {grid }}$, the fluctuation from its scheduled value. The three objectives are combined by weighting factors $\alpha_{1}$ and $\alpha_{2}$. The norm used in the equation is the absolute value norm. 


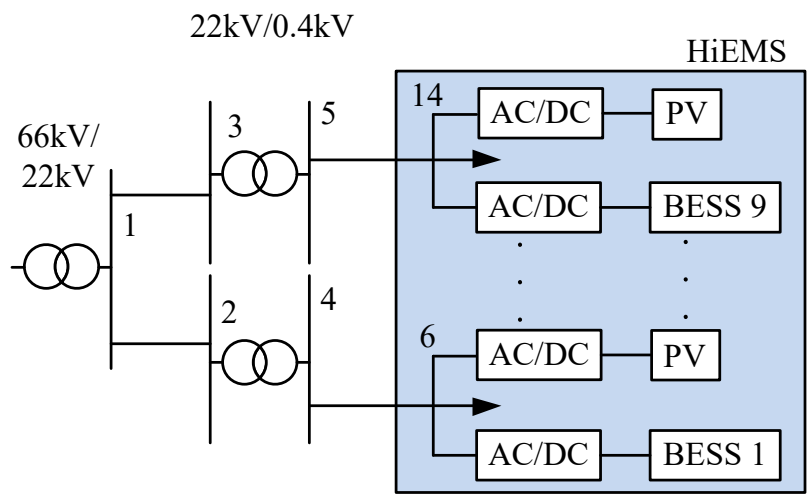

Figure 5.3: A Singaporean 14-bus radial distribution network.

$$
\begin{aligned}
V_{2}:=\min _{\mathbf{u}^{\prime}} \sum_{t=0}^{H_{u}^{\prime}} \sum_{i=1}^{n} \underbrace{\left[\mathbf{c}_{\mathbf{b a t}, \mathbf{i}}\left(k^{\prime}\right) \cdot \mathbf{u}_{\mathbf{i}}^{\prime}\left(k^{\prime}+t \mid k^{\prime}\right)^{T}\right]}_{i^{t h} \text { group battery cost }} & +\alpha_{1} \underbrace{\sum_{t=0}^{H_{p}^{\prime}} \sum_{i=1}^{n} \sum_{j=1}^{m_{i}}\left\|S O C_{i j}-S O C_{i}\right\|^{2}}_{S O C \text { management }} \\
& +\alpha_{2} \underbrace{\sum_{t=0}^{H_{p}^{\prime}}\left\|\Delta P_{\text {grid }}\right\|}_{\text {Real-time fluctuations }}
\end{aligned}
$$

subject to

$$
\begin{aligned}
& \mathbf{u}^{\prime}\left(k^{\prime}+t\right) \in \mathbf{P}_{\mathrm{BESS}} \quad \forall t \in\left[0, H_{p}^{\prime}\right] \\
& S O C_{l b, i} \leq S O C_{i j}\left(k^{\prime}+t\right) \leq S O C_{u b, i}
\end{aligned}
$$

\subsection{Simulation Environment}

The proposed HiEMS is simulated based on a 14-bus Singaporean distribution system shown in Fig. 5.3. Buses from number 6 to number 14 are load buses connected to residential buildings. Each building is equipped with a BESS.

The Uniform Singapore Energy Price (USEP) in Fig. 5.4 is used as the real-time buying and selling price. The USEP applies to prosumers for all energy injections or withdrawals that occur in the Singapore power system. The market clearing engine generates the USEP half-hourly [117]. 


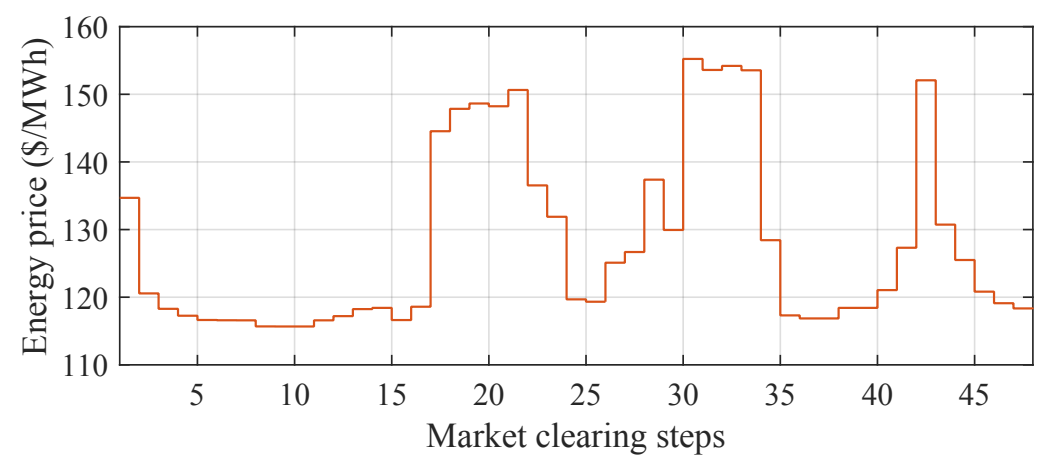

Figure 5.4: Simulation pricing data.

Table 5.1: Settings of BESSs

\begin{tabular}{|c|c|c|c|c|c|c|c|c|c|c|}
\hline \multirow{2}{*}{\multicolumn{2}{|c|}{ BESS index }} & \multicolumn{4}{|c|}{ Vanadium group (V) } & \multicolumn{5}{|c|}{ Lithium group (L) } \\
\hline & & 1 & 4 & 5 & 6 & 2 & 3 & 7 & 8 & 9 \\
\hline \multicolumn{2}{|c|}{ Bus number } & 6 & 9 & 10 & 11 & 7 & 8 & 12 & 13 & 14 \\
\hline \multicolumn{2}{|c|}{ Power (kW) } & 120 & 80 & 100 & 80 & 40 & 20 & 40 & 20 & 20 \\
\hline \multicolumn{2}{|c|}{ Energy $(\mathrm{kWh})$} & 360 & 400 & 600 & 320 & 120 & 100 & 160 & 120 & 200 \\
\hline \multicolumn{2}{|c|}{$\mathrm{SOC}_{0}(\%)$} & 40 & 60 & 80 & 20 & 38 & 48 & 34 & 71 & 32 \\
\hline \multirow{2}{*}{ Efficiency } & $\eta_{d}$ & \multicolumn{4}{|c|}{0.88} & \multicolumn{5}{|c|}{0.9} \\
\hline & $\eta_{c}$ & \multicolumn{4}{|c|}{0.8} & \multicolumn{5}{|c|}{0.85} \\
\hline \multirow{2}{*}{ Investment } & $\$ / \mathrm{kW}$ & \multicolumn{4}{|c|}{600} & \multicolumn{5}{|c|}{1200} \\
\hline & $\$ / \mathrm{kWh}$ & \multicolumn{4}{|c|}{100} & \multicolumn{5}{|c|}{600} \\
\hline
\end{tabular}

The BESSs are grouped by two widely used battery types: Vanadium (V group) and Lithium-ion (L group). Detailed BESS settings are listed in Table 5.1.

The optimizers are modeled in General Algebraic Modeling System (GAMS), and the binary nonlinear optimizations can be solved by KNITRO solver. The iteration limit defined in GAMS is usually 2,000,000,000 iterations, and the optimality tolerance for the reduced gradient is 1e-7. The full set of parameter settings can be found at the GAMS website [118].

The work flow and system simulation of HiEMS are built in Matlab. The optimizers and the simulation platform are interfaced using GDXMRW [119]. The whole simulation scheme is illustrated in Fig. 5.5. The simulation platform is done in Matlab, and the optimization is modeled in GAMS. For each time instance, Matlab simulation feeds GAMS optimization with data needed, and will wait for the optimization problem to be solved. The optimization problem generally takes around 1 second to obtain its solution. 


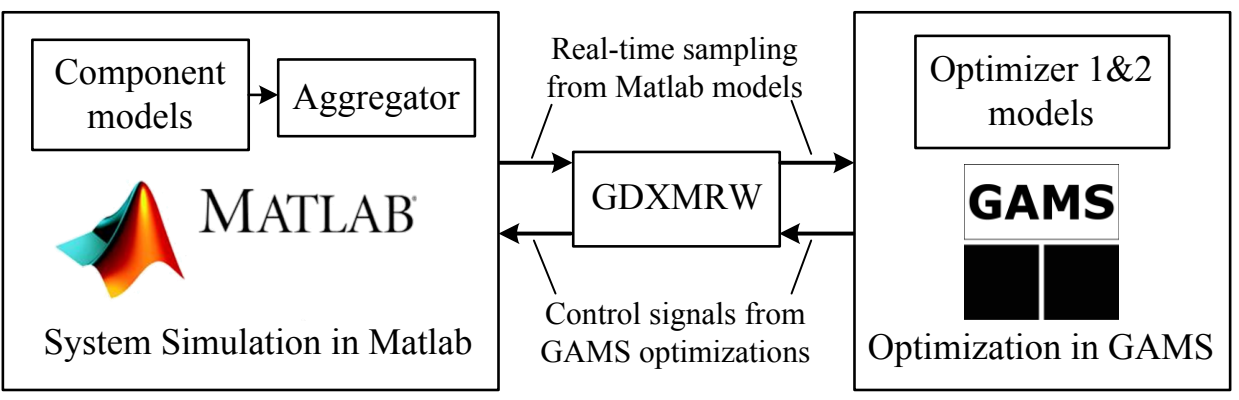

Figure 5.5: Schematic diagram of system simulations.

\subsection{Parametric Studies}

In this section, parametric studies are carried out to optimize the MPC scheme parameters and the optimization coefficients in order to guarantee problem optimality.

\subsubsection{MPC Prediction Horizon Analysis}

In the MPC formulation, each output from solving the optimization repetitively is nontrivial especially with the presence of prediction errors. For example, intuitively speaking, the scheduler should be making more profit when the prediction horizon is longer, since more future information is known ahead of time and better decisions can be made. However, when perfect predictions are not attainable, prediction errors can result in deviations from the optimal schedule, which in return, harm the total profit. Therefore, to reach a comparatively optimal strategy, parametric studies should be carried out when dealing with different prediction technologies in the MPC formulation.

To demonstrate the role of MPC in handling prediction errors, test cases with load predictions are conducted.

Firstly, a load forecast model using ANN is built, to predict the load profile with different lead time values. The predictors used in the model are historical load power of the past seven days, average load power of the previous day, time of the day, day of the week and time of the year. The 2-year load historical data are downloaded from the Singapore EMC website, and scaled down to fit our test cases. The mean absolute percentage error for selected lead times are recorded in the following bar graph as shown in Fig. 5.6. According to Fig. 5.6, MAPE generally increases with longer lead time 


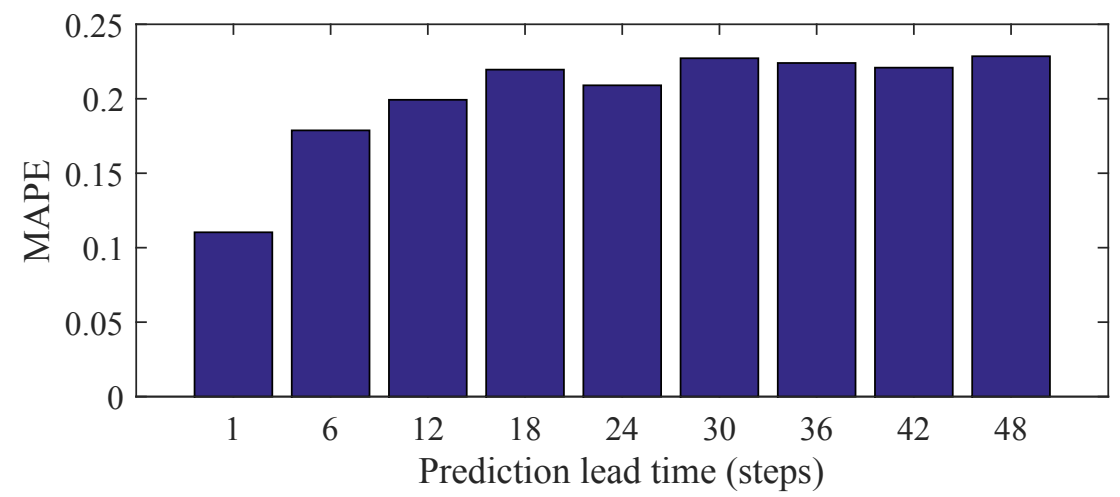

Figure 5.6: MAPE with regards to different prediction lead time values.

Table 5.2: Estimated annual revenue with varying lead time values

\begin{tabular}{|c|c|}
\hline Lead time value (hours) & Estimated annual revenue (\$) \\
\hline 6 & 20,645 \\
\hline 12 & 24,866 \\
\hline 15 & 30,772 \\
\hline 18 & 31,715 \\
\hline 21 & 27,759 \\
\hline 24 & 27,314 \\
\hline
\end{tabular}

values from 0.5 to 9 hours, and stays around similar values after 9 hours.

With the prediction data, parametric studies are performed for different lead time values. In each case, a lead time value is assigned to the load prediction model, and this lead time value is also set as the prediction horizon for the MPC optimizer. In each optimization step, the respective BESS schedule is updated based on the given load prediction.

The resulting income from the respective schedules are estimated and recorded in Table 5.2. When increasing the lead time value from 6 hours to 18 hours, the estimated annual revenue is increasing generally. This indicates that the future information is benefiting the revenue, although certain levels of prediction errors exist. However, after 18 hours, the estimated annual revenue drops. The discrepancy between the expected load profile and the real load consumption starts to show its impact on the overall revenue. Therefore, for the prediction model we developed, 18 hours would be the best lead time for our schedule level optimizer. For other prediction methods, similar parametric analysis can be done to maximize the possible revenue. 


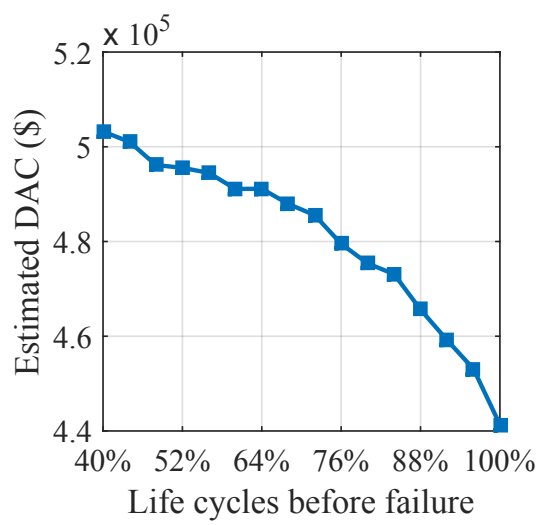

(a) Parametric studies for DAC.

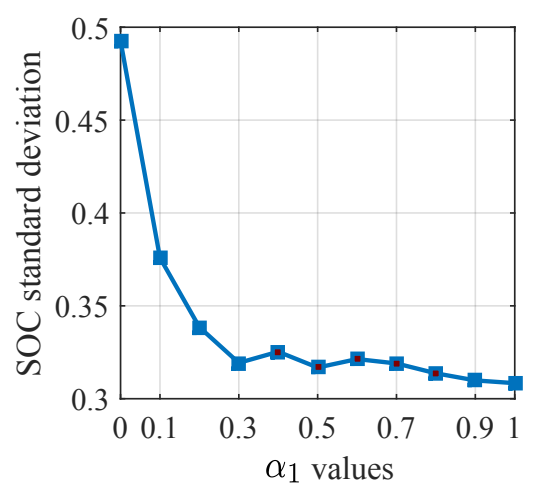

(c) Parametric studies for $\alpha_{1}$.

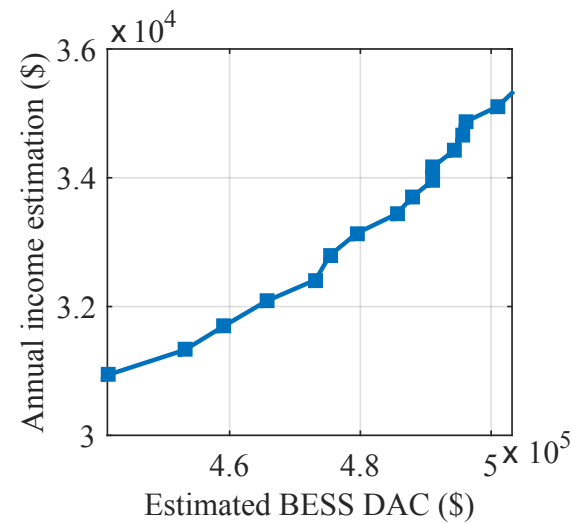

(b) Solution sets for the first level optimizer.

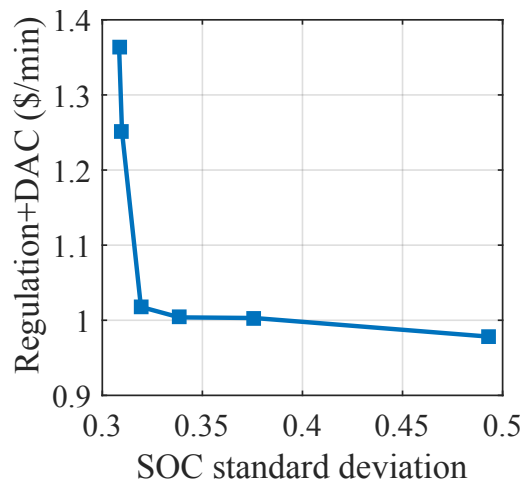

(d) Solution sets for second level optimizer.

Figure 5.7: Parametric studies for Pareto optimality.

\subsubsection{Pareto Optimality Analysis}

The optimization models proposed in (5.13) and (5.17) are multi-objective optimizations scalarized into single-objective ones. In this subsection, the optimality efficiency is demonstrated by parametric studies and posterior optimization tests.

In the first level, the three objectives (electricity bill, regulation revenue, and battery cost), are commeasurable and are converted into a single-objective optimization problem weighted by their prices. The DAC value is dependent on the total cycles setting. As shown in Fig. 5.7(a), the higher the life cycles are allowed before battery failure, the lower the DAC will be. Figure 5.7(b) shows the equilibrium between the DAC and total annual income. To further enhance the economic gain from the markets, the battery cost has to be increased due to a result of a more frequent BESS utilization; and to 
Table 5.3: Optimizer parameters

\begin{tabular}{|c|c|c|c|c|c|}
\hline Level & $\Delta t$ & $H_{p}$ & $H_{u}$ & $\alpha_{1}$ & $\alpha_{2}$ \\
\hline first & $0.5 \mathrm{~h}$ & $18 \mathrm{~h}$ & $18 \mathrm{~h}$ & \multicolumn{2}{|c|}{ N.A. } \\
\hline second & $1 \mathrm{~min}$ & $1 \mathrm{~min}$ & $1 \mathrm{~min}$ & 0.2 & USEP \\
\hline
\end{tabular}

decrease battery cost, the BESS has to reduce market operation. In other words, The HiEMS cannot make more profit from the markets and decrease the DAC at the same time. Therefore, Pareto efficiency can be obtained in the optimization.

However, in the second level optimizer, the SOC management objective is not measurable by economic values. The respective weighting usually complies with a preference set by the HiEMS operator. In the parametric studies recorded in Fig. 5.7(c), the standard deviation of the SOC values can be reduced greatly with $\alpha_{1}$ larger than 0.2 . The $\alpha_{1}$ values are used in the optimality tests, and the equilibrium is shown in Fig. 5.7(d). A reduction of regulation penalty and DAC is traded off for an increase in SOC deviations from the expected value.

\subsection{Simulation Studies}

In the base case study, no PV integration is involved. The HiEMS commits 0.1 of its rated power into regulation, i.e. $\lambda_{\text {reg }}=0.1$. The regulation performance constraint $\theta_{\text {reg }}$ is set as 0.01, resulting from the Regulation Assessment simulations. The optimizer parameters chosen are listed in the following Table 5.3.

\subsubsection{4-hour Operation Results}

The simulated schedule from the first level is plotted in Fig. 5.8. Since the DAC is always lower than USEP, the HiEMS tends to store energy from the grid during low USEP periods, such as the time period from 3:30 to 5:00, and supply the loads using BESSs during high USEP periods, such as from 14:30 to 15:00 and from 15:30 to 16:00.

The simulated real-time system consumption with and without HiEMS are also plotted in Fig. 5.8. The overall HiEMS curve follows the schedule and regulation commands steadily despite load fluctuations throughout the entire period. Only when the fluctuation is much higher than expected, the HiEMS will no longer be able to sustain power 


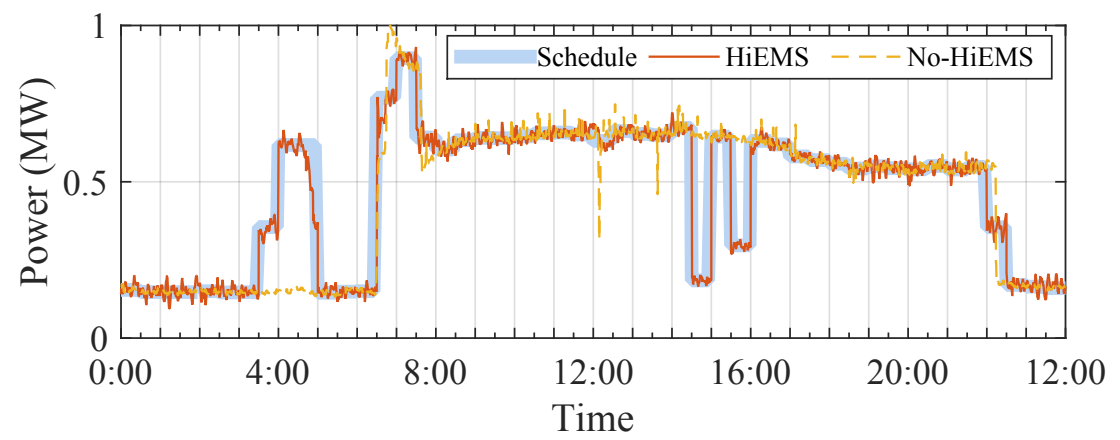

Figure 5.8: System consumption schedule, and real-time curves with and without HiEMS.

arbitrage or handle the disturbances (e.g. approaching 5:00).

The overall amount of low performance time is well within the tolerance. A high overall regulation score of $91.7 \%$ is obtained. BESSs are coordinated to achieve the high performance. First, BESS is dispatched in accordance with DAC. In Fig. 5.9(a) and Fig. 5.9(b), the L group BESSs are utilized less frequently than the V group due to their higher costs. Second, the SOC penalty in the objective function drives every BESS towards the predicted average SOC, regardless of the initial SOC values. This SOC management can be observed in Fig. 5.9.

\subsubsection{CPI Improvement of BESS}

CPI represents the economic benefit returns from the investment. The revenue of the BESS aggregation comprises two parts: energy market income/savings and regulation revenue, shown in (5.18). The energy market income equals the electricity bill saving after engaging the HiEMS. The total annual cost for the distributed BESSs is the sum of the average $\overline{D A C}_{i}$ for each group calculated in (5.5), and the general pricing information from the literature $[11,12,13]$ is summarized in Table 5.1.

$$
\mathrm{CPI}=\frac{\text { Earned Value }}{\text { Actual Cost }}=\frac{\overbrace{R_{C}+R_{P}}^{\text {Regulation income }}+\text { Energy income }}{\sum_{i=1}^{n}{\text { Total } \text { cost }_{i}}}
$$

The regulation income has two components: $R_{C}$ and $R_{P} . R_{C}$ is the capacity revenue for reserving the capacity, and $R_{P}$ is the performance revenue. Detailed formulations 


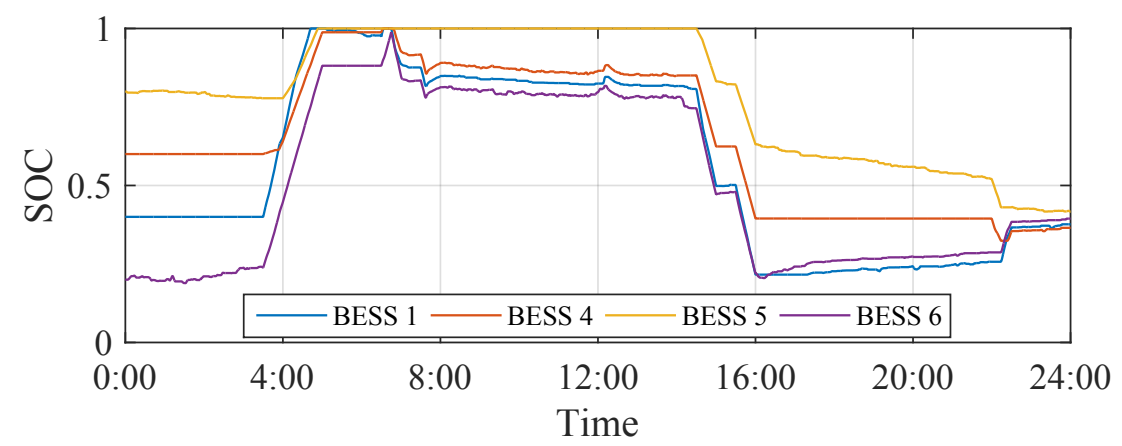

(a) SOC curves of Vanadium BESSs (V group).

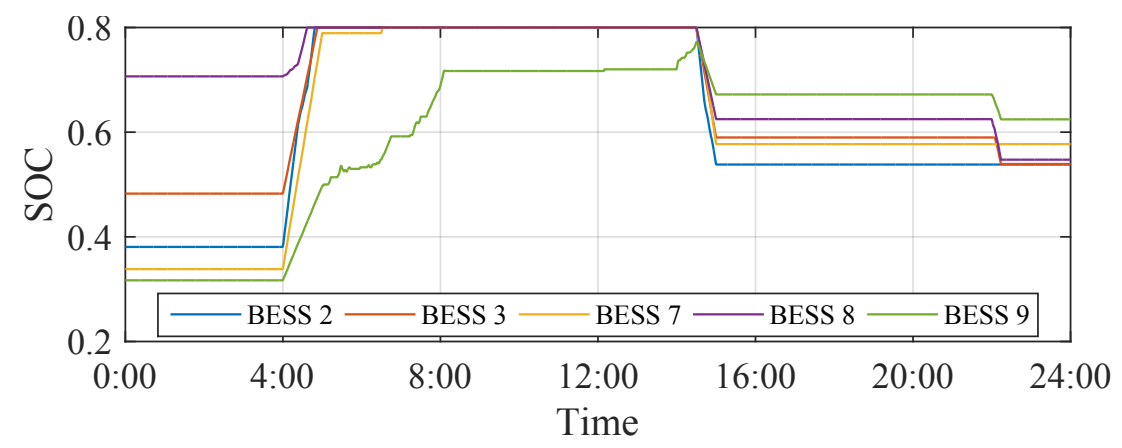

(b) SOC curves of Lithium-ion BESSs (L group).

Figure 5.9: Simulation results for the 24-hour period.

are shown in (5.19) and (5.20):

$$
\begin{gathered}
R_{C}=\lambda_{\text {reg }} \cdot P_{B E S S} \cdot R E G_{C C P} \cdot S \\
R_{P}=\lambda_{\text {reg }} \cdot P_{B E S S} \cdot \text { Mileage Ratio } \cdot R E G_{P C P} \cdot S
\end{gathered}
$$

where $R E G_{C C P}$ is the capacity clearing price for regulation in $\$ / \mathrm{MWh}$ and $R E G_{P C P}$ is the performance clearing price for regulation in $\$ / \mathrm{MW}$. Mileage Ratio represents the ratio between the requested mileage for the assigned resource (traditional or dynamic) to that of the traditional resources. Mileage Ratio further awards the dynamic resource for tracking longer mileage.

The influence of the regulation participation rate on CPI is studied and recorded in Fig. 5.10(a). According to the regulation income formulation (5.19) and (5.20), increasing power participation rate can boost the regulation market income greatly, but the income is also restricted by S. From $\lambda_{\text {reg }}=0.1$ to $\lambda_{\text {reg }}=0.5$, the CPI increases 


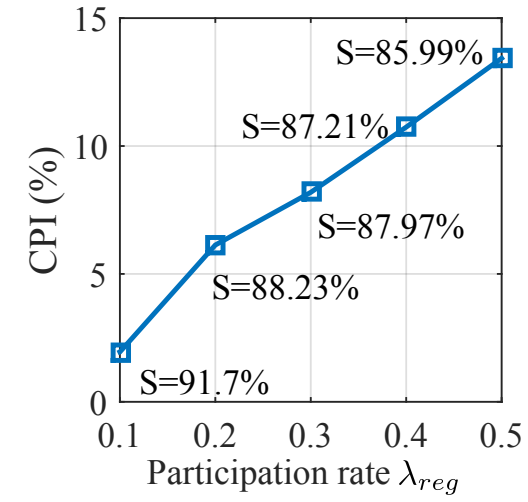

(a) CPI with different regulation participation.

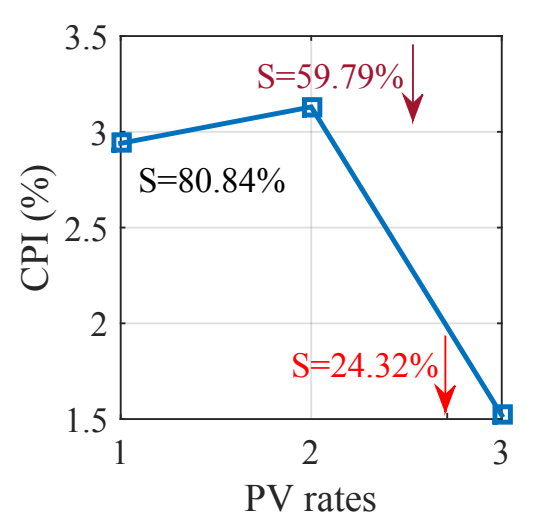

(b) CPI with different PV penetration.

Figure 5.10: CPI scores with varying regulation regulation participation rates and PV penetration rates.

7 times from $1.95 \%$ to $13.43 \%$. However, when the participation rate increases further beyond 0.5 , the regulation score falls below the acceptable limit $40 \%$ [45], below which the entity will be disqualified from participating in regulation.

The impact of PV integration is also simulated. The resulting CPI scores are plotted in Fig. 5.10(b). With PV integration, the CPI will be improved due to the PV power output, whose cost is very low. However, when the penetration rate grows to 3 times the original setting, the BESSs are no longer able to deal with the fluctuations. As a result, the system cannot perform frequency regulation normally, and this leads to a very low performance score.

\subsection{Comparisons}

\subsubsection{Optimal Scheduler Comparison}

The proposed hierarchical controller scheme is neither fully centralized nor fully decentralized. BESSs are separated into groups in the Schedule Optimizer. Comparisons with a fully centralized controller are conducted through new simulations. Price data for one year is used in the simulations. In a fully centralized controller, each battery schedule is decided respectively instead of the group schedule. The results are listed in Table 5.4. The centralized controller offers the optimal schedule for each BESS, thus resulting in higher estimated annual revenue than the HiEMS. But the HiEMS is com- 
Table 5.4: Comparisons with a fully centralized scheduler

\begin{tabular}{|c|c|c|}
\hline Controller types & Estimated annual revenue & Average calculation time \\
\hline \hline Hierarchical & $\$ 35,180$ & $0.03-1 \mathrm{sec}$ \\
\hline Centralized & $\$ 35,501$ & $0.5-1.5 \mathrm{sec}$ \\
\hline
\end{tabular}

putationally more effective. It is also more efficient with less communication hardware setup cost, since communication is required between HiEMS and each aggregator but not between HiEMS and each BESS. Thus the proposed HiEMS is easier for a large scale implementation when more BESSs are participating.

\subsubsection{Real-time Performance Comparisons}

In this section, the HiEMS control performance is compared with the participation factor method (PF) and the master-slave method (M-S). The test signal is the summation of the overall BESS set point, real-time load fluctuations, and the dynamic regulation signal, taken from the base case study in Section 5.6.

The PF method [18] distributes the test signal to each BESS through its participation factor. Each participation factor is defined as the ratio of the individual BESS power rating to the total BESS power rating.

In the M-S method [17], the master BESS handles most of the commands, and the slave BESSs will only respond when the master reaches its SOC limit. The BESSs with the largest power ratings in group $\mathrm{V}$ and $\mathrm{L}$ are set as the masters in each group. The priority sequence in the $\mathrm{V}$ group is BESS $1>5>4>6$, with BESS 1 as the master; and the sequence in the L group is BESS $2>7>3>8>9$, with BESS 2 as the master. In all the controllers, one BESS will cease to respond when its SOC exceeds the permitted range.

\subsubsection{Performance Comparisons}

According to Fig. 5.8, there are two major charging events from 3:30 to 7:00, starting at 3:30 and 6:30 respectively. With a major energy arbitrage event and regulation service happening at the same time, BESS operation is critical to the overall performance. Fig. 


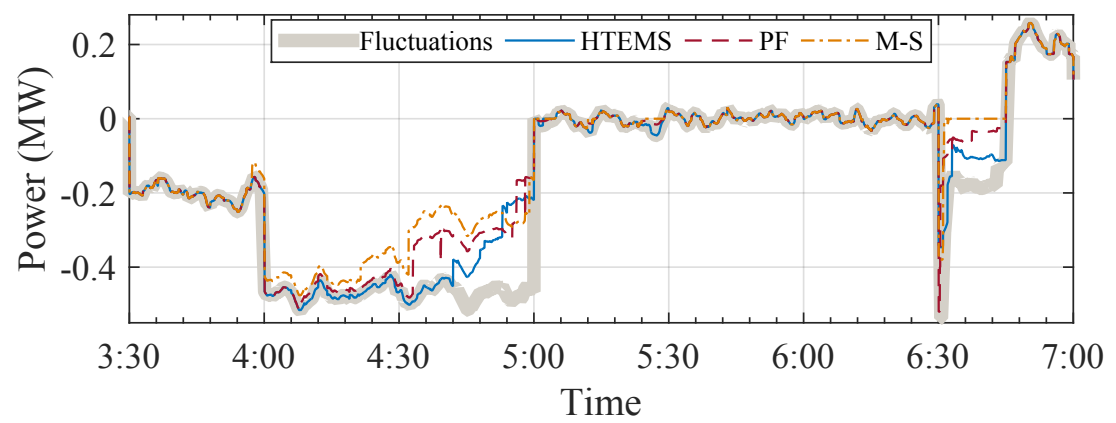

(a) Test signal and BESS outputs for three controllers

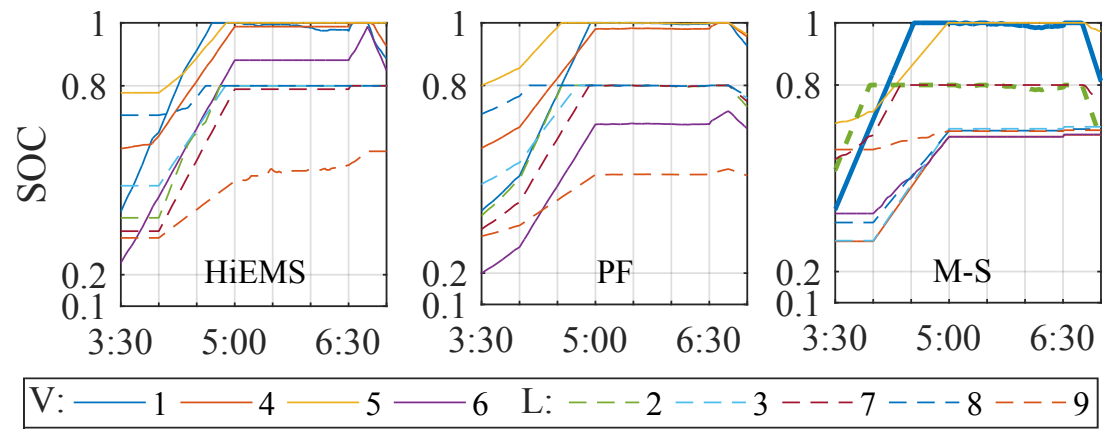

(b) SOC curves

Figure 5.11: Controller output and respective BESS SOC curves from 3:30 to 7:00.

5.11(a) shows the command and responses, and Fig. 5.11(b) records the SOC curves for the three controllers during this period.

Due to its SOC management function, the available BESS power of HiEMS is always larger than those of PF and M-S. For example, till 4:30, and all BESSs are still within SOC limits for HiEMS, and hence all the BESSs are available; in PF, BESS 8 reached its upper bound before 4:30, and thus $20 \mathrm{~kW}$ is unavailable; and in M-S, BESS 1, 2 and 7 reached upper bounds before 4:30, $200 \mathrm{~kW}$ is therefore unavailable. The SOC coordination has also been observed at another charging event at 6:30. Shortly after 6:30, the SOC of large BESSs are actually very close to saturation for all three controllers. However, HiEMS dynamically changes its Optimal Dispatch Plan to fully utilize the energy in small BESSs. Those small BESSs still have small participation factors in PF or low priority in M-S, such as BESS 6 and 9, whose SOC curves ramp up much faster in HiEMS than those in the other two controllers.

As concluded in Table 5.5, throughout the simulation day, HiEMS has only 2 hours of low performance $(\mathrm{S}<40 \%)$, while PF has 6 hours and M-S has 9 hours. As a result, 


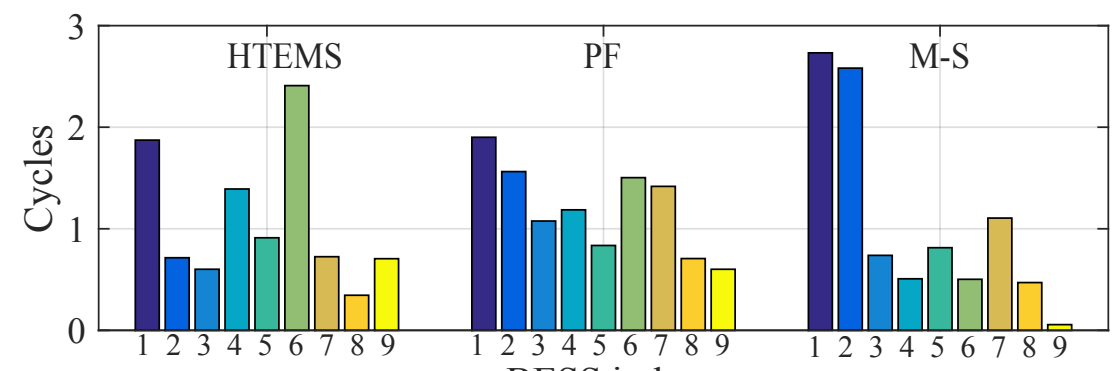

BESS index

Figure 5.12: Individual BESS equivalent usage cycles for three controllers.

Table 5.5: Comparisons between three control methods

\begin{tabular}{|c|c|c|c|c|}
\hline \multirow{2}{*}{\multicolumn{2}{|c|}{ Criteria }} & \multicolumn{3}{|c|}{ Controller schemes } \\
\hline & & HiEMS & $\mathrm{PF}$ & M-S \\
\hline \multicolumn{2}{|c|}{ Low performance hours } & 2 & 6 & 9 \\
\hline \multicolumn{2}{|c|}{ Average score (\%) } & 91.7 & 73.7 & 65.5 \\
\hline \multirow{2}{*}{$\begin{array}{l}\text { Average equivalent } \\
\text { usage cycles }\end{array}$} & $\overline{\mathrm{V} \text { group }}$ & 1.65 & 1.36 & 1.14 \\
\hline & L group & 0.62 & 1.07 & 0.99 \\
\hline \multicolumn{2}{|c|}{ Time of first replacement (years) } & 4.53 & 2.10 & 1.27 \\
\hline
\end{tabular}

the average performance score of HiEMS is $91.7 \%, 1.24$ times higher than $73.7 \%$ by PF, and 1.4 times higher than $65.5 \%$ by M-S.

\subsubsection{BESS Usage and Lifetime}

The equivalent usage cycles (UC) of the BESSs during the simulated time period is calculated by the formula in (5.1). The results are plotted in Fig. 5.12.

HiEMS uses cheaper BESSs more frequently, and thus limiting the L group usage due to their high costs. In comparison to HiEMS, PF uses larger BESSs more often. For instance, the UC values of BESS 2 and 7, which have the largest power rating (40 $\mathrm{kW}$ ) in the L group, are around 2 times larger than those of HiEMS. The UC of BESS 2 increases from 0.71 to 1.56 cycles, and that of BESS 7 increases from 0.72 to 1.41 cycles. In general, the average UC of the L group rises from 0.62 to 1.07 cycles, while that of the $\mathrm{V}$ group drops from 1.65 to 1.36 cycles, as recorded in Table 5.5.

For M-S, the master BESSs are over-burdened. The equivalent UC for BESS 1 is 2.73 cycles, while that of HiEMS and PF is 1.8 and 1.9 cycles respectively. Similarly, the equivalent UC for BESS 2 is 2.58 cycles, which is 3.6 times larger than 0.71 cycles 
by HiEMS and 1.65 times larger than 1.56 cycles by PF.

In practice, the minimum lifetime represents the first time when replacement is needed. The lifetime is estimated using the formulation in (5.2). The battery life cycle is chosen as 10,000 for the $\mathrm{V}$ group, and 1,200 for the $\mathrm{L}$ group [13]. The capacity loss caused by self-discharge and internal leakage is ignored. As recorded in Table 5.5, the first replacement time with HiEMS is 2.15 times longer than that of PF, and 3.57 times longer than that of M-S.

\subsection{Summary}

In this chapter, a HiEMS is proposed for multi-use distributed BESSs to improve their cost-effectiveness. Several BESSs can be aggregated and coordinated to participate in both the energy market and the regulation market. Multiple objectives are incorporated into two optimizers, both utilizing the MPC strategy.

Regulation performance is guaranteed by the schedule level and the dispatch level. Regulation Assessment in the schedule level arranges sufficient regulation energy, by setting a performance constraint $\theta_{\text {reg }}$ based on historical regulation signal. The Dispatch Optimizer carries out the real-time coordination of the BESSs, and the SOC management. This helps to maintain the performance. The proposed HiEMS can support up to 0.5 regulation participation rate, which will boost the CPI by 7 times. In terms of performance, HiEMS scores 91.7\%, outperforming PF by 1.24 times and M-S by 1.4 times.

Cost awareness of the BESSs helps HiEMS manage BESS usage, and postpone the replacement investment. DAC is included as a part of the costs in both optimizers. This ensures that the control commands are distributed to BESSs according to their specific battery characteristics and DOD conditions. As a result, the time of first replacement for HiEMS is 2.15 times larger than that of the PF method and 3.75 times larger than that of the master-slave method. 


\section{Chapter 6}

\section{BESS Scheduling in}

\section{Performance-based Regulation}

In Chapter 5, a HiEMS is proposed to aggregate multiple BESSs, and to achieve multi-market business operations. In Chapter 6, a dynamic scheduling strategy is developed to optimize the bids in the performance-based regulation market in PJM. By taking part in the performance-based regulation market, the CPI of the BESS aggregation can be further improved.

\subsection{Introduction}

As discussed in Chapter 3, a more financially rewarding performance-based regulation service is increasingly considered by BESSs $[120,16]$. In [16], the amount of energy consumed by regulation is set as a constant with a large margin (15 minutes or 0.25 $\mathrm{MWh} / \mathrm{MW}$ ) to ensure the high performance (above 0.9 out of 1 ), while the actual energy requirement remains uncertain. In real market revenue accounting, the final regulation revenue is proportional to the performance as well as the power bids. Therefore, when scheduling the energy capacity of BESS, keeping a large energy margin will limit the amount of power that BESS can bid into the markets; in other words, there is a trade-off between the performance and the bids. A constant regulation energy schedule cannot fully utilize the BESS capacity and will fail to get the optimal reward. 


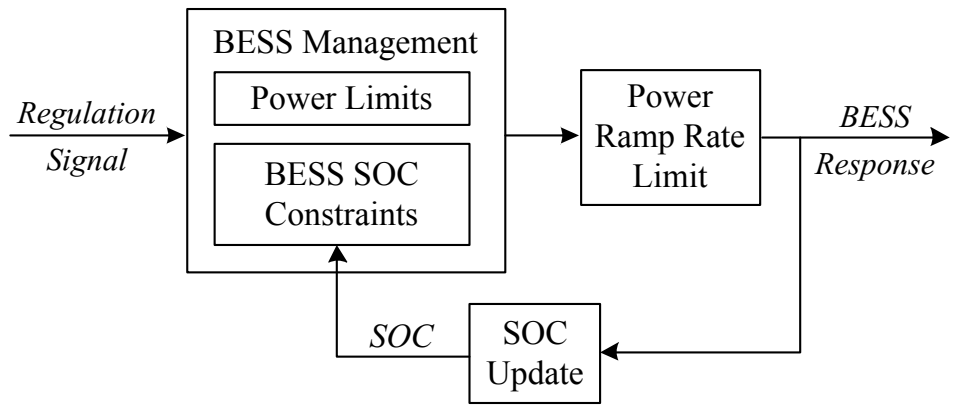

Figure 6.1: A general BESS model for response testing.

In this chapter, the optimal schedule optimizer includes an innovative realistic BESS performance model with regard to the regulation energy scheduling, which characterizes the trade-off between the performance and the power bids. The schedule optimizer also models the pricing uncertainties in both markets according to their respective statistical characteristics. The CPI of BESSs is analyzed to provide a reference for BESS entities to decide the optimal sizing and market participation to maximize the income of the multi-use BESSs.

The rest of this chapter is organized as follows: Section 6.2 introduces the simulation modeling and work flow of the regulation simulations, and discusses the performance results. Section 6.3 presents the problem formulation of the optimal scheduling in performance-based regulation market. Section 6.4 includes the simulation studies and analytical comparisons. Section 6.5 concludes the chapter.

\subsection{Regulation Simulations}

\subsubsection{BESS Modeling}

A general BESS model is developed as plotted in Fig. 6.1 to simulate the real time response to regulation signals. The regulation signal will be processed by a rule-based BESS management system [120] constraining the power and SOC within the limits shown in (6.1) and (6.2):

Power limits:

$$
P_{E}^{c, \max } \leq P(t) \leq P_{E}^{d, \max }
$$


SOC limits:

$$
S O C_{\min } \leq S O C(t) \leq S O C_{\max }
$$

where $P_{E}^{d, \max }$ is the maximum discharge rate; and $P_{E}^{c, \max }$ is the maximum charge rate.

If $P(t)$ exceeds/falls below the power limits, the response power will be set as $P_{E}^{d, m a x}$ or $P_{E}^{c, \max }$. If the state of charge $S O C(t)$ is greater than or equal to 0.8, the BESS cannot be charged anymore. If $S O C(t)$ is less than or equal to 0.2, the BESS cannot be discharged anymore.

The BESS response is further limited by the ramp rate. The chemical reactions in the battery can react within milliseconds. Therefore the power converters operation limit is the main ramp rate limiter in a BESS.

A SOC update block is also used to simulate the SOC value as the feedback to the BESS management system. The SOC calculation is based on the simple coulomb counting method in (6.3):

$$
S O C(t+1)=S O C(t)-\frac{P(t) \cdot \Delta t}{E_{B E S S}}
$$

where $E_{B E S S}$ is the rated BESS capacity; and $\Delta t$ is the duration time of each interval.

\subsubsection{Performance Score Simulations}

Performance score simulations are conducted using the BESS model and the historical real time operation regulation signal available at the PJM website [121]. The simulation procedure is described in Fig. 6.2. The simulations are conducted for different power ratings and capacity ratings. Given the same power rating, performance scores for each capacity setting is calculated according to (2.9) to (2.13).

The average scores and the respective score distribution ranges are plotted in Fig. 6.3. With the increase of the BESS energy capacity (MWh/MW), the distribution range gets smaller. This means that the probability for the BESS to saturate or deplete is less, and thus the risk of getting a low score decreases. Besides, the averages are mostly located in the higher score ranges rather than in the middle of the ranges. Normally BESS will perform very well in a high-score range, and the chances to get low scores 


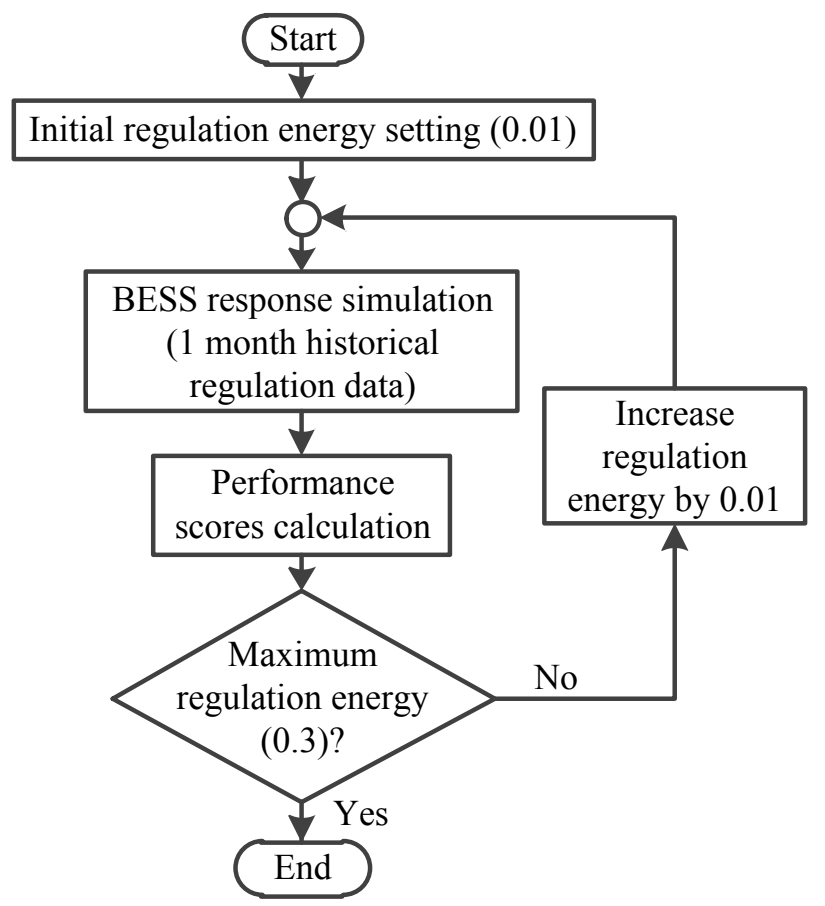

Figure 6.2: Work flow for performance score simulations.

are comparatively rare. However, after the energy capacity increases further above 0.25 $\mathrm{MWh} / \mathrm{MW}$, the average performance scores stay on similar levels. In other words, for a BESS with a 1 unit power rating, 0.25 unit hour energy capacity is totally sufficient for the high score performance for regulation market participation.

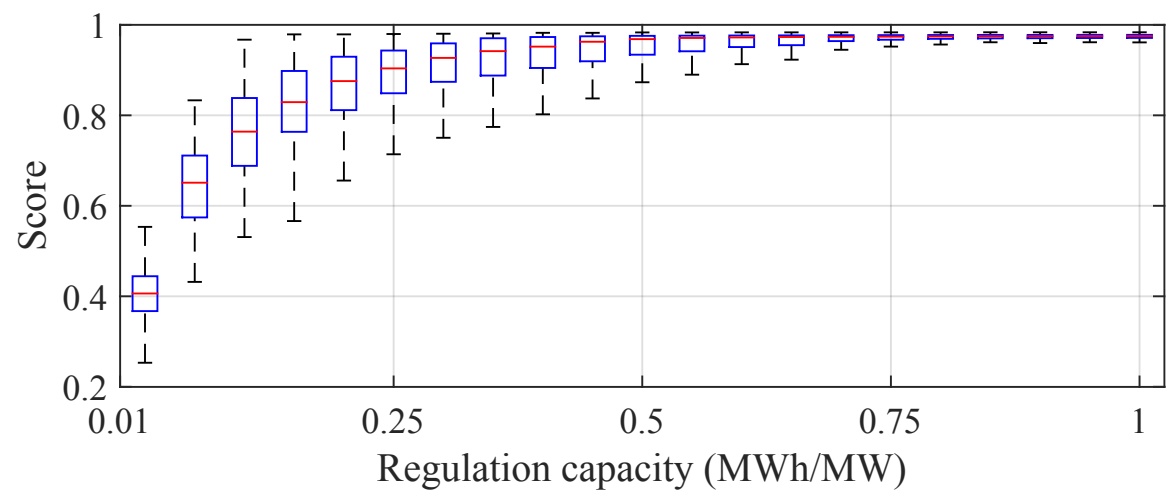

Figure 6.3: Performance scores for different regulation capacity settings. 


\subsection{Optimal Scheduling Formulation}

The scheduling objective function $V$ aims to schedule the BESS aggregators to maximize the overall profit, which consists of the three parts: electricity bill from the energy market, revenue from the regulation market, and the BESS usage costs. The objective function is defined in (6.4) as follows:

$$
\begin{aligned}
V:=\min _{P_{i, t}^{e, t}, P_{i, t}^{e, c}, P_{i, t}^{r}} \sum_{t}^{H_{p}}\left\{\sum_{s} p^{s}[\underbrace{c_{e, t}^{s} \bar{P}_{t}^{g r i d} h_{e}}_{\text {Energy market bill }}-\underbrace{\text { Income }}_{\text {Regulation revenue }} \text { reg,t }^{s}]\right. \\
+\sum_{i}^{n} \underbrace{\left[c_{b a t, i}\left(\frac{P_{i, t}^{e, c}+P_{i, t}^{e, d}}{2} h_{e}+P_{i, t}^{r} \bar{h}\right)\right]}_{\text {Battery cost }}\}
\end{aligned}
$$

where Income ${ }_{\text {reg,t }}^{s}$ represents the regulation income at time $t$ in scenario $s, \frac{P_{i, t}^{e, c}+P_{i, t}^{e, d}}{2} h_{e}$ stands for the planned equivalent usage cycle for each group, and $\bar{h}$ represents the hourly average energy consumption (0.13 MWh/MW) by dynamic regulation service.

The regulation income has two components as shown in (6.5): $R C$ in (6.6) and $R P$ in (6.7). $R C$ is the capacity revenue for reserving the capacity, and $R P$ is the performance revenue.

$$
\text { Income }_{\mathrm{reg}, \mathrm{t}}^{s}=R C_{t}^{s}+R P_{t}^{s}
$$

where

$$
\begin{gathered}
R C_{t}^{s}=c p_{t}^{s} \cdot S\left(h_{t}\right) \cdot \sum_{i} P_{i, t}^{r} \\
R P_{t}^{s}=p p_{t}^{s} \cdot S\left(h_{t}\right) \cdot \mathrm{MR} \cdot \sum_{i} P_{i, t}^{r}
\end{gathered}
$$

where $S\left(h_{t}\right)$ is the performance model, and MR represents the ratio between the requested mileage for the assigned resource (traditional or dynamic) to that of the traditional resources. MR further awards the dynamic resource for tracking longer mileage.

While BESS performance is usually assumed to be constantly high, the energy con- 
sumption by regulation service actually has an effect on the BESS score. This chapter formulates a dynamic performance $S\left(h_{t}\right)$ with regard to the regulation energy $h_{t}$ for the regulation market.

The dynamic performance can be piecewise linearized as in (6.8).

$$
S\left(h_{t}\right)=\sum_{m}\left(a_{m} \cdot h_{m, t}+b_{m} \cdot U_{m, t}\right)
$$

where $h_{t}=\sum_{m} h_{m, t}$.

The objective function is subject to the constraints discussed below.

\section{Power limits from power rating and bid constraints}

Equation (6.9) represents the power rating limit for the BESS groups. The power range for regulation will always be set as standby and not accessible for energy arbitrage.

$$
\begin{gathered}
P_{i}^{\min }+P_{i, t}^{r} \leq P_{i, t}^{e, c}+P_{i, t}^{e, d} \leq P_{i}^{\max }-P_{i, t}^{r} \quad \forall i, t \\
P_{i, t}^{r} \geq 0 \quad \forall i, t
\end{gathered}
$$

\section{Power balance constraint}

$$
\bar{P}_{t}^{\text {grid }}=\bar{P}_{t}^{\text {load }}-\bar{P}_{t}^{P V}-\sum_{i}\left(P_{i, t}^{e, d}-P_{i, t}^{e, c}\right)
$$

\section{Energy constraints}

The average energy stored of each group is predicted by coulomb-counting method [103] shown in (6.12), considering different charging and discharging efficiencies $\eta_{i}^{d}$ and 
$\eta_{i}^{c}$ for various battery groups.

$$
E_{i, t}=E_{i, t-1}-\underbrace{\frac{1}{\eta_{i}^{d}} \cdot P_{i, t}^{e, d}+\eta_{i}^{c} \cdot P_{i, t}^{e, c}}_{\text {Energy arbitrage }}-\underbrace{\frac{\bar{h}}{\eta_{i}^{d}} \cdot P_{i, t}^{r}+\eta_{i}^{c} \cdot \bar{h} \cdot P_{i, t}^{r}}_{\text {Regulation energy consumption }}
$$

For each hour, the remained energy should be sufficient to maintain the schedule performance for providing both regulation up and down.

$$
E_{i}^{\min }+P_{i, t}^{r} \sum_{m} h_{m, t} \leq E_{i, t} \leq E_{i}^{\max }-P_{i, t}^{r} \sum_{m} h_{m, t} \quad \forall i, t
$$

To ensure continuous normal operation, the final energy stored is constrained to a certain value. In the simulation studies, the final energy constraint is set as $50 \%$ at the 24th hour.

$$
E_{i, 24}=0.5 E_{i}^{\max } \quad \forall i
$$

\section{Discharge cycle constraints}

The daily equivalent discharge cycle can be constrained to limit the usage of the battery as in (6.15) [83], where $\gamma$ is the number of allowed discharge cycles. A larger $\gamma$ allows more market participation, while a lower $\gamma$ restricts BESS bids. A proper $\gamma$ value can balance the economic profit and the BESS degradation.

$$
\sum_{t}^{24}\left[\frac{P_{i, t}^{e, c h}+P_{i, t}^{e, d}}{2}+P_{i, t}^{r} \sum_{m} h_{m, t}\right] \leq \gamma\left(E_{i}^{\max }-E_{i}^{\min }\right)
$$

\section{Binary variable constraints}

Binary variable indicates whether the variable falls into a certain segment.

$$
\sum_{m} U_{m, t}=1 \quad \forall t
$$




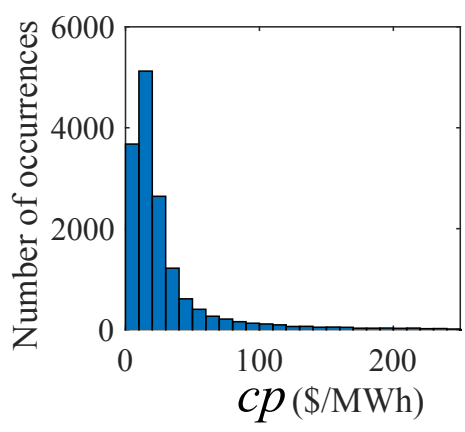

(a) Distribution of $c p$.

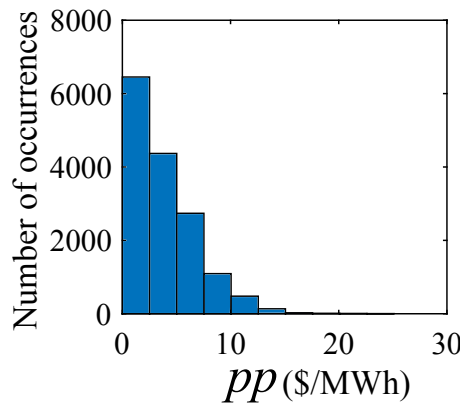

(b) Distribution of $p p$.

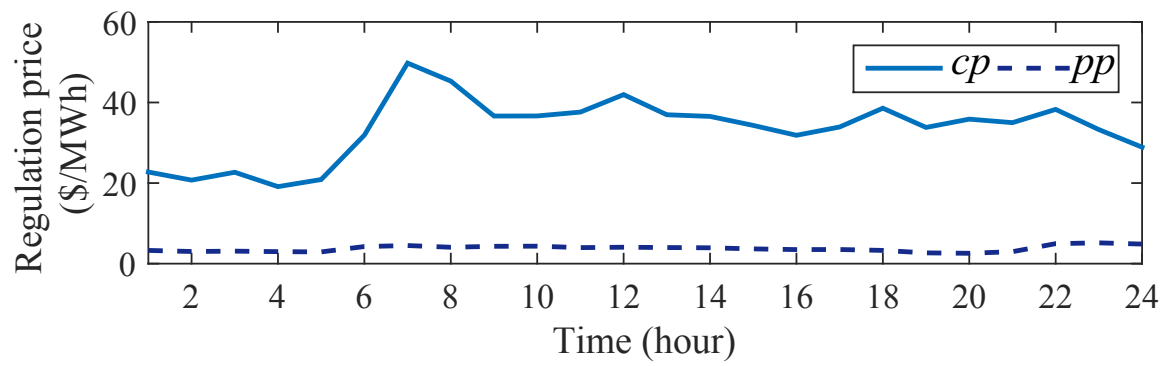

(c) Average regulation prices.

Figure 6.4: Regulation price distributions of a 638-day database.

\subsection{Simulation Studies}

\subsubsection{Scenario Generation}

The uncertainty of energy and regulation prices is represented by a set of scenarios and their respective probabilities of realization.

\section{Probability distribution}

Regulation prices are highly unpredictable, and thus the scenarios are generated based on historical data. The distributions are plotted in Fig. 6.4(a) and Fig. 6.4(b). The average prices among all scenarios are plotted in Fig. 6.4(c).

For the USEP, future prices can be predicted by an artificial neural network trained using historical data base. These point forecast results can be expanded to probability forecast using quantile regression [122]. The predicted prices with probabilities are shown in Fig. 6.5. The USEP is averaged to a 24 -hour time scheme in the optimizer, and the Singapore dollar is converted to the US dollar by an exchange rate of 1:0.72. 


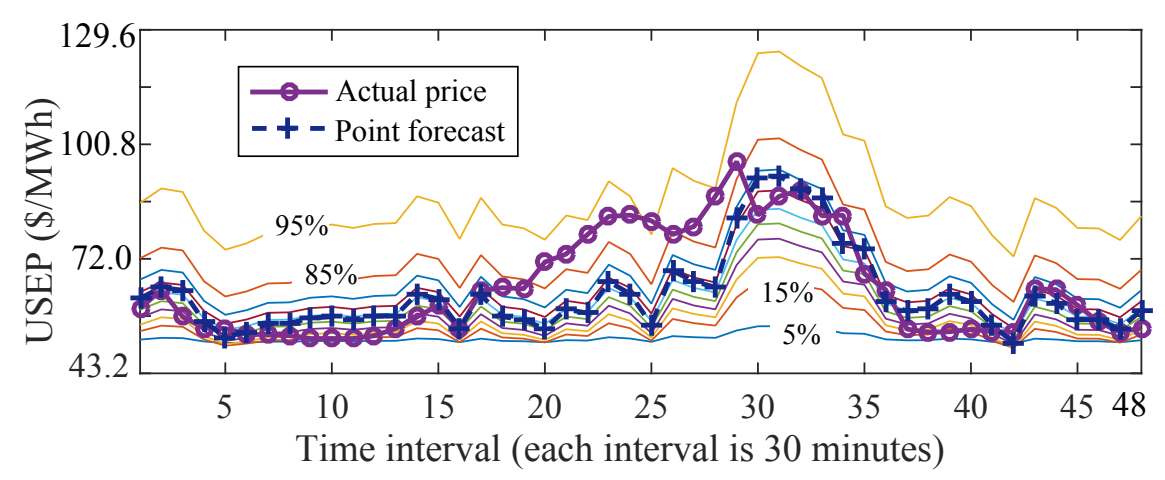

Figure 6.5: Probabilistic forecast results for USEP.

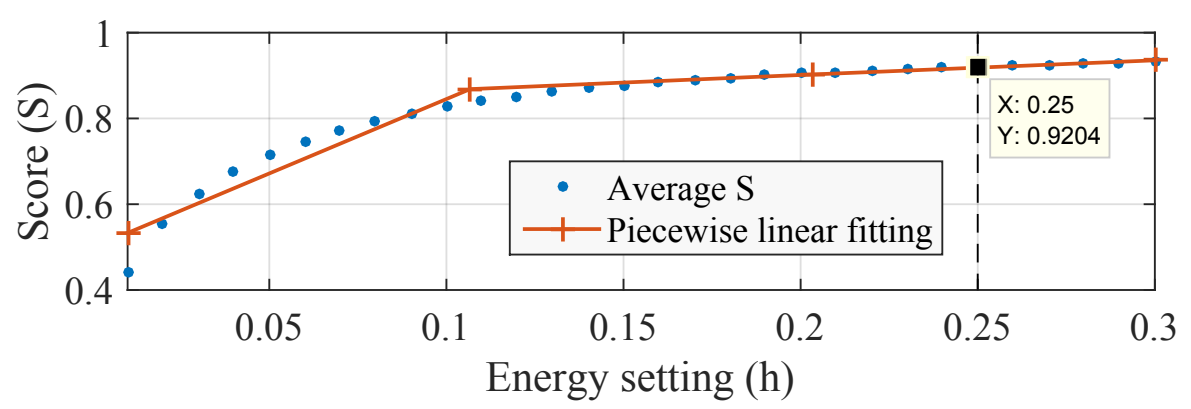

Figure 6.6: Dynamic performance and piecewise linear fitting results.

For regulation prices, a 638-day database is used to capture the distribution of the prices, and the price prediction is generated randomly from this database.

\section{Scenario generation and reduction}

The Monte Carlo method is utilized to generate a large number of scenarios [123]. Random possibilities between $[0,1]$ are separately generated for each parameter at each time interval, and the values are chosen according to the distributions generated above.

After generating desired number of scenarios, the probability of each scenario is normalized so that the total probabilities add up to 1 . To further lower the calculation burden, numbers of scenarios can be reduced using a scenario reduction package SCENRED in GAMS.

Discharge cycle constraint $\gamma$ is chosen as 2.5 for the $\mathrm{V}$ group and 1.4 for the $\mathrm{L}$ group. The dynamic performance is studied based on historical database and piecewise linearized. 


\subsubsection{Base Case and Comparisons}

\section{Scheduling results}

The schedule optimizer results are shown in Fig. 6.7. The result in Fig. 6.7(a) is compared with the result of a constant optimizer using a constant regulation energy $h=0.25 \mathrm{MWh} / \mathrm{MW}$ and score $S=0.92$, as shown in Fig. 6.7(b). The estimated state of charge curves are also recorded in Fig. 6.7(a) and Fig. 6.7(b). The dynamic regulation energy schedule $h_{t}$ and the respective expected scores $S$ are plotted in Fig. 6.7(c).

For both optimizers, the EMS bids most of its power into the regulation market for most of the time. Charging activities are minimized only to maintain a sufficient SOC level. Lithium batteries are generally used less due to their high cost.

When compared to Fig. 6.7(b), the proposed dynamic optimizer in Fig. 6.7(a) manages to bid more power into regulation market, resulting from a more realistic and flexible regulation energy schedule. There are many hours when the dynamic optimizer is able to bid full power, while the constant optimizer can no longer provide power bidding because of stricter energy constraints such as hour 16 and 17 .

In some intervals in Fig. 6.7(c), the regulation energy $h_{t}$ is less than the typical value of 0.25 . Although the resulting expected $S$ will be lower than the optimal value of 0.92 , the dynamic optimizer manages to boost the regulation income from more power bids, such as in hour 6,7 , and 8 . Conversely, in hour 3,4 , and 5, the optimizer makes up for small power bids by reserving more $h_{t}$.

\section{Real-time performance tests}

The optimal scheduling strategy was implemented in the HiEMS in Chapter 5. The SOC curves from a 24-hour test are plotted in Fig. 6.8. In the particular tested day, the actual regulation energy is even smaller than the dynamically scheduled regulation energy. As a result, the $\mathrm{V}$ group is already sufficient in responding the regulation signal, while the L group is often not utilized.

Comparisons with PF and M-S are also conducted. The updated HiEMS scores 0.92, outperforms PF by 1.02 times and M-S by 1.12 times. The time of first replacement for HiEMS is 2.92 times larger than that of the PF method and 7.56 times larger than that of the master-slave method. 


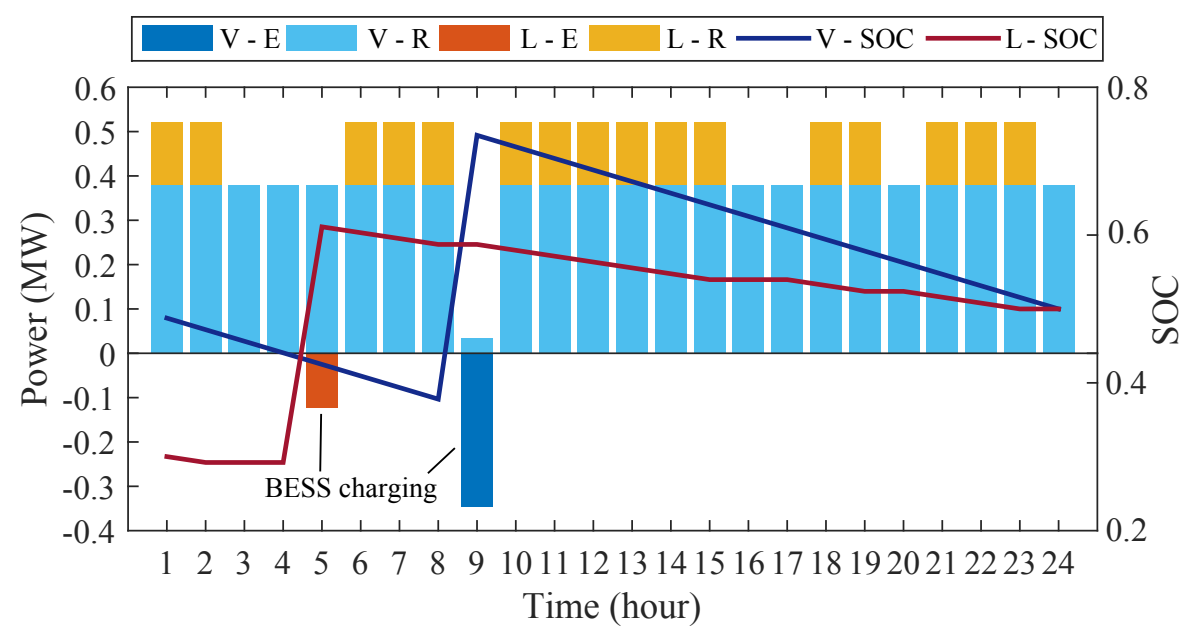

(a) Schedule Optimizer results (E stands for Energy market, R stands for regulation market).

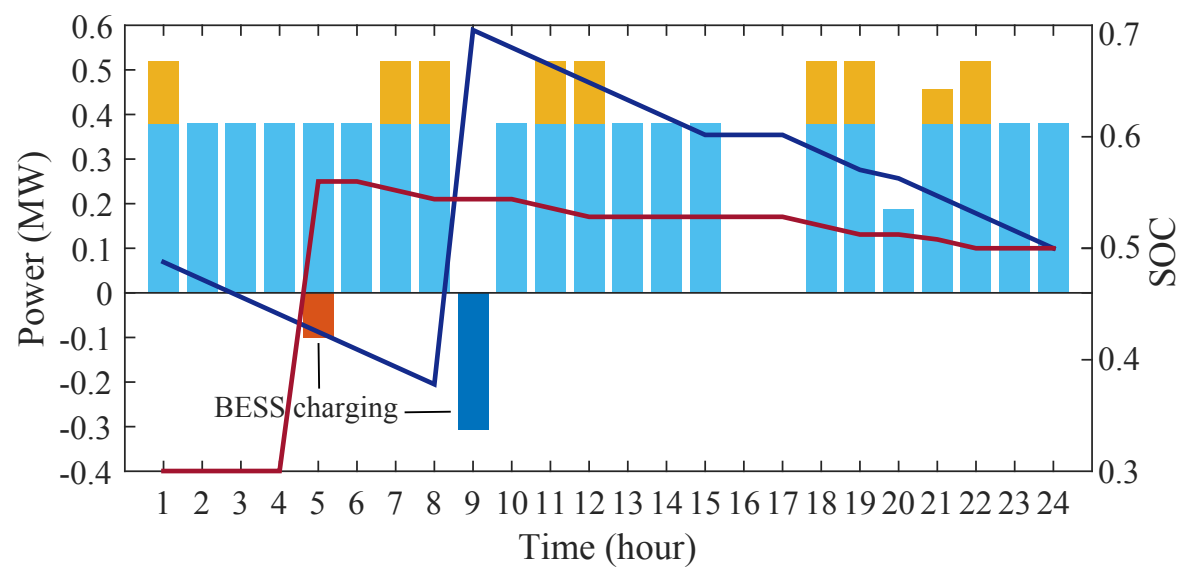

(b) Schedule Optimizer results with constant score.

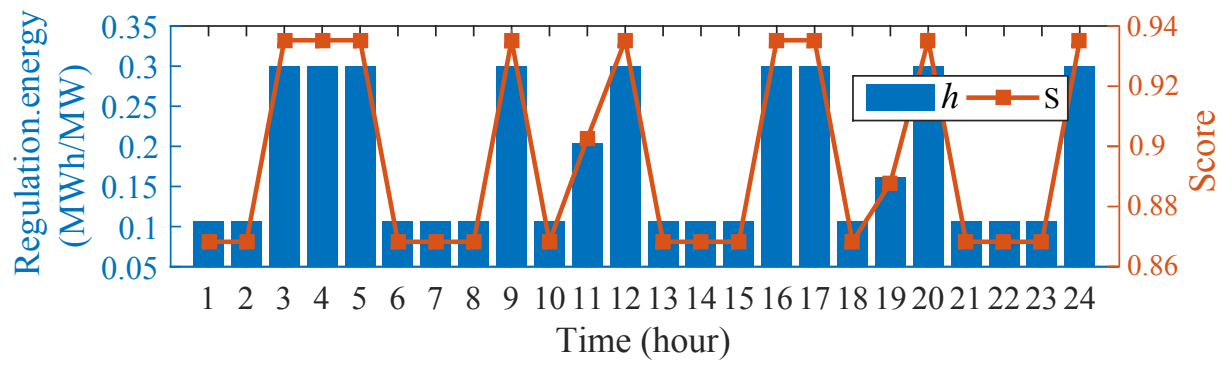

(c) Regulation energy schedule and expected performance scores.

Figure 6.7: 24-hour schedules with dynamic performance and constant score. 


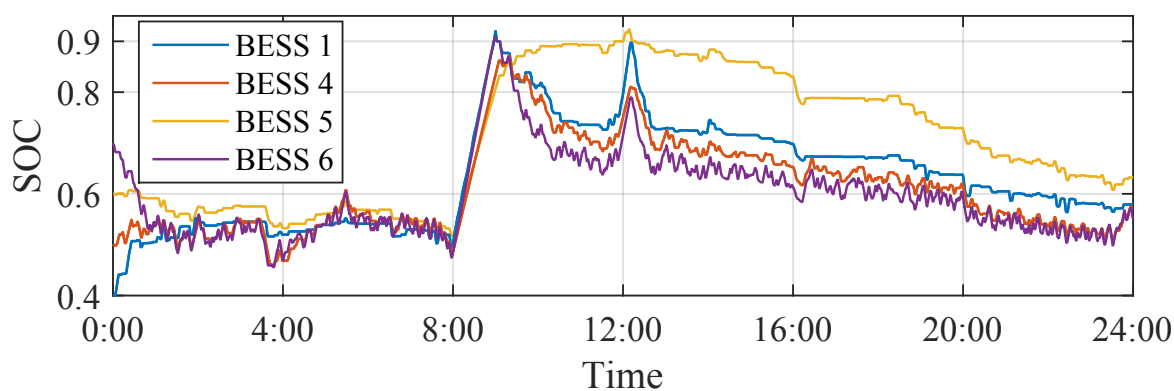

(a) V group SOC curves.

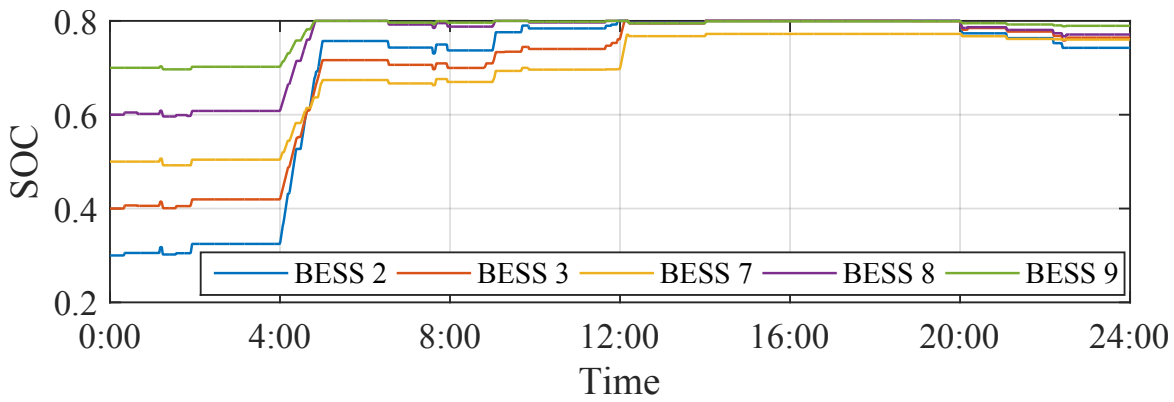

(b) L group SOC curves.

Figure 6.8: 24-hour simulation SOC results.

Table 6.1: Comparisons between dynamic and constant score

\begin{tabular}{|c|c|c|c|}
\hline \multicolumn{2}{|c|}{} & Dynamic & Constant \\
\hline \multicolumn{2}{|c|}{ Energy income (\$/day) } & -34.56 & -30.02 \\
\hline \multirow{2}{*}{$\begin{array}{c}\text { Regulation } \\
\text { income (\$/day) })\end{array}$} & Capacity & 342.13 & 280.79 \\
\cline { 2 - 4 } & Performance & 114.11 & 93.81 \\
\hline \multicolumn{2}{|c|}{ Total daily income (\$/day) } & 421.68 & 344.57 \\
\hline \hline \multicolumn{2}{|c|}{ Total DAC (\$/year) } & 383,155 & 394,710 \\
\hline \hline \multicolumn{2}{|c|}{ CPI } & $40.17 \%$ & $31.88 \%$ \\
\hline
\end{tabular}

\subsubsection{Income Analysis}

A detailed income breakdown is shown in Table 6.1. While the dynamic optimizer pays more electricity bill for charging, it earns more revenue from the regulation market, and ends up with a higher overall daily income.

To further study the cost-effectiveness, cost-performance index (CPI) is calculated in Table 6.1. The total earned value is estimated by multiplying the daily income by 365 days. The total actual cost for the BESSs is calculated based on the same sets of BESS 
Table 6.2: Comparisons between different scenario numbers for a 238-day period

\begin{tabular}{|c|c|c|c|}
\hline \multicolumn{2}{|c|}{ Price information } & Actual income (\$) & Regret (\$) \\
\hline \multicolumn{2}{|c|}{ Perfect prediction } & 81,609 & N.A. \\
\hline \multirow{3}{*}{ Number of scenarios } & 10 & 75,888 & $-5,721$ \\
\cline { 2 - 4 } & 50 & 75,954 & $-5,655$ \\
\cline { 2 - 4 } & 100 & 78,383 & $-3,226$ \\
\hline
\end{tabular}

groups used in Table 5.1, Chapter 5.

By participating in the regulation market, BESS entities can greatly increase the CPI, while their CPI ranges from $4 \%$ to $15 \%$ when only providing energy arbitrage [14]. By dynamically adjusting the regulation energy schedule, the CPI can be further boosted to $40.17 \%$.

\subsubsection{Impacts of Scenario Numbers}

To study the long term effect of the scenario-based optimization, scheduling tests are carried out for a 238-day period with varying numbers of scenarios. For each day, required price scenarios are generated and used in the schedule optimizer. The real income of the calculated schedules is assessed with the actual price on a certain day. As a comparison, the perfect prediction case uses the actual prices in the optimizer, indicating that no forecast error is involved. The regret value is defined as the difference between the actual income and the income with perfect prediction.

According to the results summarized in Table 6.2, optimization with more scenarios has a better representation of the possible price distribution, which is more robust in gaining a better income over a long period of time. However, more scenarios will greatly increase the computation burden. Therefore, a proper number of scenarios can be chosen considering the allowed computation time and the entity's risk tolerance.

\subsubsection{Impact of PV Penetration}

The impact of PV integration is simulated with different penetration settings. The original setting refers to $67.5 \%$ of the peak load power. The resulting CPI and score values are plotted in Fig. 6.9. With increased PV penetration, the CPI will be improved 


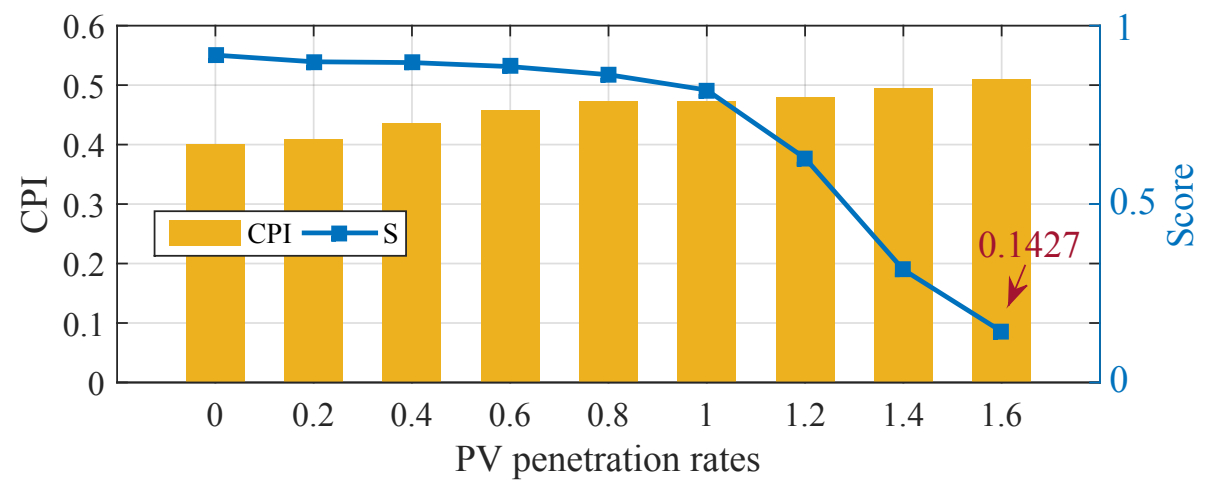

Figure 6.9: CPI and score for different PV penetration settings.

due to the PV power output. However, when the penetration rate grows to 1.6 times the original setting, the BESSs are not able to deal with the fluctuations. The system frequency regulation performance drops to 0.1427 . According to market rules, the entity will not be rewarded for that hour when the hourly score is lower than 0.25 [124]. Furthermore, the regulation entity will be disqualified if its 100-hour historical score drops below 40\% [45]. Therefore, the maximum CPI for this system can be boosted to no larger than $51.11 \%$ with PV generation.

\subsection{Summary}

This chapter proposes an optimal scheduling strategy for BESSs participating in energy and performance-based regulation markets. Realistic regulation performance is modeled to guarantee the optimal regulation energy schedule, and thus gaining maximum profit.

The scheduling optimizer is also implemented in the HiEMS in Chapter 5. After bringing in the trade-off between the performance and power bids, the proposed scheduler boosts the CPI to $40.17 \%$, compared to around $10 \%$ from only energy market participation, and $31.88 \%$ with constant score assumption. In terms of real-time performance, the dispatch optimizer coordinates the BESSs and manages the SOC.

With regard to the real-time performance, the updated HiEMS scores 0.92, outperforms PF by 1.02 times and M-S by 1.12 times. The time of first replacement for HiEMS is 2.92 times larger than that of the PF method and 7.56 times larger than that of the master-slave method. 
Lastly, the CPI can be further improved by integration PV generation. However, the PV fluctuations will bring more challenge to the BESSs in maintaining an acceptable performance. In the tested system, the CPI maximum value can be no larger than $51.11 \%$ with increased PV generation. 


\section{Chapter 7}

\section{Conclusions and Future Works}

In this chapter, the background, objectives and importance of this research are recapitulated. Key outcomes from simulation studies are summarized and highlighted. Towards the end of this chapter, some possible future directions are suggested to extend this work.

\subsection{Conclusions}

\section{Research Outline}

The research works in this thesis set out to study the control and optimization issues regarding the penetration of BESSs in current power systems. As BESSs are very fast in response and flexible in control strategies, there have been many research works proposing various applications for BESSs. In this research, we focus on BESS applications for system frequency regulation. System frequency regulation issues are worthy of studying for both technical and economic reasons discussed below.

From the technical viewpoint, today's power system is having increasing numbers of DERs and RESs connected, especially in distribution networks. RESs bring about challenges for system frequency regulation, as a result of their intermittent power output and their non-sensitivity towards system frequency. However, traditional generation plants are less effective in responding to fast and drastic frequency changes. BESSs are good candidates to effectively stabilize system frequency within a short period of time. Therefore, an optimal sizing strategy and controller are required considering the 
characteristics of frequency control issue. For this, an optimal penetration rate of BESSs is studied in Chapter 3 given the current regulation control scheme. And the regulation controller is further improved by implementing the MPC algorithm in Chapter 4.

From the economical viewpoint, although the battery cost is decreasing due to the advancing technologies, the current BESSs are still very hard to gain profit, even given the optimal sizing strategy and control method. In such a circumstance, this thesis tries to maximize the BESS profit by taking part in multiple competitive electricity markets. Most studies are either focusing on the energy market only or working on multi-market participation with simplified models. Therefore, this thesis concentrates on multiple market participation, including real-time energy market and regulation market. For the market mechanism, we study the current most profitable performance-based regulation market in PJM, and include the realistic BESS performance into the BESS SOC update model to get an accurate prediction. The hierarchical EMS proposed is elaborated in Chapter 5 .

\section{Major Findings}

In Chapter 3, after carrying out iterative sizing studies based on long term system performance, an optimal penetration rate of BESSs in a system can be found. For our test system evaluated using the CPS criteria, the range of the best penetration rate is $5 \%$ to $15 \%$. In our simulation tests, the BESS aggregation can be easily controlled using the proposed decentralized control scheme. The proposed sizing strategy and control scheme can serve as a reference for future regulation market development. For example, firstly, the overall amount of scheduled regulation bids from BESSs is better kept within the recommended range, beyond which the system operator and the users will be paying a high price for BESS regulation while the system performance is not necessary greatly improved. Secondly, for big systems with large numbers of BESSs or other demandside regulation resources, a decentralized rule-based battery management system is very robust and effective, and easy for large scale expansion.

In Chapter 4, several resources are aggregated into a VPP, including BESS, CHP and PV. The VPP is utilized in system frequency control as well. Instead of a traditional PID scheme, the system load frequency control signal is generated by the centralized MPC controller. With the system dynamic model, the MPC controller is capable of increasing 
the system frequency dynamics, and considering the constraints from the regulation resource at the same time. Therefore, given a proper parameter identification method and a reliable communication network, the system frequency controller can be upgraded to improve the system performance and make good use of available resources.

Lastly, to improve the cost-effectiveness of BESS aggregations, a HiEMS for multimarket participation is introduced and tested in Chapter 5. Considering the realistic BESS performance model in Chapter 6, the proposed HiEMS is able to boost the CPI to $31.70 \%$ from around $10 \%$ by single market participation. The HiEMS also manages to coordinate the BESSs to achieve a better performance, scoring 0.92 in dynamic regulation. Moreover, the BESS usage is more balanced, contributing to the postponement of the first replacement investment. The time of first replacement for HiEMS is 2.92 times larger than that of the PF method and 7.56 times larger than that of the master-slave method. In real applications, the advantage of using such a HiEMS is that many features and coefficients are completely customizable. For example, the battery cost and revenue equilibrium is adjustable with regard to the planned battery lifetime; and the SOC management function can be stricter or less strict depending on customers' expectations. In short, the proposed HiEMS helps the entity to increase device awareness and improve the overall profitability in a flexible manner.

\subsection{Recommendations for Future Works}

\section{System Level Optimal Planning with BESS and PV}

In Subsection 3.4.2, the system CPS1 score is studied with different PV generation. As shown in Fig. 3.10, system performance declines greatly with the increase of PV penetration rate. Besides, the level of performance deterioration is varying with data from different days. Intuitively speaking, the system frequency can be recovered to the optimal performance with more BESS capacity, but the amount of BESS needed remains uncertain. This leads to a planning optimization issue for the system operator: what is the most recommended BESS penetration rate in the system? If considering the worstcase in the database, the resulting penetration rate will be conservatively large, which is redundant in most times and thus uneconomical. Conversely, optimistic optimiza- 
tion with best-case scenarios may result in severe frequency fluctuations in certain days. Therefore, the selection of uncertainty set is crucial for system level planning. Statistical studies of PV data and a better long-term PV prediction is needed to find the optimal BESS penetration rate.

\section{Improved Model-based System Control}

In Chapter 4, the proposed MPC controller is demonstrated with a simplified power system model, where each component is linearized and lower-order models are used to capture the short-term system dynamics. This can be effective for small systems like microgrids or small-scale one-area systems, but for large dynamic systems, the controller can be greatly improved in the following aspects:

- On-line system parameter identification: Parameters can be corrected in real-time to improve prediction accuracy and avoid severe control errors;

- MPC controller coupled with extend Kalman filter (EKF): Currently the system is linearized, while the real system is highly nonlinear and many parameters are very hard to measure. Therefore, EKF can be used to estimate the state variables using a nonlinear system model, which helps to better capture the real system dynamics.

- Battery management: The proposed MPC only the optimized BESS's power set point. The MPC can be further improved to include more detailed battery dynamics in the prediction model, and battery utilization cost in the objective functions. As a result, the battery management can be achieved in the system level MPC controller.

\section{Optimal Bidding Strategy for BESS Aggregation}

In most research works today, the BESS aggregation or VPP is regarded as a price taker, i.e. the optimization process relies on static pricing signals or price scenarios. However, in real system operations, when the BESS or VPP penetration rate increases to a certain level, their bidding will make a difference in the real-time market clearing process. This will lead to a difference in the final clearing price, and the BESS or VPP may fail to reach the planned revenue. Therefore, the market clearing model can be further included in the EMS to consider the market influence of the BESS or VPP. This can be done using a two-stage optimization scheme, where the BESS or VPP 
optimization and system level market clearing optimization are iteratively solved until a convergence within the preset tolerance is reached. The main difficulty in this includes:

- Market model building: Since participant data are normally confidential, it is very hard to capture the real market response given the BESS or VPP bids. Therefore, either the market model will be built based on some simplification, or Monte Carlo simulation methods or other artificial intelligence methods can be used to estimate the system response.

- Optimization method development: When dealing with large scale nonlinear optimization, the optimization problem may even turn out to be intractable and takes long time to solve. However, when participating in the real-time market, the calculation time of a participant is very limited. Therefore, an appropriate optimization algorithm can be improved to ensure the performance in limited time.

\section{VPP with Other Participants}

In this thesis, the VPP is only modeled with CHP and BESS, and the PV and loads are non-controllable. With the development of smart homes and increasing numbers of controllable devices, the VPP scheme can be further developed to include other resources, for example, heating, venting, and air conditioning system in most buildings. Similar to the studies in this thesis, the optimal amount of controllable devices can be studied, based on specific component characteristic and system performance. And a proper EMS can be developed to better coordinate various resources within the network.

In short, various research works on different aspects can be done to contribute to better planning, control, and coordination of the future grid, where multiple controllable resources can be integrated actively and play their roles in the power system. 


\section{References}

[1] (2016) Singapore EMA: Solar photovoltaic systems. [Online]. Available: https://www.ema.gov.sg/Solar_Photovoltaic_Systems.aspx

[2] "Kermit study report," DNV GL, Tech. Rep., 2012.

[3] N. Atic, A. Feliachi, and D. Rerkpreedapong, "Cps1 and cps2 compliant wedgeshaped model predictive load frequency control," in IEEE Power 86 Energy Society General Meeting, vol. 1, Jun 2004, pp. 855-860.

[4] I. Serban and C. Marinescu, "Control strategy of three-phase battery energy storage systems for frequency support in microgrids and with uninterrupted supply of local loads," IEEE Trans. Power Electron., vol. 29, no. 9, pp. 5010-5020, Sept 2014.

[5] S. Zhang, Y. Mishra, and G. Ledwich, "Battery energy storage systems to improve power system frequency response," in Power Engineering Conference (AUPEC), 2014 Australasian Universities, Sept 2014, pp. 1-5.

[6] Y. Cheng, M. Tabrizi, M. Sahni, A. Povedano, and D. Nichols, "Dynamic available agc based approach for enhancing utility scale energy storage performance," IEEE Trans. Smart Grid, vol. 5, no. 2, pp. 1070-1078, March 2014.

[7] T. Sasaki, T. Kadoya, and K. Enomoto, "Study on load frequency control using redox flow batteries," IEEE Trans. Power Syst., vol. 19, no. 1, pp. 660-667, Feb 2004 .

[8] (2017) Implementation and rationale for PJMs conditional neutrality regulation signals. [Online]. Available: http://www.pjm.com/ /media/committees-groups/ task-forces/rmistf/postings/regulation-market-whitepaper.ashx

[9] A. A. Hussein, N. Kutkut, Z. J. Shen, and I. Batarseh, "Distributed battery microstorage systems design and operation in a deregulated electricity market," IEEE Trans. Sustain. Energy, vol. 3, no. 3, pp. 545-556, July 2012.

[10] Y. Yang, H. Li, and A. Aichhorn, "Sizing strategy of distributed battery storage system with high penetration of photovoltaic for voltage regulation and peak load shaving," IEEE Trans. Smart Grid, vol. 5, no. 2, pp. 982-991, 2014.

[11] C. Blanc, "Modeling of a vanadium redox flow battery electricity storage system," Ph.D. dissertation, Ecole Polytechnique Fédérale de Lausanne, 2009. 
[12] H. Chen, T. N. Cong, W. Yang, C. Tan, Y. Li, and Y. Ding, "Progress in electrical energy storage system: A critical review," Progress in Natural Science, vol. 19, no. 3, pp. 291 - 312, 2009. [Online]. Available: http://www.sciencedirect.com/science/article/pii/S100200710800381X

[13] C. Menictas, M. Skyllas-Kazcos, and T. M. Lim, Eds., Advances in Batteries for Medium and Large-Scale Energy Storage. Woodhead Publishing, December 2014.

[14] J. M. Gantz, S. M. Amin, and A. M. Giacomoni, "Optimal capacity partitioning of multi-use customer-premise energy storage systems," IEEE Trans. Smart Grid, vol. 5, no. 3, pp. 1292-1299, May 2014.

[15] R. L. Fares, J. P. Meyers, and M. E. Webber, "A dynamic model-based estimate of the value of a vanadium redox flow battery for frequency regulation in texas," Applied Energy, vol. 113, pp. 189 - 198, 2014.

[16] G. He, Q. Chen, C. Kang, P. Pinson, and Q. Xia, "Optimal bidding strategy of battery storage in power markets considering performance-based regulation and battery cycle life," IEEE Trans. Smart Grid, vol. PP, no. 99, pp. 1-1, 2015.

[17] S. J. Lee, J. H. Kim, C. H. Kim, S. K. Kim, E. S. Kim, D. U. Kim, K. K. Mehmood, and S. U. Khan, "Coordinated control algorithm for distributed battery energy storage systems for mitigating voltage and frequency deviations," IEEE Trans. Smart Grid, vol. PP, no. 99, pp. 1-1, 2015.

[18] S. X. Chen, T. Zhang, H. B. Gooi, R. D. Masiello, and W. Katzenstein, "Penetration rate and effectiveness studies of aggregated bess for frequency regulation," IEEE Trans. Smart Grid, vol. PP, no. 99, pp. 1-1, 2015.

[19] H. Bevrani, Robust Power System Frequency Control. S Springer, 2009.

[20] C. E. Fosha and O. I. Elgerd, "The megawatt-frequency control problem: A new approach via optimal control theory," IEEE Trans. Power App. Syst.* (through 1985), vol. PAS-89, no. 4, pp. 563-577, April 1970.

[21] R. K. Cavin, M. C. Budge, and P. Rasmussen, "An optimal linear systems approach to load-frequency control," IEEE Trans. Power App. Syst.* (through 1985), vol. PAS-90, no. 6, pp. 2472-2482, Nov 1971.

[22] Y. Wang, R. Zhou, and C. Wen, "Robust load-frequency controller design for power systems," IEE Proceedings C - Generation, Transmission and Distribution, vol. 140, no. 1, pp. 11-16, Jan 1993.

[23] A. M. Stankovic, G. Tadmor, and T. A. Sakharuk, "On robust control analysis and design for load frequency regulation," IEEE Trans. Power Syst., vol. 13, no. 2, pp. 449-455, May 1998.

[24] S. Vachirasricirikul and I. Ngamroo, "Robust lfc in a smart grid with wind power penetration by coordinated v2g control and frequency controller," IEEE Trans. Smart Grid, vol. 5, no. 1, pp. 371-380, Jan 2014. 
[25] C. T. Pan and C. M. Liaw, "An adaptive controller for power system load-frequency control," IEEE Trans. Power Syst., vol. 4, no. 1, pp. 122-128, Feb 1989.

[26] R. R. Shoults and J. A. J. Ibarra, "Multi-area adaptive lfc developed for a comprehensive agc simulator," IEEE Trans. Power Syst., vol. 8, no. 2, pp. 541-547, May 1993.

[27] Y. Wang, R. Zhou, and C. Wen, "New robust adaptive load-frequency control with system parametric uncertainties," IEE Proceedings - Generation, Transmission and Distribution, vol. 141, no. 3, pp. 184-190, May 1994.

[28] C. Boonchuay, "Improving regulation service based on adaptive load frequency control in lmp energy market," IEEE Trans. Power Syst., vol. 29, no. 2, pp. 988989, March 2014.

[29] F. Beaufays, Y. Abdel-Magid, and B. Widrow, "Application of neural networks to load-frequency control in power systems," Neural Networks, vol. 7, no. 1, pp. 183 - 194, 1994. [Online]. Available: http://www.sciencedirect.com/science/article/ pii/0893608094900671

[30] L. R. Chang-Chien, Y. S. Wu, and J. S. Cheng, "Online estimation of system parameters for artificial intelligence applications to load frequency control," IET Generation, Transmission Distribution, vol. 5, no. 8, pp. 895-902, August 2011.

[31] H. A. Yousef, K. AL-Kharusi, M. H. Albadi, and N. Hosseinzadeh, "Load frequency control of a multi-area power system: An adaptive fuzzy logic approach," IEEE Trans. Power Syst., vol. 29, no. 4, pp. 1822-1830, July 2014.

[32] H. Bevrani and P. R. Daneshmand, "Fuzzy logic-based load-frequency control concerning high penetration of wind turbines," IEEE Systems Journal, vol. 6, no. 1, pp. 173-180, March 2012.

[33] Y. Mi, Y. Fu, C. Wang, and P. Wang, "Decentralized sliding mode load frequency control for multi-area power systems," IEEE Trans. Power Syst., vol. 28, no. 4, pp. 4301-4309, Nov 2013.

[34] A. M. Ersdal, L. Imsland, and K. Uhlen, "Model predictive load-frequency control," IEEE Trans. on Power Syst., vol. 31, no. 1, pp. 777-785, Jan 2016.

[35] J. Pahasa and I. Ngamroo, "Coordinated control of wind turbine blade pitch angle and phevs using mpcs for load frequency control of microgrid," IEEE Systems Journal, vol. 10, no. 1, pp. 97-105, March 2016.

[36] (2016) ERCOT:Day-Ahead Market Operating Procedure Manual. [Online]. Available: http://www.ercot.com/mktinfo/dam

[37] (2016) California ISO: Ancillary services. [Online]. Available: https://www.caiso. $\mathrm{com} /$ participate/Pages/MarketProducts/AncillaryServices/Default.aspx

[38] (2016) NYISO ancillary services manual. [Online]. Available: http://www.nyiso.com/public/webdocs/marketsoperations/documents/ ManualsandGuides/Manuals/Operations/ancserv.pdf 
[39] (2015) Energy storage market updates. [Online]. Available: http://www.cesa.org/ assets/Uploads/Webinar-Slides-9.30.15.pdf

[40] B. Xu, Y. Dvorkin, D. S. Kirschen, C. a. Silva-Monroy, and J.-P. Watson, "A comparison of policies on the participation of storage in u.s. frequency regulation markets," in IEEE PES Innovative Smart Grid Technologies Conference Europe, 2016, pp. 1-6.

[41] (2013) Faster Frequency Regulation Triples in PJM. [Online]. Available: http://www.greentechmedia.com/articles/read/ faster-frequency-regulation-triples-in-pjm

[42] T. Zhang, H. B. Gooi, S. X. Chen, and T. Goh, "Cost-effectiveness studies of the besss participating in frequency regulation," in 2015 IEEE ISGT ASIA, Nov 2015, pp. $1-6$.

[43] H. Chvez, R. Baldick, and J. Matevosyan, "Cps1 compliance-constrained agc gain determination for a single-balancing authority," IEEE Trans. Power Syst., vol. 29, no. 3, pp. 1481-1488, May 2014.

[44] N. Jaleeli and L. Vanslyck, "NERC's new control performance standards," IEEE Trans. Power Syst., vol. 14, no. 3, pp. 1092-1099, Aug. 1999.

[45] (2009) PJM Manual 12: Balancing Operations. [Online]. Available: www.pjm.com

[46] "IEEE Standard for Interconnecting Distributed Resources with Electric Power Systems," IEEE Std. 1547.2-2008, 2008.

[47] E. Rodrigues, A. Bizuayehu, and J. P. Catalao, "Analysis of requirements in insular grid codes for large-scale integration of renewable generation," in $T D$ Conference and Exposition, 2014 IEEE PES, April 2014, pp. 1-5.

[48] E. Troester, "New german grid codes for connecting pv systems to the medium voltage power grid."

[49] A. Molina-Garca, I. Muoz-Benavente, A. D. Hansen, and E. Gmez-Lzaro, "Demand-side contribution to primary frequency control with wind farm auxiliary control," IEEE Trans. Power Syst., vol. 29, no. 5, pp. 2391-2399, Sept 2014.

[50] "Grid integration of large-capacity renewable energy sources and use of largecapacity electrical energy storage," International Electrotechnical Commission, Tech. Rep., 2012.

[51] Z. S. Zhang, Y. Z. Sun, J. Lin, and G. J. Li, "Coordinated frequency regulation by doubly fed induction generator-based wind power plants," IET Renewable Power Generation, vol. 6, no. 1, pp. 38-47, January 2012.

[52] F. Diaz-Gonzalez, F. Bianchi, A. Sumper, and O. Gomis-Bellmunt, "Control of a flywheel energy storage system for power smoothing in wind power plants," IEEE Trans. Energy Convers., vol. 29, no. 1, pp. 204-214, Mar. 2014. 
[53] X. Li, D. Hui, and X. Lai, "Battery energy storage station (bess)-based smoothing control of photovoltaic (pv) and wind power generation fluctuations," IEEE Trans. Sust. Energy, vol. 4, no. 2, pp. 464-473, Apr. 2013.

[54] S. Teleke, M. Baran, S. Bhattacharya, and A. Huang, "Rule-based control of battery energy storage for dispatching intermittent renewable sources," IEEE Trans. Sustain. Energy, vol. 1, no. 3, pp. 117-124, Oct 2010.

[55] X. Feng, H. B. Gooi, and S. X. Chen, "Hybrid energy storage with multimode fuzzy power allocator for pv systems," IEEE Trans. Sustain. Energy, vol. 5, no. 2, pp. 389-397, Apr. 2014.

[56] A. J. Gaul, E. Handschin, W. Hoffmann, and C. Lehmkoster, "Establishing a rule base for a hybrid es/xps approach to load management," IEEE Trans. Power Syst., vol. 13, no. 1, pp. 86-93, Feb 1998.

[57] M. Datta and T. Senjyu, "Fuzzy control of distributed pv inverters/energy storage systems/electric vehicles for frequency regulation in a large power system," IEEE Trans. Smart Grid, vol. 4, no. 1, pp. 479-488, March 2013.

[58] D. Melo, G. H. Beng, and T. Massier, "Charging of electric vehicles and demand response management in a singaporean car park," in Power Engineering Conference (UPEC), 2014 49th International Universities, Sept 2014, pp. 1-6.

[59] P. Mercier, R. Cherkaoui, and A. Oudalov, "Optimizing a battery energy storage system for frequency control application in an isolated power system," IEEE Trans. Power Syst., vol. 24, no. 3, pp. 1469-1477, Aug 2009.

[60] A. Oudalov, D. Chartouni, and C. Ohler, "Optimizing a battery energy storage system for primary frequency control," IEEE Trans. Power Syst., vol. 22, no. 3, pp. 1259-1266, Aug 2007.

[61] G. Delille, B. Francois, and G. Malarange, "Dynamic frequency control support by energy storage to reduce the impact of wind and solar generation on isolated power system's inertia," IEEE Trans. Sustain. Energy, vol. 3, no. 4, pp. 931-939, Oct 2012 .

[62] F. D. Mohammadi, M. J. Ghorbani, A. Feliachi, and M. A. Choudhry, "Novel load frequency control approach based on virtual area error in a microgrid including pv and battery," in PES General Meeting - Conference Exposition, 2014 IEEE, July 2014 , pp. $1-5$.

[63] Y. Cheng, M. Tabrizi, M. Sahni, A. Povedano, and D. Nichols, "Dynamic available agc based approach for enhancing utility scale energy storage performance," IEEE Trans. Smart Grid, vol. 5, no. 2, pp. 1070-1078, March 2014.

[64] O. Palizban, K. Kauhaniemi, and J. M. Guerrero, "Evaluation of the hierarchical control of distributed energy storage systems in islanded microgrids based on std iec/iso 62264," in 2016 IEEE Power and Energy Society General Meeting (PESGM), July 2016, pp. 1-5. 
[65] M. Khalid and A. V. Savkin, "Model predictive control based efficient operation of battery energy storage system for primary frequency control," in Control Automation Robotics Vision (ICARCV), 2010 11th International Conference on, Dec 2010, pp. 2248-2252.

[66] X. Li, D. Hui, and X. Lai, "Battery energy storage station (bess)-based smoothing control of photovoltaic (pv) and wind power generation fluctuations," IEEE Trans. Sustain. Energy, vol. 4, no. 2, pp. 464-473, April 2013.

[67] I. Serban, R. Teodorescu, and C. Marinescu, "Energy storage systems impact on the short-term frequency stability of distributed autonomous microgrids, an analysis using aggregate models," IET Renewable Power Generation, vol. 7, no. 5, pp. 531-539, Sept 2013.

[68] J. Dang, J. Seuss, L. Suneja, and R. G. Harley, "Soc feedback control for wind and ess hybrid power system frequency regulation," IEEE Journal of Emerging and Selected Topics in Power Electronics, vol. 2, no. 1, pp. 79-86, March 2014.

[69] R. D. Masiello, B. Roberts, and T. Sloan, "Business models for deploying and operating energy storage and risk mitigation aspects," Proceedings of the IEEE, vol. 102, no. 7, pp. 1052-1064, July 2014.

[70] H. Amano, Y. Ohshiro, T. Kawakami, and T. Inoue, "Utilization of battery energy storage system for load frequency control toward large-scale renewable energy penetration," in Innovative Smart Grid Technologies (ISGT Europe), 2012 3rd IEEE PES International Conference and Exhibition on, Oct 2012, pp. 1-7.

[71] T. Masuta and A. Yokoyama, "Supplementary load frequency control by use of a number of both electric vehicles and heat pump water heaters," IEEE Trans. Smart Grid, vol. 3, no. 3, pp. 1253-1262, Sept 2012.

[72] C. A. Baone and C. L. DeMarco, "From each according to its ability: Distributed grid regulation with bandwidth and saturation limits in wind generation and battery storage," IEEE Trans. Control Syst. Technol., vol. 21, no. 2, pp. 384-394, March 2013.

[73] Z. Xu, R. Diao, S. Lu, J. Lian, and Y. Zhang, "Modeling of electric water heaters for demand response: A baseline pde model," IEEE Trans. Smart Grid, vol. 5, no. 5, pp. 2203-2210, Sept 2014.

[74] C. Ziras, E. Vrettos, and G. Andersson, "Primary frequency control with refrigerators under startup dynamics and lockout constraints," in 2015 IEEE Power Energy Society General Meeting, July 2015, pp. 1-5.

[75] Y. Qi, H. Jia, and Y. Mu, "Dynamic frequency control of autonomous microgrid based on family-friendly controllable loads," in Innovative Smart Grid Technologies (ISGT), 2013 IEEE PES, Feb 2013, pp. 1-6.

[76] Y. J. Kim, L. K. Norford, and J. L. Kirtley, "Modeling and analysis of a variable speed heat pump for frequency regulation through direct load control," IEEE Trans. on Power Syst., vol. 30, no. 1, pp. 397-408, Jan 2015. 
[77] H. Hao, Y. Lin, A. S. Kowli, P. Barooah, and S. Meyn, "Ancillary service to the grid through control of fans in commercial building hvac systems," IEEE Trans. Smart Grid, vol. 5, no. 4, pp. 2066-2074, July 2014.

[78] Y. Lin, P. Barooah, and J. L. Mathieu, "Ancillary services through demand scheduling and control of commercial buildings," IEEE Trans. on Power Syst., vol. PP, no. 99, pp. 1-1, 2016.

[79] F. Kennel, D. Gorges, and S. Liu, "Energy management for smart grids with electric vehicles based on hierarchical mpc," IEEE Trans. Ind. Informat., vol. 9, no. 3, pp. 1528-1537, Aug 2013.

[80] H. Huang and F. Li, "Sensitivity analysis of load-damping characteristic in power system frequency regulation," IEEE Trans. on Power Syst., vol. 28, no. 2, pp. 1324-1335, May 2013.

[81] S. A. Pourmousavi and M. H. Nehrir, "Introducing dynamic demand response in the lfc model," IEEE Trans. on Power Syst., vol. 29, no. 4, pp. 1562-1572, July 2014 .

[82] J. Xu, S. Liao, Y. Sun, X. Y. Ma, W. Gao, X. Li, J. Gu, J. Dong, and M. Zhou, "An isolated industrial power system driven by wind-coal power for aluminum productions: A case study of frequency control," IEEE Trans. Power Syst., vol. 30, no. 1, pp. 471-483, Jan 2015.

[83] H. Mohsenian-Rad, "Optimal bidding, scheduling, and deployment of battery systems in california day-ahead energy market," IEEE Trans. on Power Syst., vol. 31, no. 1, pp. 442-453, Jan 2016.

[84] T. Das, V. Krishnan, and J. D. McCalley, "High-fidelity dispatch model of storage technologies for production costing studies," IEEE Trans. Sustain. Energy, vol. 5, no. 4, pp. 1242-1252, Oct 2014.

[85] D. Pudjianto, C. Ramsay, and G. Strbac, "Virtual power plant and system integration of distributed energy resources," Renewable Power Generation, IET, vol. 1, no. 1, pp. 10-16, March 2007.

[86] A. Thavlov and H. W. Bindner, "Utilization of flexible demand in a virtual power plant set-up," IEEE Trans. Smart Grid, vol. 6, no. 2, pp. 640-647, March 2015.

[87] Y. Liu, H. Xin, Z. Wang, and D. Gan, "Control of virtual power plant in microgrids: a coordinated approach based on photovoltaic systems and controllable loads," IET Generation, Transmission Distribution, vol. 9, no. 10, pp. 921-928, 2015.

[88] M. Rahimiyan and L. Baringo, "Strategic bidding for a virtual power plant in the day-ahead and real-time markets: A price-taker robust optimization approach," IEEE Trans. Power Syst., vol. 31, no. 4, pp. 2676-2687, July 2016.

[89] Q. Zhao, Y. Shen, and M. Li, "Control and bidding strategy for virtual power plants with renewable generation and inelastic demand in electricity markets," IEEE Trans. Sustain. Energy, vol. 7, no. 2, pp. 562-575, April 2016. 
[90] E. G. Kardakos, C. K. Simoglou, and A. G. Bakirtzis, "Optimal offering strategy of a virtual power plant: A stochastic bi-level approach," IEEE Trans. Smart Grid, vol. 7, no. 2, pp. 794-806, March 2016.

[91] P. Moutis and N. D. Hatziargyriou, "Decision trees-aided active power reduction of a virtual power plant for power system over-frequency mitigation," IEEE Trans. Ind. Informat., vol. 11, no. 1, pp. 251-261, Feb 2015.

[92] M. K. Petersen, L. H. Hansen, J. Bendtsen, K. Edlund, and J. Stoustrup, "Heuristic optimization for the discrete virtual power plant dispatch problem," IEEE Trans. Smart Grid, vol. 5, no. 6, pp. 2910-2918, Nov 2014.

[93] E. Mashhour and S. Moghaddas-Tafreshi, "Bidding strategy of virtual power plant for participating in energy and spinning reserve markets part I: Problem formulation," IEEE Trans. Power Syst., vol. 26, no. 2, pp. 949-956, May 2011.

[94] J. M. Maciejowski, Predictive Control with Constraints. Prentice Hall, 2002.

[95] M. D. Galus, S. Koch, and G. Andersson, "Provision of load frequency control by phevs, controllable loads, and a cogeneration unit," IEEE Trans. Ind. Electron., vol. 58, no. 10, pp. 4568-4582, Oct 2011.

[96] H. Amano, Y. Ohshiro, T. Kawakami, and T. Inoue, "Utilization of battery energy storage system for load frequency control toward large-scale renewable energy penetration," in IEEE PES Int. Conf. and Exhibition on Innovative Smart Grid Technologies (Europe), Oct 2012, pp. 1-7.

[97] H. Tazvinga, X. Xia, and J. Zhang, "Minimum cost solution of photovoltaic-dieselbattery hybrid power systems for remote consumers," Solar Energy, vol. 96, no. 0, pp. 292-299, 2013.

[98] M. Shiroei, M. R. Toulabi, and A. M. Ranjbar, "Robust multivariable predictive based load frequency control considering generation rate constraint," Int. Journal of Electrical Power $\& 3$ Energy Systems, vol. 46, no. 0, pp. 405-413, 2013.

[99] N. Atic, D. Rerkpreedapong, A. Hasanovi, and A. Feliachi, "Nerc compliant decentralized load frequency control design using model predictive control," in IEEE Power \& Engineering Society General Meeting, vol. 2, July 2003, pp. 554-559.

[100] M. Khalid and A. Savkin, "Model predictive control based efficient operation of battery energy storage system for primary frequency control," in 11th Int. Conf. on Control Automation Robotics Vision, Dec 2010, pp. 2248-2252.

[101] R. Scattolini, "Architectures for distributed and hierarchical model predictive control: A review," Journal of Process Control, vol. 19, no. 5, pp. 723-731, 2009.

[102] A. H. G. Lez, J. L. Marchetti, and D. Odloak, "Robust model predictive control with zone control," IET Control Theory Applications, vol. 3, no. 1, pp. 121-135, January 2009.

[103] S. X. Chen, H. B. Gooi, and M. Q. Wang, "Sizing of energy storage for microgrids," IEEE Trans. Smart Grid, vol. 3, no. 1, pp. 142-151, March 2012. 
[104] M. Giuntoli and D. Poli, "Optimized thermal and electrical scheduling of a large scale virtual power plant in the presence of energy storages," IEEE Trans. Smart Grid, vol. 4, no. 2, pp. 942-955, Jun. 2013.

[105] European Virtual Fuel Cell Power Plant. (2014) Management Summary. [Online]. Available: http://ec.europa.eu/energy/efficiency/industry/doc/euvpp.pdf

[106] FENIX. (2014) Fenix Projet Summary. [Online]. Available: http://www. fenix-project.org/

[107] P. Kundur, Power system stability and control. McGraw-hill, 1993.

[108] S. X. Chen, H. B. Gooi, N. Xia, and M. Q. Wang, "Modelling of lithium-ion battery for online energy management systems," IET Electr. Syst. Transp., vol. 2, no. 4, pp. 202-210, Dec. 2012.

[109] M. L. Lazarewicz and T. Ryan, "Grid-scale frequency regulation using flywheels," Beacon Power Corporation, Tech. Rep., 2010.

[110] B. Washom, J. Dilliot, D. Weil, J. Kleissl, N. Balac, W. Torre, and C. Richter, "Ivory tower of power: Microgrid implementation at the university of california, san diego," Power and Energy Magazine, IEEE, vol. 11, no. 4, pp. 28-32, July 2013.

[111] S. J. Chiang, K. T. Chang, and C. Y. Yen, "Residential photovoltaic energy storage system," IEEE Trans. Ind. Electron., vol. 45, no. 3, pp. 385-394, Jun 1998.

[112] M. Gallieri and J. M. Maciejowski, "Lasso mpc: Smart regulation of over-actuated systems," in American Control Conference (ACC), June 2012, pp. 1217-1222.

[113] K. Worthmann, C. M. Kellett, P. Braun, L. Grne, and S. R. Weller, "Distributed and decentralized control of residential energy systems incorporating battery storage," IEEE Trans. Smart Grid, vol. 6, no. 4, pp. 1914-1923, July 2015.

[114] A. Ulbig, M. Arnold, S. Chatzivasileiadis, and G. Andersson, "Framework for multiple timescale cascaded MPC application in power systems," in 18th IFAC World Congr., 2011, 2011, pp. $10472-10480$.

[115] A. Hooshmand, B. Asghari, and R. K. Sharma, IEEE Trans. Sustain. Energy, no. 4 , Oct.

[116] H. H. Abdeltawab and Y. A. I. Mohamed, "Market-oriented energy management of a hybrid wind-battery energy storage system via model predictive control with constraint optimizer," IEEE Trans. Ind. Electron., vol. 62, no. 11, pp. 6658-6670, Nov 2015.

[117] Energy market company: Guide to prices. [Online]. Available: https: //www.emcsg.com/MarketData/GuideToPrices

[118] Z. O. LLC, "Ziena optimization experts in nonlinear optimization," http://www.ziena.com/knitro.htm, Apr. 2013. 
[119] (2015) Matlab and GAMS: Interfacing Optimization and Visualization Software via the GDXMRW Utilities. [Online]. Available: http://www.gams.com/ $\sim_{\text {steve/ }}$ gdxmrw.html

[120] S. X. Chen, T. Zhang, H. B. Gooi, R. D. Masiello, and W. Katzenstein, "Penetration rate and effectiveness studies of aggregated bess for frequency regulation," IEEE Trans. Smart Grid, vol. 7, no. 1, pp. 167-177, Jan 2016.

[121] (2014) PJM Ancillary Services. [Online]. Available: http://www.pjm.com/ markets-and-operations/ancillary-services.aspx

[122] A. U. Haque, M. H. Nehrir, and P. Mandal, "A hybrid intelligent model for deterministic and quantile regression approach for probabilistic wind power forecasting," IEEE Trans. on Power Syst., vol. 29, no. 4, pp. 1663-1672, July 2014.

[123] T. Niknam, R. Azizipanah-Abarghooee, and M. R. Narimani, "An efficient scenario-based stochastic programming framework for multi-objective optimal micro-grid operation," Applied Energy, vol. 99, pp. 455 - 470, 2012. [Online]. Available: http://www.sciencedirect.com/science/article/pii/S0306261912003030

[124] (2009) PJM Manual 28: Operating Agreement Accounting. [Online]. Available: http://www.pjm.com/ /media/documents/manuals/m28.ashx 


\section{VITA}

Zhang Tian was born in 1990 in Chongqing, P. R. China. She received her B.S. degree in Electrical Engineering and its Automation from Tianjin University, Tianjin, China. She is currently working as a project officer in Energy Research Institute @ NTU. Her research interests include smart energy management systems, virtual power plants, renewable energy sources and energy storage systems.

This research work has led to the following publications.

\section{i. Journal papers:}

[1] T. Zhang, S. X. Chen, H. B. Gooi and J. M. Maciejowski, " A Hierarchical EMS for Aggregated BESSs in Energy and Performance-Based Regulation Markets," in IEEE Transactions on Power Systems, vol. 32, no. 3, pp. 1751-1760, May 2017.

[2] S. Chen, T. Zhang, H. B. Gooi, R. D. Masiello and W. Katzenstein, "Penetration Rate and Effectiveness Studies of Aggregated BESS for Frequency Regulation," inIEEE Transactions on Smart Grid, vol. 7, no. 1, pp. 167-177, Jan 2016.

\section{ii. Conference papers:}

[1] T. Zhang and H. B. Gooi, "Hierarchical MPC-based energy management and frequency regulation participation of a virtual power plant," IEEE PES Innovative Smart Grid Technologies, Europe, Istanbul, 2014, pp. 1-5.

[2] T. Zhang, H. Beng Gooi, S. Chen and T. Goh, "Cost-effectiveness studies of the BESSs participating in frequency regulation,"2015 IEEE Innovative Smart Grid Technologies - Asia (ISGT ASIA), Bangkok, 2015, pp. 1-6. 